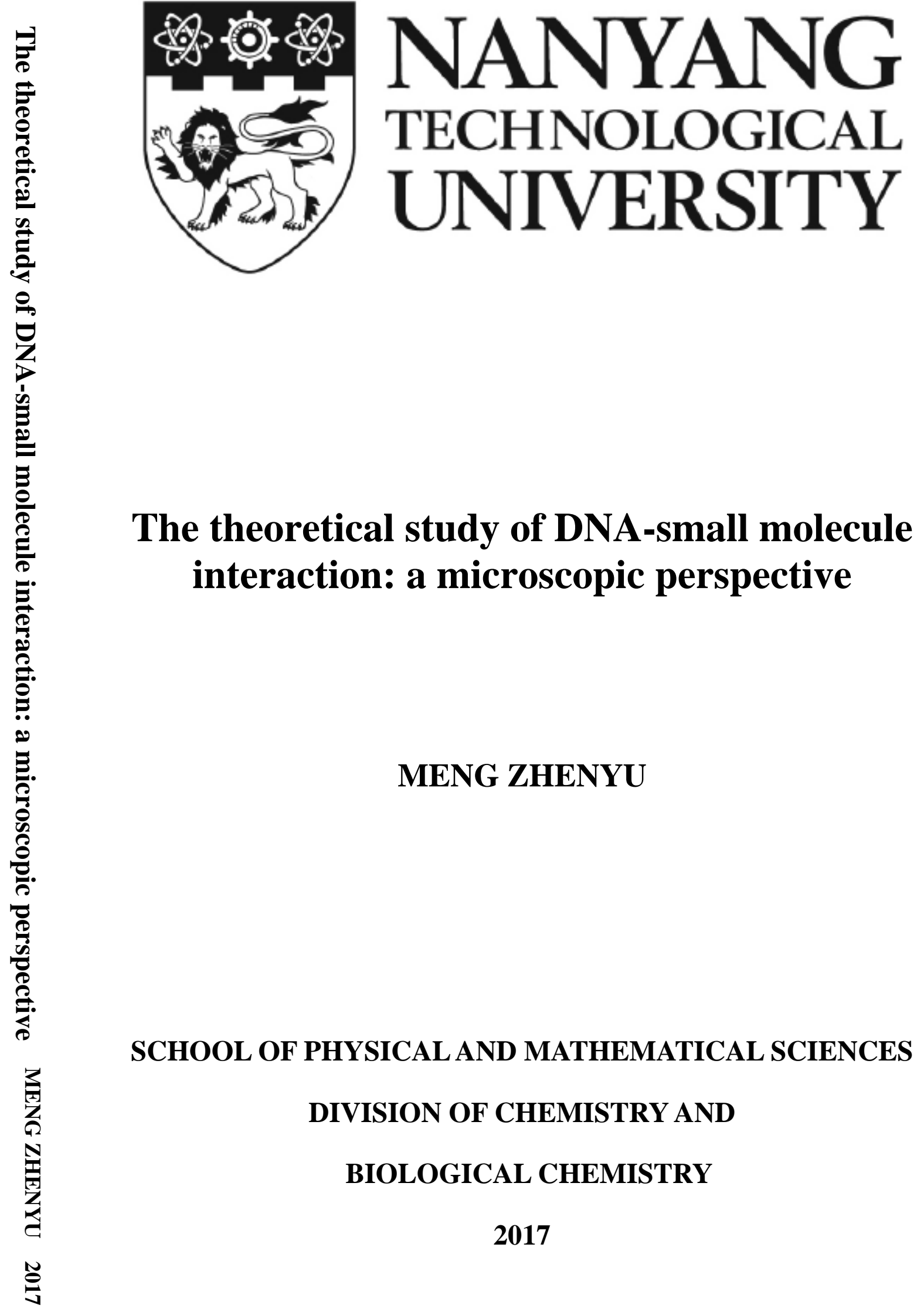




\title{
The theoretical study of DNA-small molecule interaction: a microscopic perspective
}

\author{
MENG ZHENYU
}

SCHOOL OF PHYSICAL AND MATHEMATICAL SCIENCES

DIVISION OF CHEMISTRY AND

BIOLOGICAL CHEMISTRY

A thesis submitted to the Nanyang Technological University in partial fulfillment of the requirement for the degree of Doctor of Philosophy 


\section{Acknowledgement}

The four-year phD study will come to an end. During this period, there has been happinesses, sorrows, enjoyments after success, and frustrations after failures. But when I finished the last sentence of the thesis, I knew all was worthy.

First of all, I would like to extend my sincere gratitude to Dr. Shao Fangwei, who provided me such a wonderful opportunity to pursue the heights of science. More importantly, she encouraged me to convert my project onto theoretical field when I suffered helpless difficulties in my bench work. Her instruction and advice would be an invaluable treasure for my future academic life.

Secondly my thanks would go to Dr. Mu Yuguang who guided me to step into the gate of computational chemistry. His patient instruction and inspired advice helped me a lot for my research. I also would like to thank Dr. Hajime Hirao as a partial supervisor for the last two years of my phD study. His strict demands and attitude of excellence improved my self-discipline greatly.

Many thanks to the current and previous group members: Dr Wang Jintao, Dr. $\mathrm{Xu}$ Baochang, Dr. Xuan Shuguang, Dr. Wu qiong, Dr. Wong Jiun-ru, Dr. Devi Ghitali, Dr Hu Lianzhe, Dr Wu Jun, Dr Xu Dechen, Dr. Chen Xiang, Dr. Wang Liying, Dr. Kenny Yong, Lu Kaihui, Wu Jingyuan, He Lei, Tian Keyin, Guo Juanjuan. Thank you guys for always being friendly and helpful, which makes the four-year hard work full of happiness and memories. I also would like to thank the members from Dr. Mu's group: Dr. Luo Di, Dr. Ning Lulu, Dr. Yaw Awuni, Amr Ali Mokhtar Alhossary, Zheng 
Liangzhen, Liu Yang, Justin Ng; and the members from Dr. Hirao's group: Dr. Mikhail Ozhgibesov, Dr. Sareeya Bureekaew, Dr. Kazuki Doitomi, Dr. Wilson Ng, Dr. Chuanprasit Pratanphorn, Dr. Li Xu, Dr. Wang Xiaoqing, Xu Kai, Adhitya Mangala Putra Moeljadi, Wang Ruixing.

And my friends, Wang Jin, Yang Yijie, Liu Yiran, Hu Ming, Yang Zhe, Wang Hong, thanks for your support and help. There're so many people I haven't mentioned in this acknowledgement, I would like to express my sincere thanks to all of you.

Special thanks would go to Dr. Thomas Kubar who provided me the code for DFTB calculation; his kind instruction is highly appreciated.

I wish to acknowledge NTU to provide me the research scholarship as my financial support.

Last but not least, I would like to express my gratitude to my parents. Thank you for your continued encouragement and care.

The help from all my beloved reminds me to endeavor for a higher peak. As Einstein put it, "A hundred times a day I remind myself that my inner and outer life depend on the labors of other men, living and dead, and that I must exert myself in order to give in the measure as I have received and am still receiving." I will not let you down.

Thanks again for all of you. 


\section{Abstract}

The microscopic studies of DNA always involve the interaction between DNA and small molecules. Previously, much work has been done in our group with regard to the projects, such as DNA charge transport (CT) in ionic liquid, and the interaction between G-quadruplex and metal complex. Such researches provide much inspiration and perspective to tune the dynamic conformation of the secondary structure of DNA and thus adjust the properties of DNA upon the binding of small molecules. However, it seems necessary to explain such experimental results with the microscopic structural processes. Since experimental characterization of DNA conformation, especially with dynamic motion of nucleobase pairs, were extremely limited, theoretical and computational approaches would be a desired exploration to better understand the experimental data. With such a goal in mind, series computational studies have been done in this thesis.

Chapter 1 will review the current available computational methods to study the dynamic structures and interactions between small molecules and DNA on different spatial scales. The basic principles and algorithms will be reviewed firstly, followed by the examples of advances in DNA simulation which explored the different structural properties of DNA under various situations.

In chapter 2, molecular dynamics accompanied by Principal Component Analysis (PCA) will be used to analysis the subtle conformational change of duplex DNA in water and hydrated ionic liquid. The results demonstrated the confining effects of 
ionic liquid to DNA conformation, and further comparison of internal parameters showed that the most vulnerable region of DNA resided in the G-C/A-T neighbouring step, to which the cations showed binding affinity at the same time.

Further QM/MM calculation in the following chapter 3 illustrated that ionic liquids can facilitate the charge transport (CT) rate by increasing the coupling between adjacent nucleobase pairs. The rate was estimated under flickering resonance model with the required parameters computed by DFTB (Density functional theory tight-binding) method. Such theoretical research confirmed our previous experimental results and can provide more insights into the relationship between CT rate and dynamic conformation of duplex DNA upon binding of solvent molecules.

Varieties of metal complexes as G-quadruplex binder will be introduced in chapter 4. The planar Pt(II) complexes with different side chains was first explored. By docking simulation, the stabilization effect of side chain was illustrated. Next, the theoretical research on octahedral $\mathrm{Ru}$ (II) complex confirmed the importance of $\mathrm{z}$-axis ligands which located at the ion channel to stabilize G-quartet. Further the customized force field was built for a binuclear Pt complex and molecular dynamics was employed to explore the possible structure after cross-linking reaction between binuclear complex and G-quadruplex.

In appendix an experimental work about direct RNA-DNA FISH (fluorescent in-situ hybridization) was introduced. Using amino-labeled oligonucleotide probes, a simple, robust and low-noise method for simultaneous detection of RNA and DNA by 
fluorescence in situ hybridization was established. With this modified probes, we demonstrated that the method can be applied to study a wide range of RNA and DNA targets at the single-cell and single molecule level in cellular contexts. 


\section{Content}

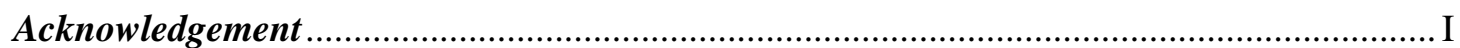

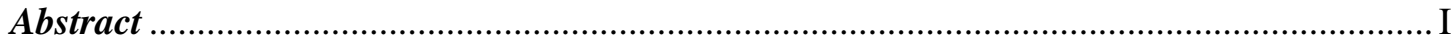

Chapter 1: The current theoretical and computational methods to study DNA structure

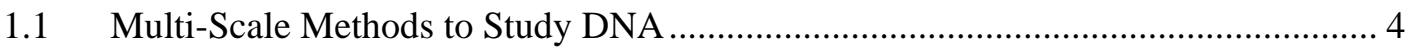

1.1.1 Molecular dynamics (MD) simulation............................................................. 4

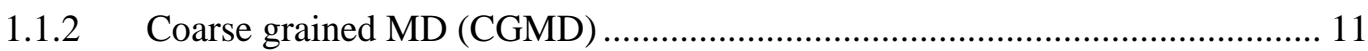

1.1.3 Replica-exchange molecular dynamics (REMD) .......................................... 11

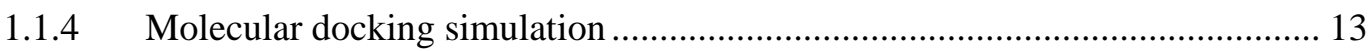

1.1.5 Quantum mechanics (QM) method ........................................................... 14

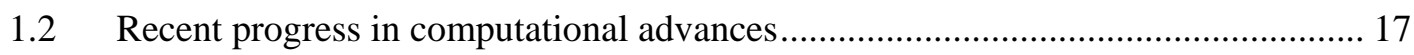

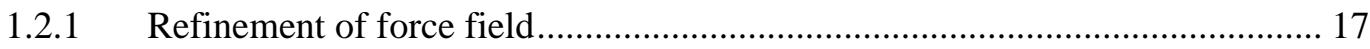

1.2.2 The external environmental impact on DNA structures .................................. 18

1.2.3 The simulation of non-canonical DNA structures ......................................... 18

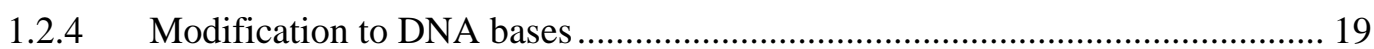

1.2.5 DNA interacting with binding molecules …............................................... 19

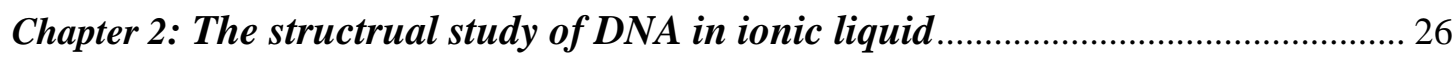

2.1 Motivation: The study of DNA conformational change in IL ................................ 27

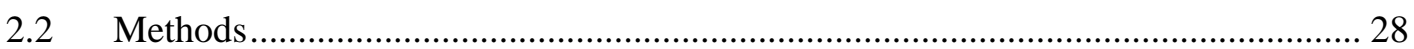

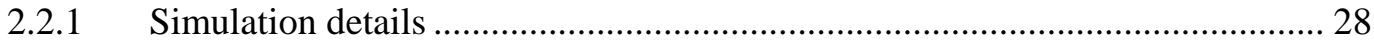

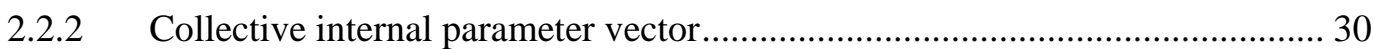

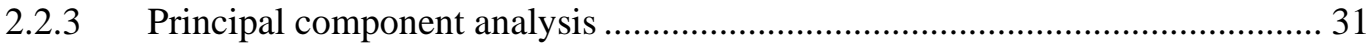

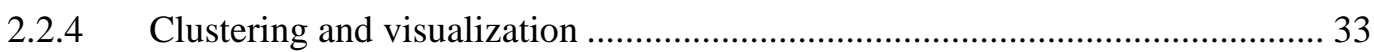

2.2.5 Contact number of cations towards minor groove base.................................... 33

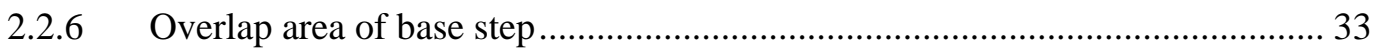

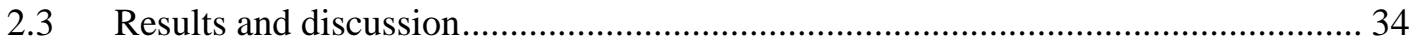

2.3.1 The subtle conformational change in IL ............................................................ 34

2.3.2 C-G and its proximal region showed more conformational change ................. 37

2.3.3 Binding of cations to minor groove induced conformational change ............... 41 
2.3.4 Dynamic motion of DNA induced by the change of binding mode

2.4 Conclusion 50

Chapter 3: A Theoretical Study of DNA-Mediated Charge Transport in Ionic Liquid

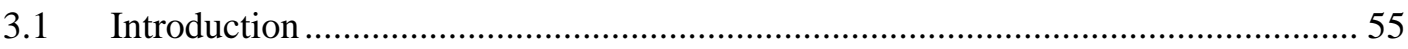

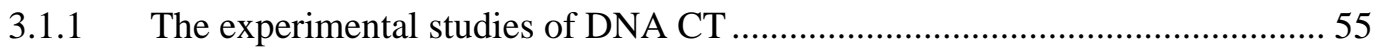

3.1.2 CT through DNA stacked base pairs .............................................................. 55

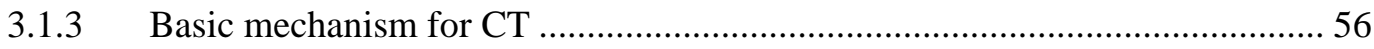

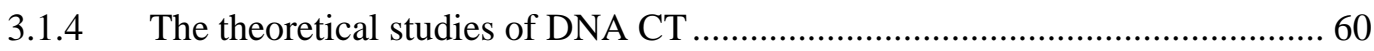

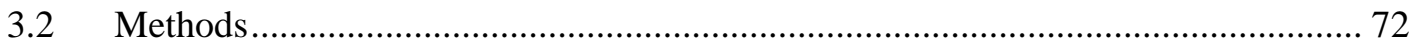

3.2.1 QM calculation employing fragment orbital density functional theory

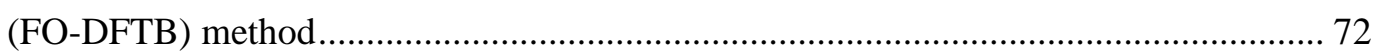

3.2.2 Calculation of CT rate using different multistate model ................................ 74

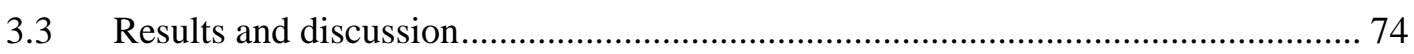

3.3.1 IL stabilized onsite energy of DNA base ....................................................... 74

3.3.2 IL can facilitate CT rate under FR model ..................................................... 76

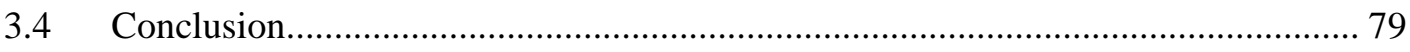

Chapter 4: Theoretical investigation of novel metal complex as G-quadruplex binder

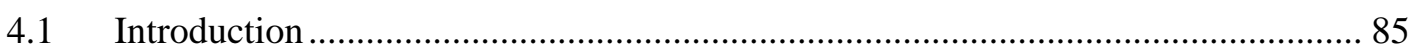

4.1.1 The structure and function of G-quadruplex ................................................. 85

4.1.2 Metal complex as G-quadruplex stabilizer .................................................. 88

4.1.3 Direct coordination of the metal to G-quadrulex .............................................. 90

4.1.4 The theoretical methods to explore G-quadruplex stabilizer............................ 92

4.2 Aza-bridged bisphenanthrolinyl Pt(II) complexes: efficient stabilization and topological selectivity on telomeric G-quadruplexes ......................................................... 93

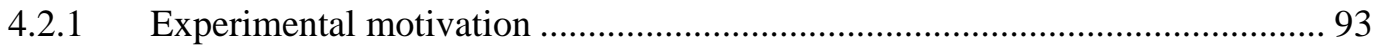

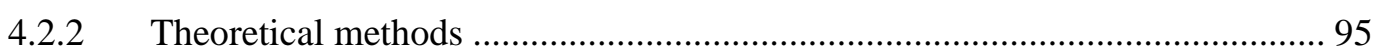

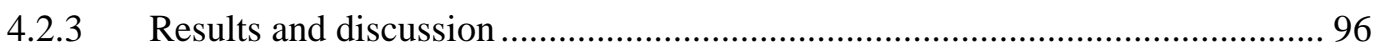

4.3 Octahedral ruthenium complexes selectively stabilize G-quadruplexes .................. 99

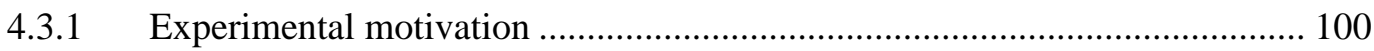


4.4 Dinuclear platinum compounds specially cross-link to parallel human c-myc

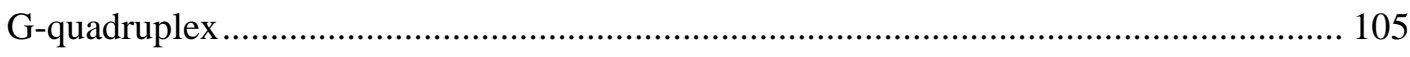

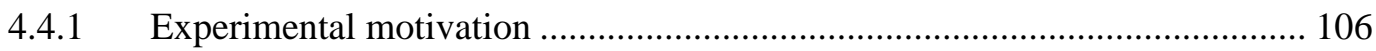

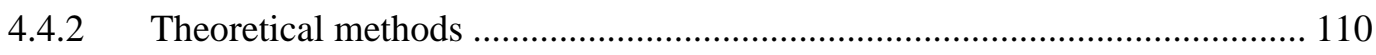

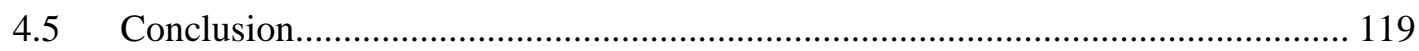

\section{Appendix: Using Amino-Labeled Nucleotide Probes for Simultaneous Single}

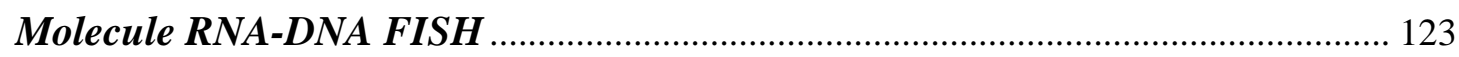

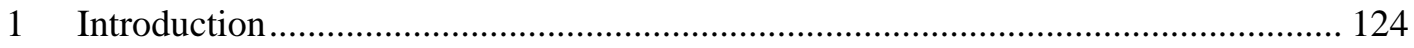

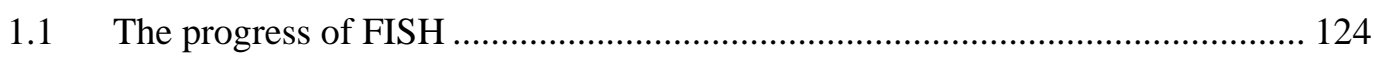

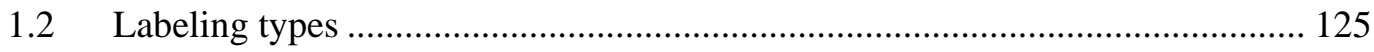

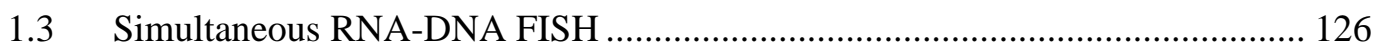

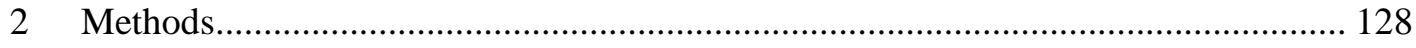

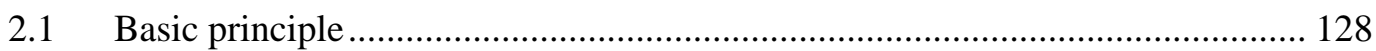

2.2 Preparetion of amino-labeled oligonucleotide probes ....................................... 129

2.3 Electrophoretic mobility shift assay ............................................................ 130

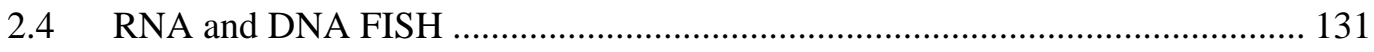

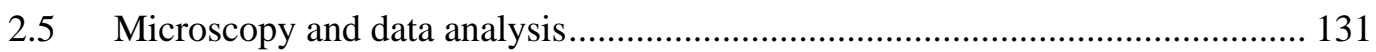

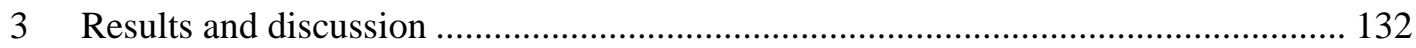

3.1 Characterization of synthetic amino-oligonucleotide...................................... 132

3.2 Simultaneous detection of Terra and telomere DNA …................................... 134

3.3 Simultaneous detection of single mRNA molecule with DNA ......................... 135

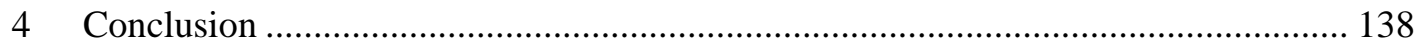




\section{Chapter 1}

The current theoretical and computational methods to study DNA structure 
DNA, short for deoxynucleic acid, plays an important role in heredity of species. As a biological polymer, DNA heavily wrapped up itself to form chromosomes with the help of protein. Within the chromosome, part of DNA sequence is the template recording genetic information (gene). After transcription and translation, these sequences can be decoded into thousands kinds of proteins which play different biological functions. Because of its vital importance in life processes, scientists began to study its structure, biological function and physical/chemical property long time ago. From Mendel to Morgan, many pioneering work has been done with regards to genetics. But it was until 1950s that Hershey and Case proved that DNA was the main genetic material ${ }^{1}$. In 1953 Watson and Crick discovered the double helix structure of $\mathrm{DNA}^{2}$. Such a milestone experiment opened the door to the microscopic study of DNA structure. Nowadays, the researches on relationship between DNA and genetics, such as epigenetics, DNA sequencing and DNA repairing, are still popular topics. In the meanwhile, some other fields focusing on the DNA itself are also on their way. Based on its well-behaved biocompatibility, DNA is an ideal media in the application for drug delivery ${ }^{3,4}$, cell imaging ${ }^{5}$ et al. Moreover, the unique complementary pairing property of DNA provides scientists such a novel way to handle DNA as a scaffold ${ }^{6,7}$. The DNA origami ${ }^{8,9}$ can form varieties of complex and fantastic structures, and DNA-based logic gates ${ }^{10}$ explore the possibilities to use DNA as a unit to build biochemical circuits.

With the progress of experimental work on DNA, people may meet some bottleneck with regard to the limitation of instrument which restrains them from the 
more microscopic observation. To solve such problems, many scientists tried to find some in silico methods as an estimation as well as confirmation to experimental studies. Such trials have achieved many successes ${ }^{11}$. Up to now, molecular dynamics (MD) simulation is already a mature method to illustrate many types of biological processes, such as the DNA melting temperature ${ }^{12}$, the binding of ligand to different structures of $\mathrm{DNA}^{13}$ and change of DNA conformations ${ }^{14}$. Besides, quantum mechanics (QM) methods also see their advantages when dealing with more microscopic properties of DNA, such as on-site energies ${ }^{15}$. The collaboration of experimental and computational methods has been proved to be a powerful way for study of microscopic properties of DNA and DNA-related studies.

As shown in figure 1-1, the simulation of DNA spans wide range of size scale as well as the time scale, according to the degree of refinement, the method of simulation goes from Ab-initio QM method to coarse grained MD. This chapter will first briefly introduce the theoretical background of these simulation methods; later the important application of these methods towards different aspects of DNA properties will be mentioned. 


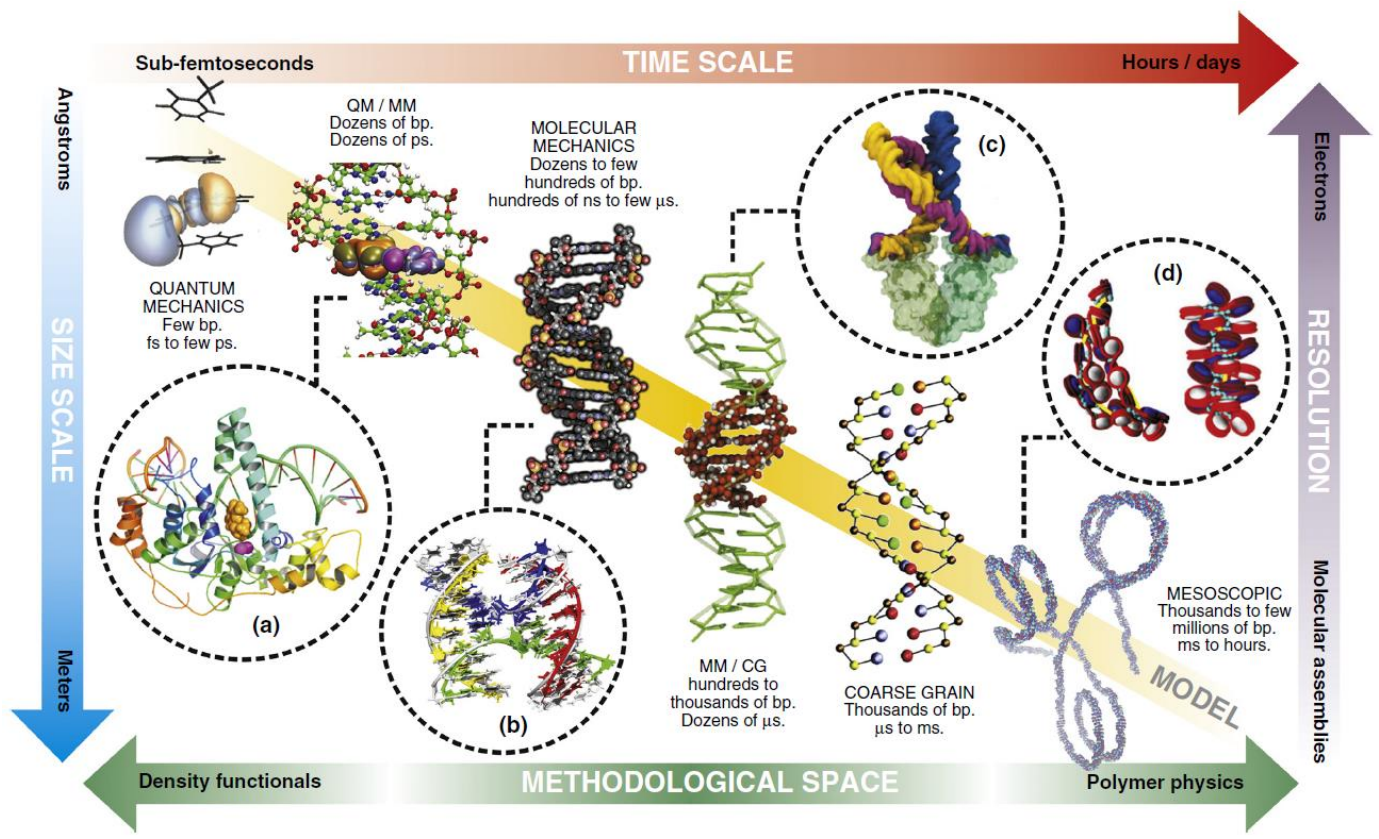

Figure 1-1 The multi-scale DNA simulation. The graph was taken from ref 16

\subsection{Multi-Scale Methods to Study DNA}

\subsubsection{Molecular dynamics (MD) simulation}

The moderate computational cost and significant accuracy makes MD the most widely-used theoretical method to study not only DNA but also other kinds of bio-molecules such as protein and lipids. The application of MD appeared in very different fields of theoretical DNA research, which will be introduced thoroughly in section 1.2. Here the principle and algorithms used in MD will be firstly reviewed.

\subsubsection{Basic principle}

Full atomic molecular dynamics is a widely used computational method based on classical Newton mechanics. With position $r_{i}(t)$ and velocity $v_{i}(t)$ for each atom known, the potential energy, $\mathrm{E}(\mathrm{t})$, can be calculated according to certain force field. Thereafter the force, $F_{i}(t)$, can be calculated: 


$$
F_{i}(t)=-\frac{\partial E(t)}{\partial r_{i}(t)}
$$

And the position and velocity at next time step, $r_{i}(t+\Delta t)$ and $v_{i}(t+\Delta t)$, can be integrated. The usually used integration method is leap-frog method ${ }^{17}$ :

$$
\begin{gathered}
r_{i}(t+\Delta t)=r_{i}(t)+\Delta t v_{i}(t+\Delta t / 2) \\
v_{i}(t+\Delta t)=v_{i}(t-\Delta t / 2)+\Delta t \frac{F_{i}(t)}{m_{i}}
\end{gathered}
$$

with

$$
\begin{aligned}
& v_{i}(t-\Delta t / 2) \equiv \frac{r_{i}(t)-r_{i}(t-\Delta t)}{\Delta t} \\
& v_{i}(t+\Delta t / 2) \equiv \frac{r_{i}(t+\Delta t)-r_{i}(t)}{\Delta t}
\end{aligned}
$$

The term $\mathrm{v}_{\mathrm{i}}(\mathrm{t}-\Delta \mathrm{t} / 2)$ and $\mathrm{v}_{\mathrm{i}}(\mathrm{t}+\Delta \mathrm{t} / 2)$ will be calculated before integration step. The following is the common work flow for MD simulation:

1) Reading in the simulation parameters, such as time step, total steps, temperature, pressure, positions of atoms et al.

2) Initializing the velocities according to the set temperature.

3) Computing the forces for each particle.

4) Updating the position and velocity according to integration algorithm. This step is repeated until the simulation reaches the desired time length.

5) Outputing the final position and velocity

The potential and kinetic energy can be computed for each time step. Using MD simulation the time evolution of the system can be plotted and useful information, 
such as the potential energy, the number of hydrogen bonding and the fluctuation of atom group can be easily computed.

\subsubsection{Force field}

The force field in MD refers to the formula and the parameters therein to calculate the potential energy of the system. For the biomolecule simulation, there are several different force fields. The widely used AMBER force field Hamiltonian can be written as ${ }^{18}$ :

$$
\begin{gathered}
E_{\text {total }}=\sum_{\text {bonds }} k_{b}\left(r-r_{0}\right)^{2}+\sum_{\text {angles }} k_{\theta}\left(\theta-\theta_{0}\right)^{2}+ \\
\sum_{\text {dihedrals }} V_{n}[1+\cos (n \phi-\gamma)]+\sum_{i=1}^{N-1} \sum_{j=i+1}^{N}\left[\frac{A_{i j}}{R_{i j}^{12}}-\frac{B_{i j}}{R_{i j}^{6}}+\frac{q_{i} q_{j}}{\varepsilon R_{i j}}\right] \quad \text { Eq } 1.6
\end{gathered}
$$

In which the $\mathrm{k}_{\mathrm{b}}$ is the force constant for each bond and $\mathrm{r}_{0}$ the equilibrium bond length; the $\mathrm{k}_{\theta}$ is the force constant for each angle and $\theta_{0}$ the equilibrium angle value; the $V_{n}$ is the torsional barrier, $\gamma$ the phase and $n$ the periodicity; $A_{i j}$ and $\mathrm{B}_{\mathrm{ij}}$ are the parameters for Leonard-Jones potential. All these above-mentioned parameters differ in value for different version of AMBER force field. The atomic charge $q_{i}$ is usually computed beforehand using RESP or some other methods. When conducting MD simulation, each bond $\mathrm{r}$, angle $\theta$, dihedral $\phi$, and nonbonding distance $R_{i j}$ can be calculated according to the position of atom. 


\subsubsection{Common algorithms for MD simulation}

\subsection{Periodic box condition (PBC)}

Biomolecule simulation always deals with an ensemble instead of a single molecule. However, to simulate that many molecules at the same time seems impossible and unnecessary. Therefore, PBC was introduced to save the computational resource and mimic a real condensed environment. The basic idea is to simulate a separate box of atoms, while in the simulation, a given particle is assumed to interact with all the particles inside the box as well as all the particles in the same periodic cells; in the meawhile we put the atom out of box size back to the box. The idea is illustrated in Figure 1-2.

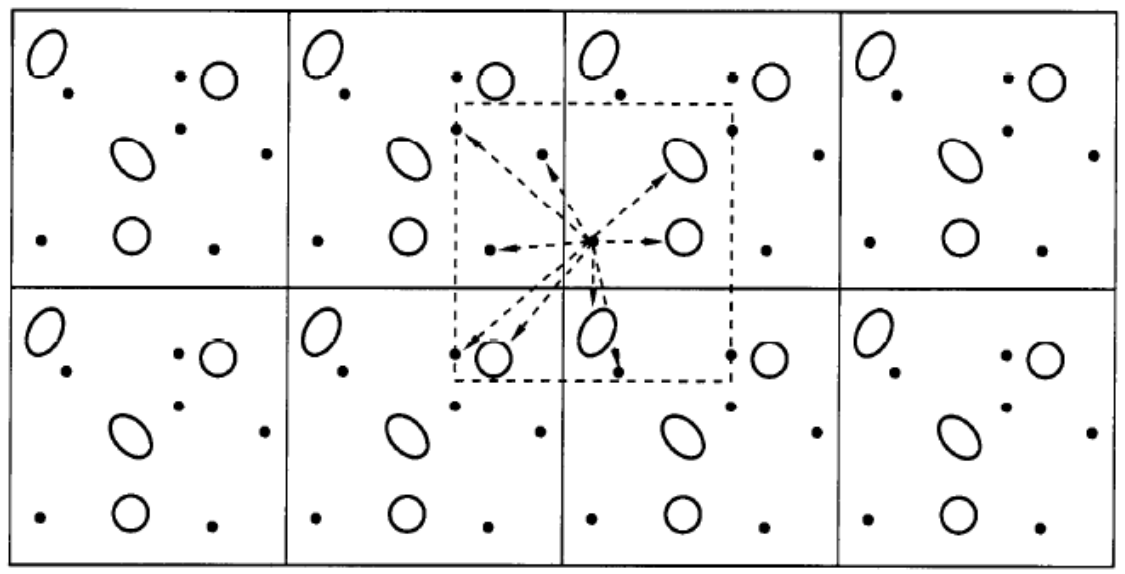

Figure 1-2 The scheme of periodic boundary condition. The graph was taken from ref 17 


\subsection{Particle meshed Ewald (PME)}

When we calculate electrostatic interaction using normal Coulomb Law in periodic boundary condition, the results seldom converge. To overcome such difficulty, a Gaussian distribution of the same amount of opposite charge was introduced to each point charge. At the same time a compensating charge with the same Gaussian distribution was also added to remain the total charge unchanged. The potential energy from point charge together with the opposite Gaussian charge is short ranged in real space ${ }^{17}$ :

$$
E^{S}=\frac{1}{4 \pi \varepsilon_{0}} \frac{1}{2} \sum_{\boldsymbol{n}} \sum_{i=1}^{N} \sum_{j=1}^{N}{ }^{\prime} \frac{q_{i} q_{j}}{\left|r_{i}-r_{j}+\boldsymbol{n} L\right|} \operatorname{erfc}\left(\frac{\left|r_{i}-r_{j}+\boldsymbol{n} L\right|}{\sqrt{2} \sigma}\right)
$$

in which $\boldsymbol{n}$ is the periodic vector and $\sigma$ is the standard deviation of Gaussian distribution. The contribution from compensating Gaussian charge is also converged in Fourier space:

$$
\begin{gathered}
E^{L}=\frac{1}{2 V \varepsilon_{0}} \sum_{\boldsymbol{k} \neq 0} \frac{e^{-\sigma^{2} k^{2} / 2}}{k^{2}}|S(\boldsymbol{k})|^{2} \\
S(\boldsymbol{k}) \equiv \sum_{i=1}^{N} q_{i} e^{i \boldsymbol{k} \cdot \boldsymbol{r}_{i}}
\end{gathered}
$$

in which $\boldsymbol{k}$ is a reciprocal vector. At last we need to correct the $E^{L}$ to avoid self-interaction:

$$
E^{\text {self }}=\frac{1}{4 \pi \varepsilon_{0}} \frac{1}{\sqrt{2 \pi} \sigma} \sum_{i=1}^{N} q_{i}^{2}
$$

The final electronic potential energy is the sum of all three above mentioned terms. Such method is called Ewald summation. When we deal with large amount of 
particles, it is still time-consuming using original Ewald summation. A further acceleration method was introduced in which all the point charges are attributed to mesh points using a spline function (PME) $)^{19}$.

\subsection{Thermostat and barostat}

To simulate a "real" system, people need to maintain the temperature and internal pressure of the system. Many methods to fulfill this goal exist. The Berendsen Thermostat $^{20}$ is commonly used, the idea is to multiply the atom velocity with a scale factor $\alpha$ :

$$
\alpha=\left(1+\frac{\Delta t}{\tau_{T}}\left(\frac{T}{T(t)}-1\right)\right)^{1 / 2}
$$

in which $\mathrm{T}$ is the desired temperature and $\mathrm{T}(\mathrm{t})$ is current temperature. $\tau_{\mathrm{T}}$ is a coupling parameter. Such a method can maintain the temperature, but cannot sample a canonical ensemble. Bussi et al. proposed a modified method ${ }^{21}$ by adding a stochastic term, thus remaining the canonical ensemble:

$$
\begin{gathered}
\mathrm{d} K(t)=(K-K(t)) \frac{\Delta t}{\tau_{T}}+2 \sqrt{\frac{K K(t)}{N_{f}}} \frac{d W(t)}{\sqrt{\tau_{T}}} \\
\alpha=\sqrt{\frac{K(t)+d K(t)}{K(t)}}
\end{gathered}
$$

Here the $\mathrm{K}$ is desired kinetic energy and $\mathrm{K}(\mathrm{t})$ is current kinetic energy, $\mathrm{W}(\mathrm{t})$ is a stochastic noise.

For pressure controlling the Berendsen barostat ${ }^{20}$ is usually used, the idea is to rescale the box-center-of-mass coordinates: 


$$
\begin{gathered}
\boldsymbol{r}_{i}^{\prime}=\chi^{1 / 3} \boldsymbol{r}_{i} \\
\chi=1-\kappa \frac{\Delta t}{\tau_{P}}(P-P(t))
\end{gathered}
$$

in which $\kappa$ is isothermal compressibility and $\tau_{P}$ is the coupling parameter. Like above, $\mathrm{P}$ is the desired pressure and $\mathrm{P}(\mathrm{t})$ the current pressure.

\subsection{Constraint algorithm}

To save the computational resource, we treat some bonds as frozen, that is, we fix their distance all along the simulation. In that way, the corresponding degree of freedom is ignored. Usually the high frequency hydrogen-containing bond is constrained. SHAKE ${ }^{22}$ is a widely used method based on Lagrange multiplier method in which the constraint for each bond is satisfied iteratively using Newton-Raphson method. After integration, the new distance between two atoms should satisfy:

$$
\left(\boldsymbol{r}_{i j}+\delta \boldsymbol{r}_{i j}\right)-d^{2}=0
$$

This equation can also be rewritten as:

$$
2 \mu_{i j} g_{i j}\left(\boldsymbol{r}_{i j}^{0} \boldsymbol{r}_{i j}\right)+\mu_{i j}^{2} g_{i j}^{2}\left(\boldsymbol{r}_{i j}^{0}\right)^{2}=d^{2}-\left(\boldsymbol{r}_{i j}\right)^{2}
$$

in which $\mathrm{d}$ is the constraint distance and $\mu_{12}$ the reduced mass. $\mathrm{g}_{12}$ can be treated as Lagrange multiplier, so that the modification to new position can be readily computed:

$$
\begin{gathered}
\delta^{k} \boldsymbol{r}_{i}=\frac{1}{m_{i}} g_{i j}\left(\boldsymbol{r}_{i}^{0}-\boldsymbol{r}_{j}^{0}\right) \\
\delta^{k} \boldsymbol{r}_{j}=-\frac{1}{m_{j}} g_{i j}\left(\boldsymbol{r}_{i}^{0}-\boldsymbol{r}_{j}^{0}\right) \\
\boldsymbol{r}_{i j}^{\prime}=\boldsymbol{r}_{i}+\sum_{k^{\prime}<k} \delta^{k^{\prime}} \boldsymbol{r}_{i}-\boldsymbol{r}_{j}-\sum_{k^{\prime}<k} \delta^{k^{\prime}} \boldsymbol{r}_{j}
\end{gathered}
$$


$\delta^{\mathrm{k}} \mathrm{r}_{\mathrm{i}}$ means the modification to ith atom with regard to kth constraint. The new $\mathrm{r}_{\mathrm{ij}}^{\prime}$ will replace $r_{i j}$ in Eq 1.17. This procedure is applied iteratively until the difference between two steps is under certain threshold.

Water is the most common molecules and appears almost in every simulation. So some constraint method specific for water, such as $\operatorname{SETTLE}^{23}$, is proposed. The idea is to move the integrated position of atoms in the plane parallel to the plane before integration.

\subsubsection{Coarse grained MD (CGMD)}

Atomic scale MD is straightforward and easy to apply, while for larger system using such refined scale MD is sometimes costly as well as unnecessary. CGMD was then proposed to treat several atoms as a collective part to facilitate the computational efficiency. For example, the DNA can be represented by 3 interaction sites: phosphate, sugar and base $\mathrm{e}^{24}$. In the meanwhile, the corresponding coarse grained force field was built up based on atomic force field. The success of CGMD depends much on the composition of collective parts and the validity of corresponding force field. The application of CGMD to DNA denaturation achieved good agreement to experimental results $^{24,25}$.

\subsubsection{Replica-exchange molecular dynamics (REMD)}

The time scale of molecular simulation is usually from nano second to sub micro second, which is too short for experimental to observe. Such shortcoming is especially obvious when dealing with conformational change. Hence how to extend the 
simulation time is a key point to achieve valid simulation result. Apart from using faster computer, some enhanced sampling methods can help us to search the phase space more efficiently within limited time, and REMD is one of them.

Replica exchange simulations seek to enhance the sampling in such scenarios by running numerous independent replicas in slightly different ensembles, and periodically exchanging the coordinates of replicas between the ensembles. Typically, the set of replicas is constructed so that one extreme of the set of replicas is the ensemble from which sampling is wanted, and the other extreme is one where barriers can be crossed more easily. The stochastic exchange, which is based on Metropolis criterion, occurs with a probability $\mathrm{P}$ which depends on the reference temperature of two replicas $\left(T_{1}, T_{2}\right)$ and their instantaneous potential energies $\left(U_{1}, U_{2}\right)^{26}$ :

$$
\begin{gathered}
P\left(T_{1} \leftrightarrow T_{2}\right)=\min \left(1, e^{\Delta}\right) \\
\Delta=\left(\beta_{1}-\beta_{2}\right)\left(U_{1}-U_{2}\right)
\end{gathered}
$$

where $\beta_{1}=1 /\left(k_{B} T_{1}\right)$ and $\beta_{2}=1 /\left(k_{B} T_{2}\right)$ in which $\mathrm{kB}$ is Boltzmann constant.

REMD is especially useful when the phase space is totally unknown, that is, we cannot predict which movement direction is more favorable for the system and which degree of freedom will suffer dramatic change. Many successful examples employing this method have been seen, such as the folding pathway of $\operatorname{protein}^{27}$ and G-quadruplex ${ }^{28}$. 


\subsubsection{Molecular docking simulation}

When dealing with ligand-receptor interaction, we are more interested in what orientation is preferred instead of how system achieves such orientation. In that way, the docking method is proposed to explore the most preferred binding mode ${ }^{29}$. The advantage of docking is that it allows ligand to move freely in binding pocket so that it's much faster to search the phase space than MD simulation, thus it has wide application for virtual screening and drug design. Currently docking simulation was always used as a tool to explore the stability, as well as the possible conformation, of DNA-complex system ${ }^{30}$. Here the algorithm and scoring function based on GOLD ${ }^{31}$ will be introduced.

\subsubsection{Search algorithm}

To search for the preferred binding mode (a pose in docking simulation), many algorithms were proposed. Here the commonly used genetic algorithm will be introduced. The idea is to generate bunch of initial poses of ligand according to the torsional distribution, among which certain number of most stable poses will be selected out. These selected ones will be further used to generate hybrid "offspring" with certain degree of mutation. Such process is repeated until the difference between two neighboring generations is under certain threshold.

\subsubsection{Scoring function}

The scoring function is used to predict the binding energy of certain pose. Different docking softwares adopt different function types, but the common point is to separate the total value into separate part, and the final result is the summation of all 
the possible terms. The default function in GOLD is CHEMPLP (piecewise linear potential) $)^{32}$ :

$$
\begin{array}{cc}
\text { fitness }_{P L P}=-\left(f_{P L P}+f_{\text {lig-clash }}+f_{\text {lig-tor }}+c_{\text {site }}\right) & \text { Eq } 1.23 \\
\text { fitness }_{\text {CHEMPLP }}=\text { fitness }_{P L P}-\left(f_{\text {hb }}+f_{\text {met }}\right) & \text { Eq } 1.24
\end{array}
$$

in which $f_{P L P}$ is a function equal to the pairwise summation of atoms between ligand and receptor, which is based on the atom type and distance of atom pair. $f_{\text {lig-clash }}$ is a function to avoid ligand clash. $\mathrm{f}_{\text {lig-tor }}$ gives the contribution from torsional angle. $c_{\text {site }}$ confines the search region inside the binding pocket. $f_{h b}$ is the contribution from hydrogen bonding and $\mathrm{f}_{\text {met }}$ from metal interaction.

\subsubsection{Quantum mechanics (QM) method}

Above mentioned MD, CGMD and docking simulation can be classified as molecular mechanics (MM), which is characterized by classical energy function. In MM the atom is treated as a classical particle with point charge and bond as harmonic spring, the total energy then can be calculated using classical energy formulas. However, to determine the force constant as well as equilibrium distance, angle et al., more microscopic QM method is required ${ }^{33}$. Besides, QM method was also used to explore the electronic stacking property of G-quadruplex ${ }^{34}$. Hybrid QM/MM method were used to investigate varieties of enzyme-catalyzed reactions and DNA charge-transport ${ }^{35}$. 


\subsubsection{Basic principle of Ab-initio method}

Nearly all the QM method mentioned here is based on (time-independent) Schrodinger equation:

$$
\mathrm{H} \Psi=\mathrm{E} \Psi
$$

Eq 1.25

in which $\mathrm{H}$ is the Hamiltonian operator and $\Psi$ is the wave function (ortbital) and $\mathrm{E}$ is the corresponding energy. When dealing with multi-electron system, however, we cannot solve Schrodinger equation directly. Instead, several approximations were made: first, the nucleus is supposed to be fixed (Born-Oppenheimer approximation); second, the effect from other electrons is treated as a mean field, that is, the electron correlation is ignored. Under such approximations the Hamiltonian can be written as a Fock operator $^{36}$.

$$
f_{i}=-\frac{1}{2} \nabla_{i}^{2}-\sum_{k}^{\text {nuclei }} \frac{Z_{k}}{r_{i k}}+V_{i}^{H F}
$$

here $\mathrm{Z}_{\mathrm{k}}$ is the charge of nuclei and $\mathrm{V}_{\mathrm{i}}^{\mathrm{HF}}$ the electron repulsion interaction. In practice, we choose a basis set to build the molecular orbital:

$$
\phi_{i}=\sum_{\mu} a_{\mu i} \varphi_{\mu i}
$$

in which Roman letter is the molecular orbital index and Greek letter the basis function index. With variational principle, we can get secular equation ${ }^{37}$ :

$$
\left|\begin{array}{cccc}
F_{11}-E S_{11} & F_{12}-E S_{12} & \cdots & F_{1 N}-E S_{1 N} \\
F_{21}-E S_{21} & F_{22}-E S_{22} & \cdots & F_{2 N}-E S_{2 N} \\
\vdots & \vdots & \ddots & \vdots \\
F_{N 1}-E S_{N 1} & F_{N 1}-E S_{N 1} & \cdots & F_{N N}-E S_{N N}
\end{array}\right|=0
$$

in which 


$$
\begin{array}{cc}
F_{\mu \nu}=\left\langle\mu\left|-\frac{1}{2} \nabla^{2}\right| v\right\rangle-\sum_{k}^{\text {nuclei }} Z_{k}\left\langle\mu\left|\frac{1}{r_{k}}\right| v\right\rangle+\sum_{\lambda \sigma} P_{\lambda \sigma}\left[(\mu \nu \mid \lambda \sigma)-\frac{1}{2}(\mu \lambda \mid v \sigma)\right] & \text { Eq } 1.29 \\
(\mu \nu \mid \lambda \sigma)=\iint \phi_{\mu}(1) \phi_{\nu}(1) \frac{1}{r_{12}} \phi_{\lambda}(2) \phi_{\sigma}(2) d \boldsymbol{r}(1) d \boldsymbol{r}(2) & \text { Eq } 1.30 \\
S_{\mu \nu}=\langle\mu \mid v\rangle & \text { Eq } 1.31
\end{array}
$$

in which $\mathrm{P}_{\lambda \sigma}$ is the density matrix element:

$$
P_{\lambda \sigma}=2 \sum_{i}^{\text {occupied }} a_{\lambda i} a_{\sigma i}
$$

We solve density matrix P iteratively until it's converged. Such method is called HF (Hatree-Fock) method.

\subsubsection{DFT (Density Functional Theory)}

In DFT we write the final energy as a functional of electron density ${ }^{38}$ :

$$
\begin{gathered}
E[\rho(\boldsymbol{r})]=\sum_{i}^{N}\left(\left\langle\chi_{i}\left|-\frac{1}{2} \nabla_{i}^{2}\right| \chi_{i}\right\rangle-\left\langle\chi_{i}\left|\sum_{k}^{\text {nuclei }} \frac{Z_{k}}{\left|\boldsymbol{r}_{i}-\boldsymbol{r}_{k}\right|}\right| \chi_{i}\right|\right) \\
+\sum_{i}^{N}\left\langle\chi_{i}\left|\frac{1}{2} \int \frac{\rho\left(\boldsymbol{r}^{\prime}\right)}{\left|\boldsymbol{r}_{i}-\boldsymbol{r}^{\prime}\right|} d \boldsymbol{r}\right| \chi_{i}\right\rangle+E_{x c}[\rho(\boldsymbol{r})] \\
\rho=\sum_{i=1}^{N}\left\langle\chi_{i} \mid \chi_{i}\right\rangle
\end{gathered}
$$

in which $\chi_{\mathrm{i}}$ is the Kohn-Sham orbital and $E_{x c}[\rho(r)]$ is called exchange-correlation energy which contains all the correction to kinetic energy and electronic repulsion. Usually it cannot be expressed explicitly; instead an empirical term is given. Similar with $\mathrm{HF}$, we can also solve the secular equation iteratively until density matrix becomes converged. But different from HF method, in DFT the functional is approximate while the theory is exact. In addition, DFT usually requires less 
computational time than HF method ${ }^{29}$, so it's also a widely used method.

\subsection{Recent progress in computational advances}

The past decades have seen dramatic progress of theoretical simulation of DNA, here some important work will be introduced.

\subsubsection{Refinement of force field}

The accuracy of force field is crucial to a valid simulation. Therefore the improvement of force field is always a hot topic for MD simulation. AMBER parm $99^{39}$ has been proved to be an excellent force field, but its defect is the instability when applied to multi-nanosecond scale. The corrected version to it, such as parmbsc $0^{33}$, allowed the simulation to reach longer time scale. Recently, the correction to $\chi$ and $\varepsilon / \zeta$ torsional angle $e^{40,41}$, as well as parmbsc $1^{42}$ can even stay valid for more than $100 \mu \mathrm{s}$. In addition, another family, CHARMM force field ${ }^{43}$, also shows wide application.

On the other hand, we should always keep in mind the shortcoming of classical force field: it's just an approximation to complex molecule system. Some research pointed out the overestimation of AMBER force field to base-pair stacking ${ }^{44}$. And neglect of polarization also leads to incorrectness ${ }^{45}$, such as inaccuracy of description of energy and dynamics of multiple ions inside the G-quadruplex ${ }^{46}$, underestimation of helical twist of duplex DNA ${ }^{47}$ and self-diffusion coefficient of ionic liquid ${ }^{48}$. The development of polarizable force field is undergoing ${ }^{49}$, which has been shown to give 
good representation in hundred nanosecond scale but requires much more computational resource at the same time.

\subsubsection{The external environmental impact on DNA structures}

DNA usually stays in aqueous solution, in which varieties of ions exist. To understand the interplay between DNA and ion atmosphere, lots of research has been conducted. Lavery et al. developed a mature method to analyze the ion population around $\mathrm{DNA}^{50}$; and Pan et al. reported the difference of ion distribution within different forms of DNA and RNA ${ }^{51}$. MacKerell et al. has shown the ion can lead to the changes in groove geometry ${ }^{52}$.

The related research has also been extended to some non-physiological environment. Scientists has shown DNA may suffer large conformational change when it goes from aqueous solution to organic solutions with lower or even no polarity ${ }^{14,53}$; at the same time, the interaction between organic salt, such as choline ${ }^{54}$ and imidazole ${ }^{55}$, also provoked much interests. What's more, the investigation of DNA with kinds of media, such as lipids ${ }^{56}$ and graphene ${ }^{57}$, broaden the perspective of DNA research significantly.

\subsubsection{The simulation of non-canonical DNA structures}

Along with dominating $\mathrm{B}$ form, several other forms of DNA also exist ${ }^{58}$. The transition between different forms is of great interests. Recently, the study of G-quadruplex has drawn lots of attention ${ }^{59}$ because of its crucial role for DNA replication. Series of research were conducted with regard to the conformational 
property $^{60}$, binding of ligand ${ }^{61}$ et al. Recently some artificial structures, such as DNA origami ${ }^{62}$, were also simulated using MD method.

\subsubsection{Modification to DNA bases}

Epigenetics studies the effect of base mutation to the expression of gene. Recently, the theoretical study of methylated cytosine has shown that methylation decreased the elastic property of DNA and further changed the binding mode of DNA around histone ${ }^{63,64}$. Besides, the oxidation and deamination of guanine is also investigated extensively ${ }^{65,66}$ because of its close relationship to gene repairing.

\subsubsection{DNA interacting with binding molecules}

The interactions between DNA and small drug or large protein are of high significance in biology, medicine and therapeutics. Theoretical study involves the investigation towards binding and dissociation pathway, as well as the recognition of protein towards specific or non-specific DNA sequence ${ }^{67,68}$. For larger system, MD simulation revealed that lysine acetylation may lead to the unfolding of chromatin ${ }^{69}$. Apart from the protein, the investigation of small metal molecules binding to DNA also attracted interests: besides varieties of docking simulations, the possible binding modes of copper complex towards DNA were also explored by MD method ${ }^{70}$.

In conclusion, the computational method has shown its impressive power for DNA study. Our group has been focusing on study of the interaction between DNA and small molecules, such as DNA charge transport (CT) in ionic liquid ${ }^{71}$, and the interaction between G-quadruplex and metal complex ${ }^{72}$. The experimental results 
provided much inspiration and perspective to tune the fine structure of DNA and thus adjust the properties of DNA upon appearance of small molecules. At the same time, it's also demanding to elucidate the mechanism behind the experimental findings. With such purpose in mind, series of theoretical study has been done and the results well illustrate the experimental findings, which will be discussed in detail in the coming chapters. 


\section{Reference}

(1) Hershey, A. D.; Chase, M. Independent Functions of Viral Protein and Nucleic Acid in Growth of Bacteriophage. J Gen Physiol 1952, 36 (1), 39.

(2) Watson, J. D.; Crick, F. H. C. Molecular Structure of Nucleic Acids - a Structure for Deoxyribose Nucleic Acid. Nature 1953, 171 (4356), 737.

(3) Campolongo, M. J.; Tan, S. J.; Xu, J. F.; Luo, D. DNA nanomedicine: Engineering DNA as a polymer for therapeutic and diagnostic applications. Adv Drug Deliver Rev 2010, 62 (6), 606.

(4) Luo, D. From biology to materials: engineering DNA and RNA for drug delivery and nanomedicine Preface. Adv Drug Deliver Rev 2010, 62 (6), 591.

(5) Modi, S.; Swetha, M. G.; Goswami, D.; Gupta, G. D.; Mayor, S.; Krishnan, Y. A DNA nanomachine that maps spatial and temporal $\mathrm{pH}$ changes inside living cells. Nat Nanotechnol 2009, 4 (5), 325.

(6) Zheng, H. N.; Xiao, M. Y.; Yan, Q.; Ma, Y. Z.; Xiao, S. J. Small Circular DNA Molecules Act as Rigid Motifs To Build DNA Nanotubes. J Am Chem Soc 2014, 136 (29), 10194.

(7) Hariri, A. A.; Hamblin, G. D.; Gidi, Y.; Sleiman, H. F.; Cosa, G. Stepwise growth of surface-grafted DNA nanotubes visualized at the single-molecule level. Nat Chem 2015, 7 (4), 295.

(8) Kuzuya, A.; Komiyama, M. DNA origami: Fold, stick, and beyond. Nanoscale 2010, 2 (3), 310.

(9) Shih, W. M.; Lin, C. X. Knitting complex weaves with DNA origami. Curr Opin Struc Biol 2010, 20 (3), 276.

(10) Han, D.; Kang, H. Z.; Zhang, T.; Wu, C. C.; Zhou, C. S.; You, M. X.; Chen, Z.; Zhang, X. B.; Tan, W. H. Nucleic Acid Based Logical Systems. Chem-Eur J 2014, 20 (20), 5866.

(11) Perez, A.; Luque, F. J.; Orozco, M. Frontiers in Molecular Dynamics Simulations of DNA. Accounts Chem Res 2012, 45 (2), 196.

(12) Wong, K. Y.; Pettitt, B. M. The Pathway of Oligomeric DNA Melting Investigated by Molecular Dynamics Simulations. Biophys J 2008, 95 (12), 5618.

(13) Gilad, Y.; Senderowitz, H. Docking Studies on DNA Intercalators. J Chem Inf Model 2014, 54 (1), 96.

(14) Noy, A.; Perez, A.; Laughton, C. A.; Orozco, M. Theoretical study of large conformational transitions in DNA: the B <-> A conformational change in water and ethanol/water. Nucleic Acids Res 2007, 35 (10), 3330.

(15) Parker, T. M.; Hohenstein, E. G.; Parrish, R. M.; Hud, N. V.; Sherrill, C. D. Quantum-Mechanical Analysis of the Energetic Contributions to pi Stacking in Nucleic Acids versus Rise, Twist, and Slide. J Am Chem Soc 2013, 135 (4), 1306.

(16) Dans, P. D.; Walther, J.; Gomez, H.; Orozco, M. Multiscale simulation of DNA. Curr Opin Struc Biol 2016, 37, 29.

(17) Frenkel, D.; Smit, B. Understanding molecular simulation : from algorithms to applications; Academic Press: San Diego, 1996.

(18) Pearlman, D. A.; Case, D. A.; Caldwell, J. W.; Ross, W. S.; Cheatham, T. E.; Debolt, S.; Ferguson, D.; Seibel, G.; Kollman, P. Amber, a Package of Computer-Programs for Applying Molecular Mechanics, Normal-Mode Analysis, Molecular-Dynamics and Free-Energy Calculations to Simulate the Structural and Energetic Properties of Molecules. Comput Phys 
Commun 1995, 91 (1-3), 1.

(19) Darden, T.; York, D.; Pedersen, L. Particle Mesh Ewald - an N.Log(N) Method for Ewald Sums in Large Systems. J Chem Phys 1993, 98 (12), 10089.

(20) Berendsen, H. J. C.; Postma, J. P. M.; Vangunsteren, W. F.; Dinola, A.; Haak, J. R. Molecular-Dynamics with Coupling to an External Bath. J Chem Phys 1984, 81 (8), 3684.

(21) Bussi, G.; Donadio, D.; Parrinello, M. Canonical sampling through velocity rescaling. J Chem Phys 2007, 126 (1).

(22) Ryckaert, J. P.; Ciccotti, G.; Berendsen, H. J. C. Numerical-Integration of Cartesian Equations of Motion of a System with Constraints - Molecular-Dynamics of N-Alkanes. J Comput Phys 1977, 23 (3), 327.

(23) Miyamoto, S.; Kollman, P. A. Settle - an Analytical Version of the Shake and Rattle Algorithm for Rigid Water Models. J Comput Chem 1992, 13 (8), 952.

(24) Prytkova, T. R.; Eryazici, I.; Stepp, B.; Nguyen, S. B.; Schatz, G. C. DNA Melting in Small-Molecule-DNA-Hybrid Dimer Structures: Experimental Characterization and Coarse-Grained Molecular Dynamics Simulations. J Phys Chem B 2010, 114 (8), 2627.

(25) Li, H. P.; Wang, Z. L.; Li, N. N.; He, X. H.; Liang, H. J. Denaturation and renaturation behaviors of short DNA in a confined space. J Chem Phys 2014, 141 (4).

(26) Okabe, T.; Kawata, M.; Okamoto, Y.; Mikami, M. Replica-exchange Monte Carlo method for the isobaric-isothermal ensemble. Chem Phys Lett 2001, 335 (5-6), 435.

(27) van der Spoel, D.; Seibert, M. M. Protein folding kinetics and thermodynamics from atomistic simulations. Phys Rev Lett 2006, 96 (23).

(28) Luo, D.; Mu, Y. G. Computational Insights into the Stability and Folding Pathways of Human Telomeric DNA G-Quadruplexes. J Phys Chem B 2016, 120 (22), 4912.

(29) Cramer, C. J. Essentials of computational chemistry : theories and models; 2nd ed.; Wiley: Chichester, West Sussex, England ; Hoboken, NJ, 2004.

(30) Ma, D. L.; Lai, T. S.; Chan, F. Y.; Chung, W. H.; Abagyan, R.; Leung, Y. C.; Wong, K. Y. Discovery of a drug-like G-quadruplex binding ligand by high-throughput docking. Chemmedchem 2008, 3 (6), 881.

(31) Jones, G.; Willett, P.; Glen, R. C.; Leach, A. R.; Taylor, R. Development and validation of a genetic algorithm for flexible docking. J Mol Biol 1997, 267 (3), 727.

(32) Korb, O.; Stutzle, T.; Exner, T. E. Empirical Scoring Functions for Advanced Protein-Ligand Docking with PLANTS. J Chem Inf Model 2009, 49 (1), 84.

(33) Perez, A.; Marchan, I.; Svozil, D.; Sponer, J.; Cheatham, T. E.; Laughton, C. A.; Orozco, M. Refinenement of the AMBER force field for nucleic acids: Improving the description of alpha/gamma conformers. Biophys J 2007, 92 (11), 3817.

(34) Lech, C. J.; Heddi, B.; Phan, A. T. Guanine base stacking in G-quadruplex nucleic acids. Nucleic Acids Res 2013, 41 (3), 2034.

(35) Kubar, T.; Elstner, M. A hybrid approach to simulation of electron transfer in complex molecular systems. J R Soc Interface 2013, 10 (87).

(36) Zerner, M. C. Perspective on "New developments in molecular orbital theory" - Roothaan CCJ (1951) Rev Mod Phys 23 : 69-89. Theor Chem Acc 2000, 103 (3-4), 217.

(37) Roothaan, C. C. J. New Developments in Molecular Orbital Theory. Rev Mod Phys 1951, 23 (2), 69.

(38) Kohn, W.; Sham, L. J. Self-Consistent Equations Including Exchange and Correlation Effects. 
Phys Rev 1965, 140 (4a), 1133.

(39) Cheatham, T. E.; Cieplak, P.; Kollman, P. A. A modified version of the Cornell et al. force field with improved sugar pucker phases and helical repeat. J Biomol Struct Dyn 1999, 16 (4), 845.

(40) Krepl, M.; Zgarbova, M.; Stadlbauer, P.; Otyepka, M.; Banas, P.; Koca, J.; Cheatham, T. E.; Jurecka, P.; Sponer, J. Reference Simulations of Noncanonical Nucleic Acids with Different chi Variants of the AMBER Force Field: Quadruplex DNA, Quadruplex RNA, and Z-DNA. $J$ Chem Theory Comput 2012, 8 (7), 2506.

(41) Zgarbova, M.; Luque, F. J.; Sponer, J.; Cheatham, T. E.; Otyepka, M.; Jurecka, P. Toward Improved Description of DNA Backbone: Revisiting Epsilon and Zeta Torsion Force Field Parameters. J Chem Theory Comput 2013, 9 (5), 2339.

(42) Ivani, I.; Dans, P. D.; Noy, A.; Perez, A.; Faustino, I.; Hospital, A.; Walther, J.; Andrio, P.; Goni, R.; Balaceanu, A.et al. Parmbsc1: a refined force field for DNA simulations. Nat Methods 2016, 13 (1), 55.

(43) Huang, J.; MacKerell, A. D. CHARMM36 all-atom additive protein force field: Validation based on comparison to NMR data. J Comput Chem 2013, 34 (25), 2135.

(44) Brown, R. F.; Andrews, C. T.; Elcock, A. H. Stacking Free Energies of All DNA and RNA Nucleoside Pairs and Dinucleoside-Monophosphates Computed Using Recently Revised AMBER Parameters and Compared with Experiment. J Chem Theory Comput 2015, 11 (5), 2315.

(45) Luque, F. J.; Dehez, F.; Chipot, C.; Orozco, M. Polarization effects in molecular interactions. Wires Comput Mol Sci 2011, 1 (5), 844.

(46) Gkionis, K.; Kruse, H.; Platts, J. A.; Mladek, A.; Koca, J.; Sponer, J. Ion Binding to Quadruplex DNA Stems. Comparison of MM and QM Descriptions Reveals Sizable Polarization Effects Not Included in Contemporary Simulations. J Chem Theory Comput 2014, $10(3), 1326$.

(47) Banas, P.; Mladek, A.; Otyepka, M.; Zgarbova, M.; Jurecka, P.; Svozil, D.; Lankas, F.; Sponer, J. Can We Accurately Describe the Structure of Adenine Tracts in B-DNA? Reference Quantum-Chemical Computations Reveal Overstabilization of Stacking by Molecular Mechanics. J Chem Theory Comput 2012, 8 (7), 2448.

(48) Tsuzuki, S.; Shinoda, W.; Saito, H.; Mikami, M.; Tokuda, H.; Watanabe, M. Molecular Dynamics Simulations of Ionic Liquids: Cation and Anion Dependence of Self-Diffusion Coefficients of Ions. J Phys Chem B 2009, 113 (31), 10641.

(49) Savelyev, A.; MacKerell, A. D. All- Atom Polarizable Force Field for DNA Based on the Classical Drude Oscillator Model. J Comput Chem 2014, 35 (16), 1219.

(50) Lavery, R.; Maddocks, J. H.; Pasi, M.; Zakrzewska, K. Analyzing ion distributions around DNA. Nucleic Acids Res 2014, 42 (12), 8138.

(51) Pan, F.; Roland, C.; Sagui, C. Ion distributions around left- and right-handed DNA and RNA duplexes: a comparative study. Nucleic Acids Res 2014, 42 (22), 13981.

(52) Savelyev, A.; MacKerell, A. D. Differential Impact of the Monovalent Ions Li+, Na+, K+, and Rb+ on DNA Conformational Properties. J Phys Chem Lett 2015, 6 (1), 212.

(53) Arcella, A.; Portella, G.; Collepardo-Guevara, R.; Chakraborty, D.; Wales, D. J.; Orozco, M. Structure and Properties of DNA in Apolar Solvents. J Phys Chem B 2014, 118 (29), 8540.

(54) Nakano, M.; Tateishi-Karimata, H.; Tanaka, S.; Sugimoto, N. Choline Ion Interactions with 
DNA Atoms Explain Unique Stabilization of A-T Base Pairs in DNA Duplexes: A Microscopic View. J Phys Chem B 2014, 118 (2), 379.

(55) Jumbri, K.; Rahman, M. B. A.; Abdulmalek, E.; Ahmad, H.; Micaelo, N. M. An insight into structure and stability of DNA in ionic liquids from molecular dynamics simulation and experimental studies. Phys Chem Chem Phys 2014, 16 (27), 14036.

(56) Bagai, S.; Sun, C. B.; Tang, T. Lipid-Modified Polyethylenimine-Mediated DNA Attraction Evaluated by Molecular Dynamics Simulations. J Phys Chem B 2014, 118 (25), 7070.

(57) Bobadilla, A. D.; Seminario, J. M. Assembly of a Noncovalent DNA Junction on Graphene Sheets and Electron Transport Characteristics. J Phys Chem C 2013, 117 (50), 26441.

(58) Ghosh, A.; Bansal, M. A glossary of DNA structures from A to Z. Acta Crystallogr D 2003, 59, 620 .

(59) Rebic, M.; Mocci, F.; Laaksonen, A.; Ulicny, J. Multiscale Simulations of Human Telomeric G-Quadruplex DNA. J Phys Chem B 2015, 119 (1), 105.

(60) Moradi, M.; Babin, V.; Roland, C.; Sagui, C. Reaction path ensemble of the B-Z-DNA transition: a comprehensive atomistic study. Nucleic Acids Res 2013, 41 (1), 33.

(61) Ricci, C. G.; Netz, P. A. Docking Studies on DNA-Ligand Interactions: Building and Application of a Protocol To Identify the Binding Mode. J Chem Inf Model 2009, 49 (8), 1925.

(62) Snodin, B. E. K.; Romano, F.; Rovigatti, L.; Ouldridge, T. E.; Louis, A. A.; Doye, J. P. K. Direct Simulation of the Self-Assembly of a Small DNA Origami. Acs Nano 2016, 10 (2), 1724.

(63) Carvalho, A. T. P.; Gouveia, L.; Kanna, C. R.; Warmlander, S. K. T. S.; Platts, J. A.; Kamerlin, S. C. L. Understanding the structural and dynamic consequences of DNA epigenetic modifications: Computational insights into cytosine methylation and hydroxymethylation. Epigenetics-Us 2014, 9 (12), 1604.

(64) Portella, G.; Battistini, F.; Orozco, M. Understanding the Connection between Epigenetic DNA Methylation and Nucleosome Positioning from Computer Simulations. Plos Comput Biol 2013, 9 (11).

(65) Kara, M.; Zacharias, M. Influence of 8-Oxoguanosine on the Fine Structure of DNA Studied with Biasing-Potential Replica Exchange Simulations. Biophys J 2013, 104 (5), 1089.

(66) Krepl, M.; Otyepka, M.; Banas, P.; Sponer, J. Effect of Guanine to Inosine Substitution on Stability of Canonical DNA and RNA Duplexes: Molecular Dynamics Thermodynamics Integration Study. J Phys Chem B 2013, 117 (6), 1872.

(67) Yonetani, Y.; Kono, H. Dissociation Free-Energy Profiles of Specific and Nonspecific DNA-Protein Complexes. J Phys Chem B 2013, 117 (25), 7535.

(68) Furini, S.; Barbini, P.; Domene, C. DNA-recognition process described by MD simulations of the lactose repressor protein on a specific and a non-specific DNA sequence. Nucleic Acids Res 2013, 41 (7), 3963.

(69) Collepardo-Guevara, R.; Portella, G.; Vendruscolo, M.; Frenkel, D.; Schlick, T.; Orozco, M. Chromatin Unfolding by Epigenetic Modifications Explained by Dramatic Impairment of Internucleosome Interactions: A Multiscale Computational Study. J Am Chem Soc 2015, 137 (32), 10205.

(70) Galindo-Murillo, R.; Garcia-Ramos, J. C.; Ruiz-Azuara, L.; Cheatham, T. E.; Cortes-Guzman, F. Intercalation processes of copper complexes in DNA. Nucleic Acids Res 2015, 43 (11), 
5364.

(71) Xuan Shuguang, Meng. Z. Y., Wu Xiangyang, Wong Jiun-Ru, Devi Gitali, Edwin Yeow Kok Lee, Shao Fangwei Efficient DNA-mediated Electron Transport in Ionic Liquids. ACS Sustainable Chemistry and Engineering 2016.

(72) He, L.; Chen, X.; Meng, Z. Y.; Wang, J. T.; Tian, K. Y.; Li, T. H.; Shao, F. W. Octahedral ruthenium complexes selectively stabilize G-quadruplexes. Chem Commun 2016, 52 (52), 8095. 


\section{Chapter 2}

The structural study of DNA in ionic liquid 


\subsection{Motivation: The study of DNA conformational change in IL}

The duplex structure of DNA possesses both stability and flexibility, for example, the $\mathrm{B} \leftrightarrow \mathrm{A}$ and $\mathrm{B} \leftrightarrow \mathrm{Z}$ second structure transition has been studied exhaustively ${ }^{1-5}$. Besides, upon binding of organic small molecules ${ }^{6}$ or metal complexes ${ }^{7}$, the subtle conformational change may also occur. However, due to the limitation of measurement accuracy, experimental study can only provide indirect indication for such change.

Apart from experimental study, in silico simulation provided a more microscopic perspective to study conformational change of DNA. Orozco et al. reported the B- to A-form transition in high concentration of ethanol/water solvent by MD simulation ${ }^{8}$. Sagui et al. explored the B $\leftrightarrow \mathrm{Z}$ transition energy curve and found different pathways for such transition ${ }^{9}$. The advantage of computational study of DNA conformation is that it can provide a quite precise description of the structural information, which may be unavailable by experimental approaches.

As above mentioned, the solvent plays a critical role to tune the conformation of DNA, which reminded scientists the possibility to explore some non-aqueous and non-organic solvents as a tool to fulfill this goal. With regard to this point, ionic liquid seems a promising candidate. Ionic liquid is an ideal solvent for bio-molecule storage because of its outstanding biocompatibility ${ }^{10}$ and electronic property ${ }^{11}$. It's further found that DNA can maintain duplex in choline salts under room temperature for nearly half a year ${ }^{12}$, which suggested IL can stabilize DNA conformation significantly. 
Furthermore, IL can also tune the conformation of duplex according to both experimental and simulation results ${ }^{13,14}$.

In their pioneering work, Senapati et al. explored the minor groove binding affinity of $[\mathrm{BMIM}]^{+}\left[\mathrm{BF}_{4}\right]^{-}$by both experimental and simulation method. It was found that $[\mathrm{BMIM}]^{+}$can intrude into the hydration spine and replace the water molecules of minor groove ${ }^{13}$. Later, Micealo et al. reported the effects from the length of side chain on imidazole cation ${ }^{15}$ and Sugimoto et al. found the same phenomenon for choline cation $^{16}$. Their work revealed the important interaction between cations and DNA minor groove. However, the influence of such binding on the DNA conformation lacks further investigation.

In our group's previous work, we found the DNA CT rate in $[\mathrm{BMIM}]^{+}\left[\mathrm{BF}_{4}\right]^{-}$was facilitated $^{17}$. The detail of experimental setup will be introduced in next chapter; here we will focus only on the structural change upon binding of ionic liquid. It's highly possible that the introduction of ionic liquid induced the subtle change of DNA conformation which can improve the $\mathrm{CT}$ rate, however by current experimental instruments such subtle conformational change is hard to detect. Up to this point, it's necessary to employ some theoretical method to explore the conformational change of base part to demonstrate the effect of ionic liquid.

\subsection{Methods}

\subsubsection{Simulation details}

In this study, two series of simulations, DNA in water and DNA in hydrated ionic 
liquid, were explored. For each series, 3 individual repeats with different initial velocity were conducted. The sequence used was $5^{\prime}-\mathrm{T}_{11}$ CCTTA-3'. Compared with the experimental sequence the tail part was cut to reduce the computational resource.

The B-form DNA duplex with the designed sequence was built up using $3_{\text {DNA }}{ }^{18,19}$. The force filed for DNA was AMBER99bsc0 ${ }^{20}$ with $\varepsilon / \zeta$ OL1 and $\chi$ OL4 modification $^{21,22}$. The prepared structure was then put into a cubic box with minimum distance between any DNA atom and box surface to be $1.2 \mathrm{~nm}$. For pure water environment, the box was filled up with TIP3P water molecules ${ }^{23}$. For hydrated ionic liquid environment, the box was first filled with 600 of $\mathrm{BMIM}^{+}$and $\mathrm{BF}^{4-}$ followed by TIP3P water molecules. The final concentration of IL was around $50 \%(\mathrm{w} / \mathrm{w})$ which was consistent with experimental settings. The force field of ionic liquid was based on the reference ${ }^{24}$ with atomic charge recalculated using RESP method at $\mathrm{HF} / 6-31 \mathrm{G}^{*}$ level.

The system then underwent minimization using steepest descent method until the maximum force is below $50 \mathrm{kJmol}^{-1} \mathrm{~nm}^{-1}$. The thermostat coupling to $298 \mathrm{~K}$ and barostat coupling to 1 atm was then introduced, with each equilibrium period to be 100 ps. The thermostat method was v-rescale ${ }^{25}$ and Berendsen barostat ${ }^{26}$ was used as pressure coupling method. Thereafter a $100 \mathrm{~ns}$ (in IL)/30 ns (in water) product MD with 2 fs time step was performed.

All the simulations were performed using GROMACS 4.6 package ${ }^{27-30}$. Periodic boundary condition (PBC) and particle meshed Ewald were employed for all the 
simulation, constraint for all H-containing bonds were achieved using LINCS method $^{31}$.

\subsubsection{Collective internal parameter vector}

To investigate the subtle conformational difference in different solvents, only comparing atom coordinates seemed tedious and unnecessary, since some intramolecular degrees of freedom, such as the vibration between atoms within one base, were hardly related to $\mathrm{CT}$; instead, the structural relationship between complementary bases, or neighboring bases, becomes quite critical. With such an idea in mind, we introduced internal parameter vector (IPV) as a collective parameter to analyze the conformation of base part. Under CEHS scheme ${ }^{32}, 6$ parameters (shear, stretch, stagger, buckle, propeller and opening) were used to describe each base pair and 6 parameters (shift, slide, rise, tilt, roll and twist) were used for each base step, as figure 2-1 shows. Such collective parameters reduce the complexity and thus are more straightforward. 

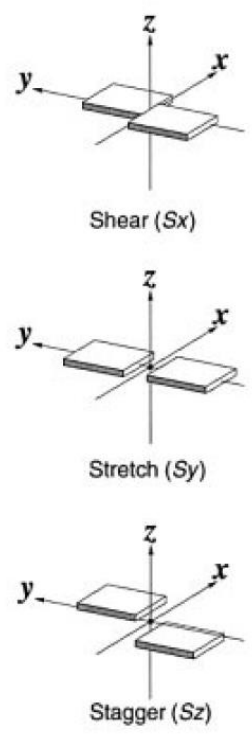
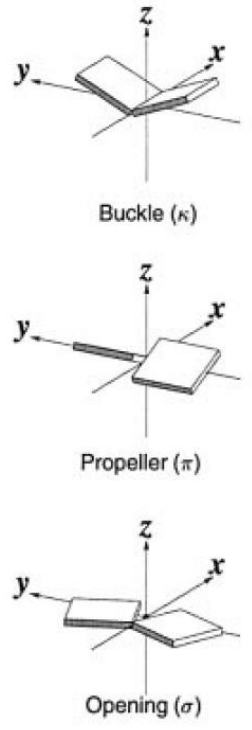
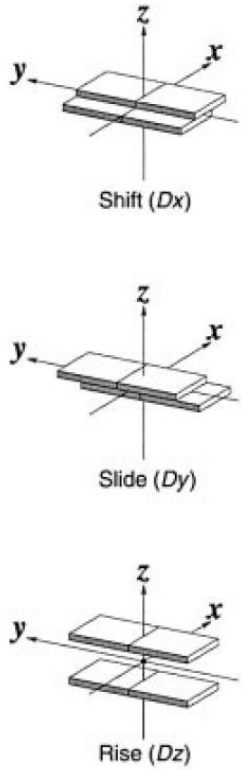
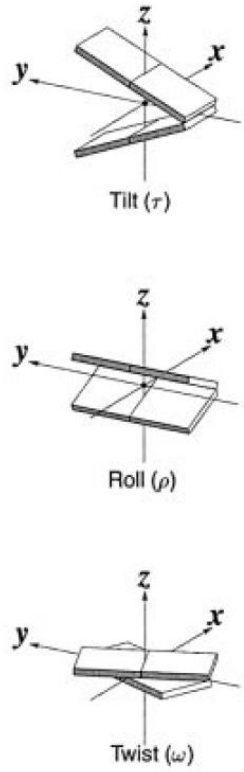

Figure 2-1 The definition of 6 base pair parameters (left 2 columns) and 6 base step parameters (right 2 columns). The graph was taken from ref 18

The last 10 ns trajectory of obtained product MD was extracted and each frame's base part coordinates were converted to internal parameter vector using 3DNA. The terminal base pair and base step were discarded because of their large fluctuation. Therefore each vector contained 162 elements (14 base pairs $\times 6$ base pair parameters and 13 base steps $\times 6$ base step parameters). In total we had 5000 IPVs for each simulation.

\subsubsection{Principal component analysis}

Principal component analysis is a mathematical method to simplify data complexity and illustrate data distribution pattern. For a set of $\mathrm{N}$-dimension vector $\mathrm{X}=\left[\mathrm{X}_{1}, \mathrm{X}_{2}, \cdots, \mathrm{X}_{\mathrm{N}}\right]$, its covariance matrix is defined as: 


$$
\begin{gathered}
\mathbb{C}=\left(\begin{array}{cccc}
c_{11} & c_{12} & \cdots & c_{1 N} \\
c_{21} & c_{22} & \cdots & c_{2 N} \\
\vdots & \vdots & \ddots & \vdots \\
c_{N 1} & c_{N 2} & \cdots & c_{N N}
\end{array}\right) \\
c_{i j}=E\left[\left(X_{i}-E\left(X_{i}\right)\right)\left(X_{j}-E\left(X_{j}\right)\right)\right]
\end{gathered}
$$

where $E\left(X_{i}\right)$ denotes the expected value of $X_{i}$. Since $c_{i j}=c_{j i}$, the matrix is symmetric and can thus be diagonalized. After diagonalization we will have eigenvalues and corresponding eigenvectors:

$$
\mathbb{C} \boldsymbol{E}_{i}=c_{i} \boldsymbol{E}_{i}
$$

The eigenvectors are perpendicular to each other, and the one with larger eigenvalue shows more obvious distribution direction. So usually first and second eigenvectors was chosen and all the vector $\mathrm{X}$ are projected to them:

$$
\begin{aligned}
& \boldsymbol{X} \boldsymbol{E}_{1}=P_{1} \\
& \boldsymbol{X} \boldsymbol{E}_{2}=P_{2}
\end{aligned}
$$

here $\mathrm{P}_{1}$ and $\mathrm{P}_{2}$ are the projected values of $\mathrm{X}$ to first and second eigenvectors, respectively. After projection, the dimensions of data are significantly reduced to 2 and can be visualized using a plane map.

The IPVs were subject to principal component analysis (PCA). The eigenvectors were determined from all the IPVs involved (5000 IPVs for each repeat, 3 repeats in water, 3 repeats in hydrated ionic liquid). The IPVs of either single repeat or all 3 repeats were projected to the plane formed by first and second eigenvectors. The plane was divided into $40 \times 40$ grids and the projected points in each grid were counted. The population was then converted to corresponding free energy according to 
Boltzmann formula. The distribution patterns were drawn using contour method.

The projected points from central region of distribution map were filtered out and the averages of the corresponding IPVs, $\overline{\mathrm{IPV}} \mathrm{s}$, were calculated. The difference between central IPVs were computed and compared.

\subsubsection{Clustering and visualization}

The frames corresponding to filtered IPVs were clustered using single-linkage method and the central structure of the cluster with largest population was visualized using VMD (http://www.ks.uiuc.edu/Research/vmd/) $)^{33}$.

\subsubsection{Contact number of cations towards minor groove base}

The stable residence of cation in DNA minor groove has been reported by previous studies ${ }^{13,15}$. The binding mode of cations can be illustrated using contact number. For each frame, the cation atoms which stand within $0.25 \mathrm{~nm}$ from minor groove atoms of each base pair ( $\mathrm{N} 3$ of adenine, $\mathrm{O} 2$ of thymine, $\mathrm{O} 2$ of cytosine and $\mathrm{N} 3$, H22 of guanine) were count and the average value (contact number) over certain period were computed.

\subsubsection{Overlap area of base step}

The overlap areas between base pairs were calculated using 3DNA under CEHS scheme $^{32}$. The basic idea is to find a mid-step triad (MST) between two neighboring base pairs and the atom coordinates of them are then projected to the $\mathrm{x}-\mathrm{y}$ plane of MST and the overlap area are calculated. 


\subsection{Results and discussion}

\subsubsection{The subtle conformational change in IL}

First of all, the RMSD for all the simulations were calculated and the results were shown in figure 2-2. All the simulations gave the RMSD value below $0.4 \mathrm{~nm}$, which suggested the stability of the system. The different averaged RMSD value of DNA in IL and water suggested the subtle conformational change of DNA, and the less standard deviation implied DNA in water suffered less fluctuation, as shown in table $2-1$.
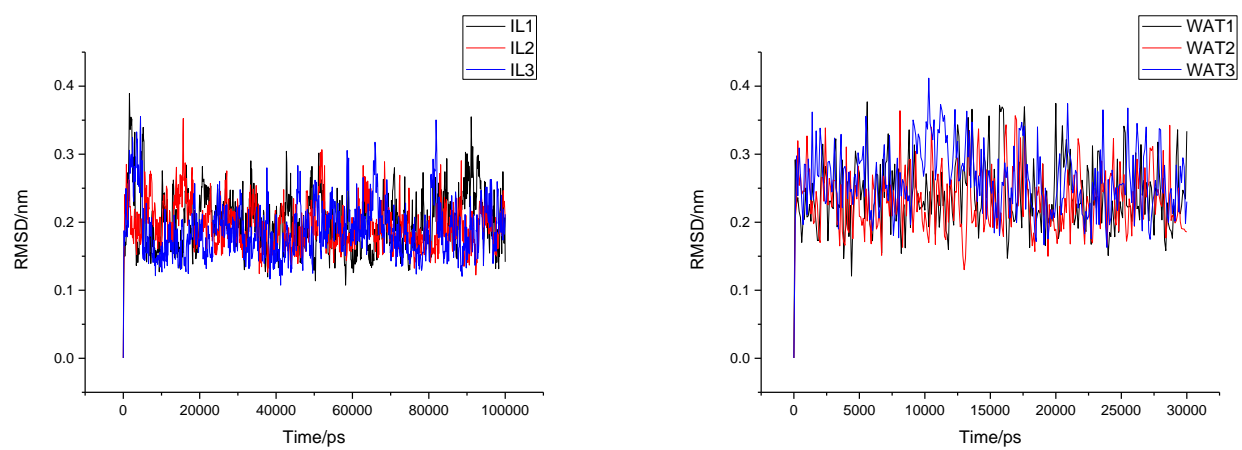

Figure 2-2 The RMSD of DNA in IL and in water, with the initial structure as reference.

Table 2-1 The average and standard deviation of RMSD value in different environment.

\begin{tabular}{ccc}
\hline & IL/nm & Water/nm \\
\hline $\mathbf{1}$ & $0.2009 \pm 0.0427$ & $0.2394 \pm 0.0506$ \\
& & \\
$\mathbf{2}$ & $0.1956 \pm 0.0337$ & $0.2314 \pm 0.0461$ \\
\hline
\end{tabular}


Then PCA was conducted using the last 10 ns trajectory of each simulation. The IPVs from all 3 simulation repeats in water and in IL were projected onto the plane formed by the first and second eigenvectors of PCA. The population in each grid was counted and the projection map was drawn using contour method (Figure 2-3). The conformation distribution of DNA showed distinct images between water and hydrate IL. In water the conformation of duplex DNA showed an extended circle region with the center located at $(0.54,0.04)$. While in IL, two local minimum appeared. The shallower minimum located at $(-0.09,-0.41)$ and the deeper one focused at $(0.62$, 1.01). Such a difference in conformation contour indicated that upon binding of IL molecules the dynamic conformation of DNA helixes became more confined.

To further analyze such discrepancy, the IPVs of each individual repeat were projected to the same plane separately. For water case, 3 repeats showed rather similar distribution with $x$ value went from -0.75 to 1.50 and $y$ value from -1.0 to 1.2 . Such a large distribution indicated that the DNA in water was quite flexible, and the consistent pattern of different repeats showed the phase space surface was quite smooth. With different starting points, the conformation of duplex DNA can always achieve the same energy minimum.

On the contrary, the DNA in IL showed quite different picture. Apart from the different local energy minimum, the area of distribution was smaller compared with 
that in water. We further noticed the upper minimum was from the first repeat, whereas the lower minimum was obtained from the other two repeats. For the second and third repeats, the distribution showed a narrow and oblique distribution pattern with $\mathrm{x}$ value from -1.0 to 0.75 and $\mathrm{y}$ value from -1.25 to 0.5 . The smaller distribution area indicated the fluctuation of DNA conformation in IL was confined. To further illustrate such phenomenon, we computed the entropy of nucleobases using quasi-harmonic method ${ }^{34}$. As shown in table 2-2, the entropy in IL gave the value around $600 \mathrm{Jmol}^{-1} \mathrm{~K}^{-1}$ less than that in water, which confirmed the stability of DNA in IL.
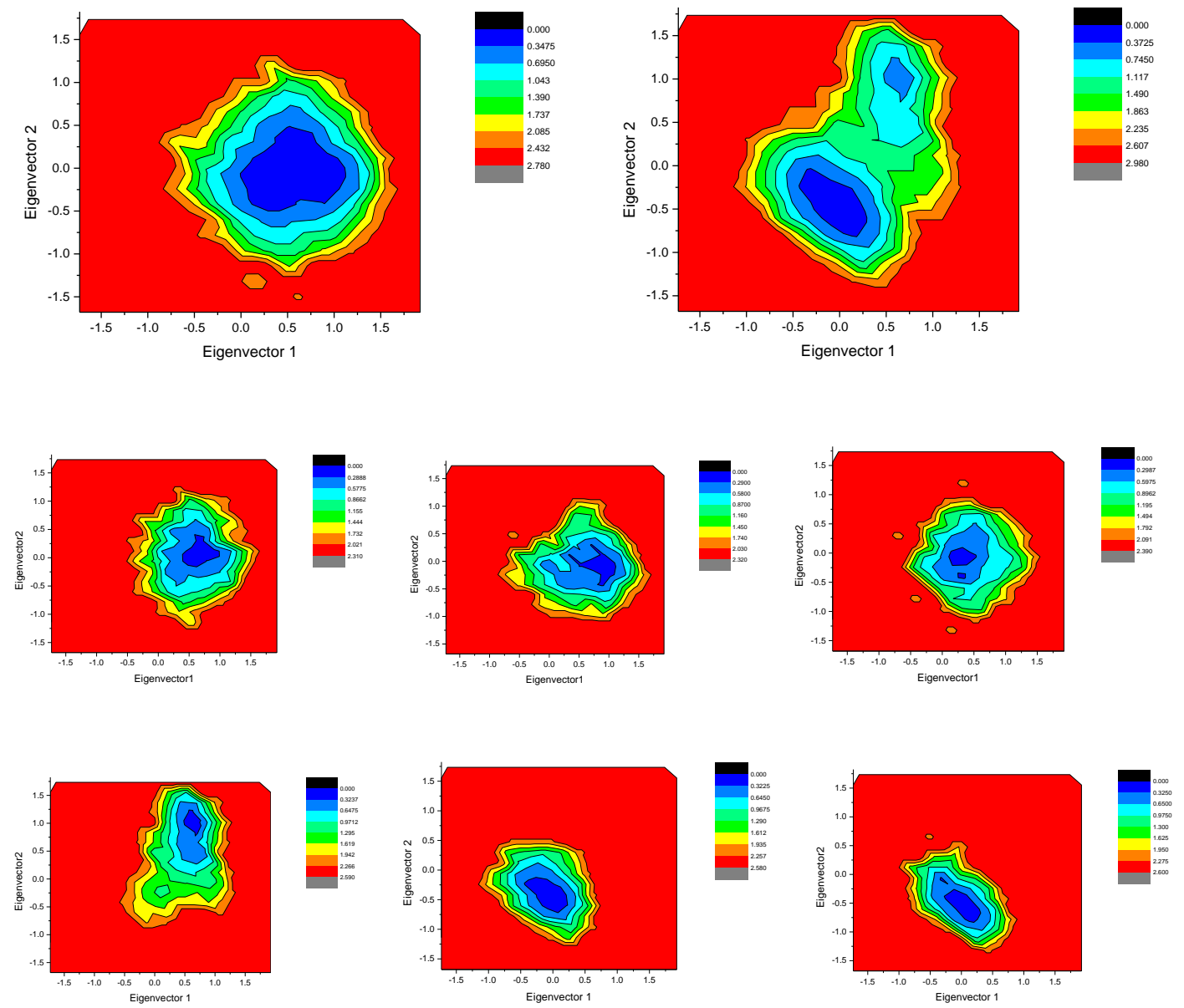

Figure 2-3 The PCA projection maps. Top left: overlap of 3 water repeats; top right: 
overlap of $3 \mathrm{IL}$ repeats; central: 3 individual water repeats; bottom: 3 individual IL repeats.

Table 2-2 The computed entropy in different environments

\begin{tabular}{cc}
\hline & Quasi-harmonic entropy $(\mathbf{J} / \mathbf{m o l K})$ \\
\hline IL1 & 3124.33 \\
IL2 & 3052.13 \\
IL3 & 3245.38 \\
Average & 3140.61 \\
WAT1 & 3810.42 \\
WAT2 & 3706.06 \\
WAT3 & 3682.41 \\
\hline Average & 3732.96 \\
\hline
\end{tabular}

\subsubsection{C-G and its proximal region showed more conformational} change

The difference in projection map was then further analyzed to identify the part of duplex DNA which suffered the most conformational change. The points located within the central region (center \pm 0.1 ) of conformational map for each repeat was filtered out and the average of corresponding IPVs, $\overline{\mathrm{IPV}_{\mathrm{x}, \mathrm{filt}} \text {, }}$, was determined. These averaged IPVs were representative because they were most popular in projection map statistically. Subsequently the absolute value of the difference between two environments, $\left|\overline{\mathrm{IPV}_{\text {wat,filtered }}}-\overline{\mathrm{IPV}_{\mathrm{IL}, \mathrm{filtered}}}\right|$, was computed, as shown in figure 2-4. Because the conformation distribution map of water showed only one minimum, the 
IPVs were selected directly from the superimposed map. Comparing the figures for base pair and base step, it's clear that the stacked values of base pair parameters were much smaller than those of base step parameters. Besides, the differences between base pairs were moderate, with the most value of $\sim 0.4$. On the other hand, for base step parameters, the biggest difference can raise up to $\sim 1.0$. These discrepancies indicated the IL may mainly influence the interaction between base steps instead of complementary base pairs. With regard to base step parameters, we noticed some values were significantly affected by binding of IL, among them the largest values located in G-C and their neighboring T-A pairs with largest value going up to $~ 1.5$ and the most affected parameters were shift, tilt and twist. Interestingly, consistent with the different pattern of conformational map between the first repeat and the other two repeats in IL, the filtered IPVs also showed obvious different pattern: for IL repeat 1, the largest stacked value appears at CC/GG step; while for the other two it goes one step right to CT/AG step.

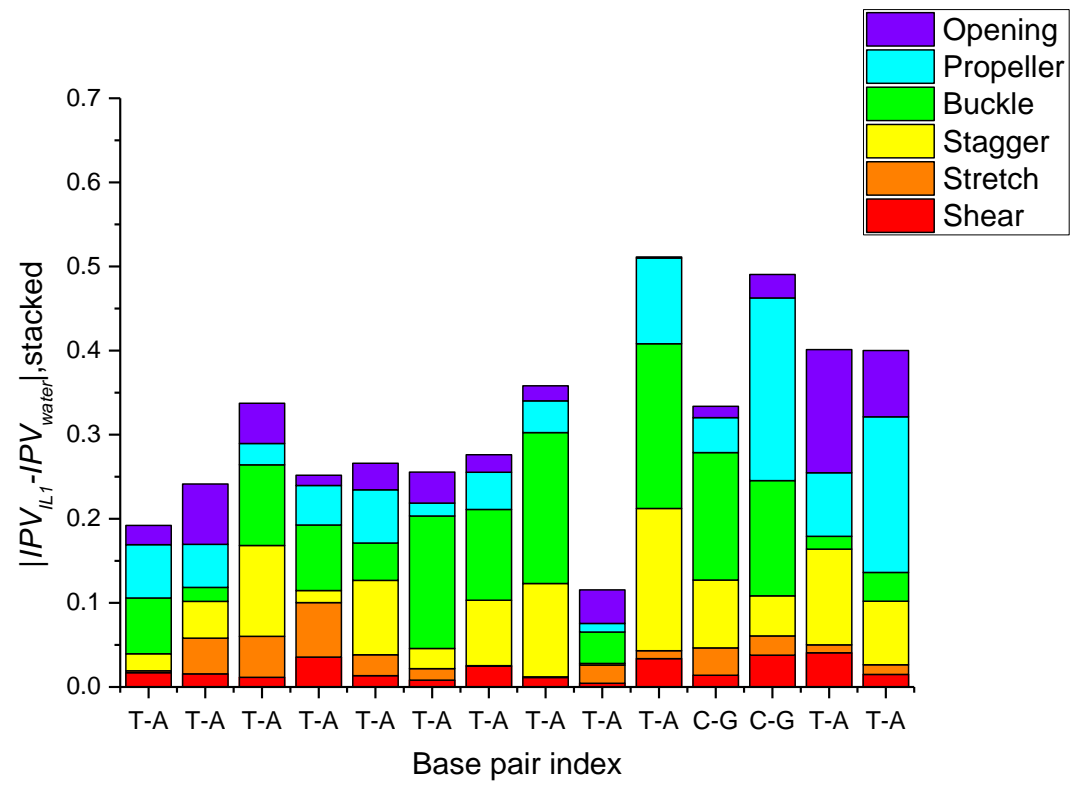



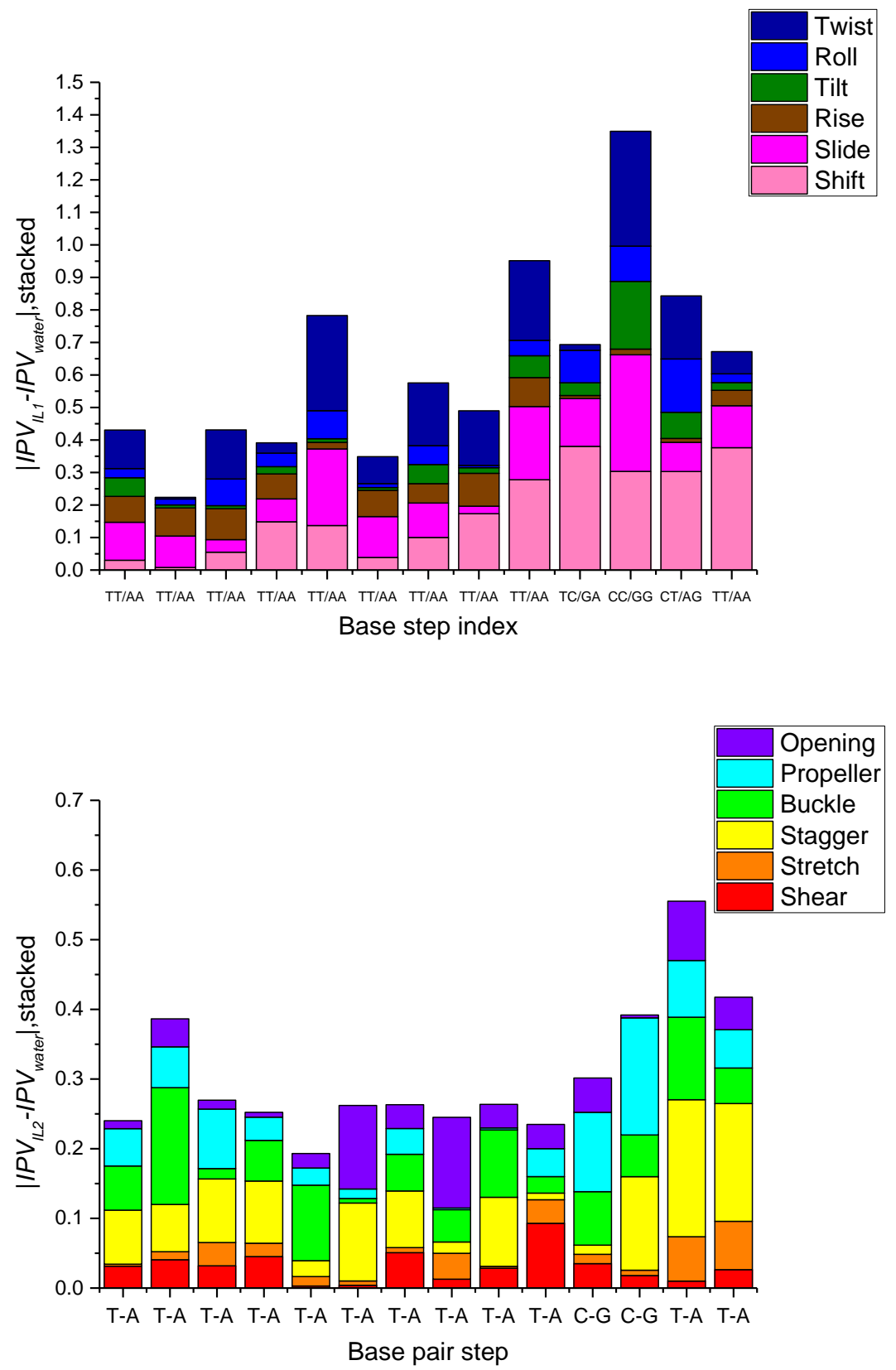

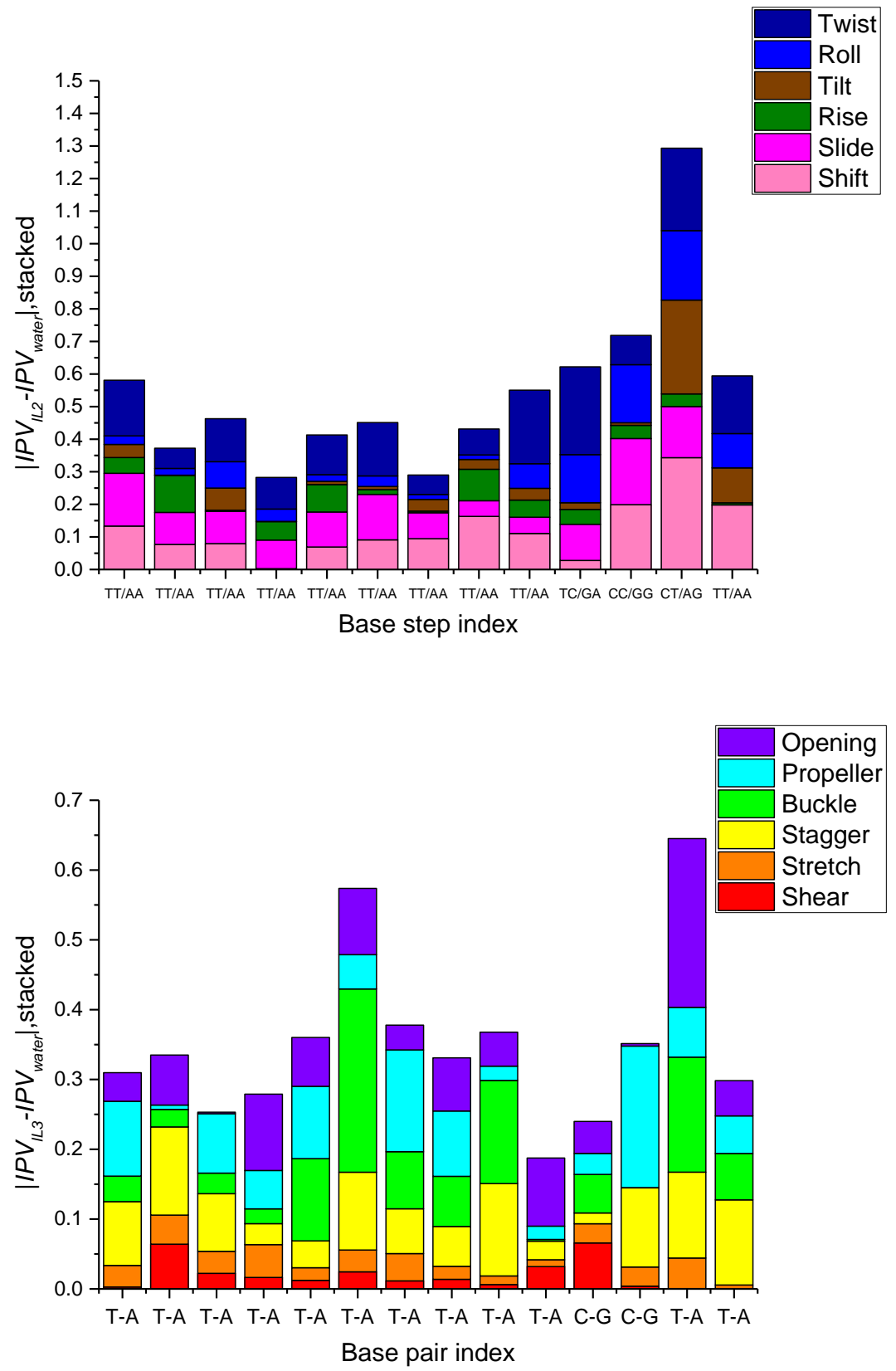


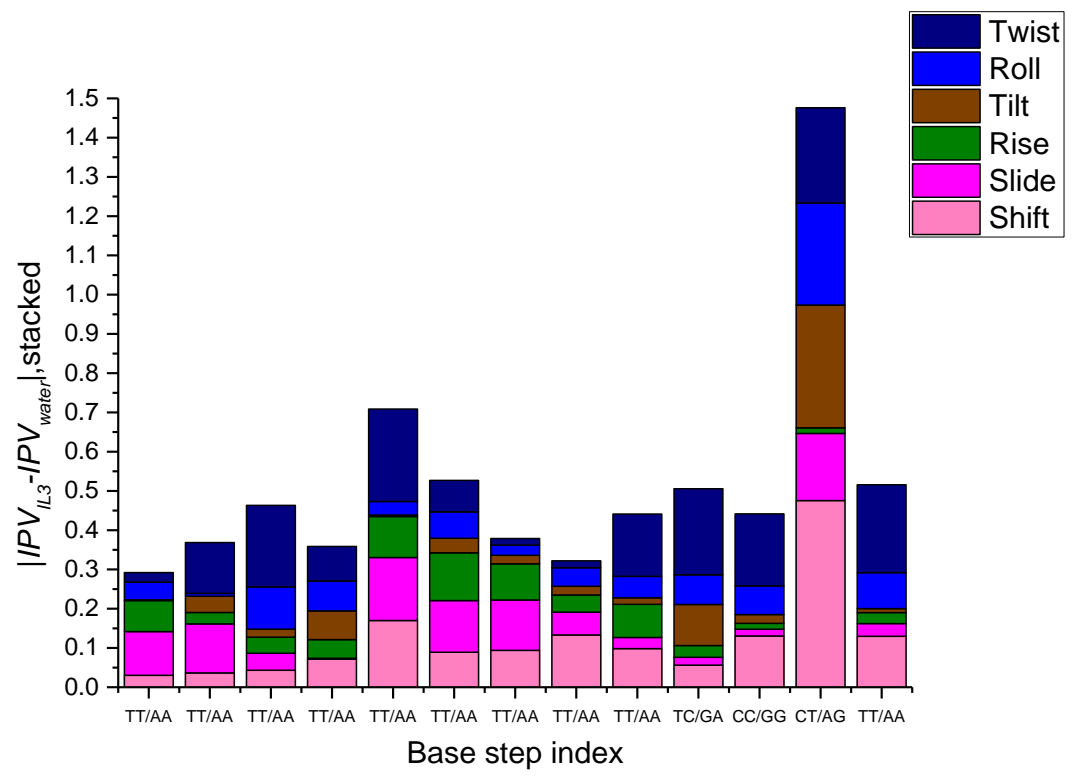

Figure 2-4 The absolute value of difference between filtered IPVs of water and IL.

\subsubsection{Binding of cations to minor groove induced conformational}

\section{change}

The difference between parameters came from the binding of IL molecules. To confirm this, the average contact number over last $10 \mathrm{~ns}$ was plotted for all 3 repeats of duplex DNA in IL (figure 2-5). Both the contact number of G-C pairs in repeat 2 and first G-C pair in repeat 3 went up to 1.2, while the rest of base pairs showed the contact number below 1.0. Such pattern showed the cations have higher affinity towards G-C pairs. However, repeat 1 showed no peak at G-C region, with contact numbers along the whole duplex between $0.4 \sim 0.8$. The binding of IL cations also influence the hydrogen bonding between water and DNA. As shown in table 2-3, in IL the number of hydrogen bonds is less than that in water, indicating some water nearby DNA is replaced by IL.

Table 2-3. The average number of hydrogen bonds in different solvents. The cut-off 
distance between $\mathrm{H}$ and acceptor was set to be $0.3 \mathrm{~nm}$ and the cut-off angle to be 30 degree.

\begin{tabular}{ccc}
\hline & IL & Water \\
\hline $\mathbf{1}$ & $231 \pm 9$ & $309 \pm 8$ \\
$\mathbf{2}$ & $229 \pm 8$ & $309 \pm 8$ \\
$\mathbf{3}$ & $230 \pm 8$ & $312 \pm 8$ \\
\hline
\end{tabular}

The influence of binding of cations also reflected on the change of overlap area between base pairs, as shown in figure 2-6. Different from smooth and consistent curves in water, the overlap area in IL showed rather significant fluctuation. Repeat 1 of hydrated IL showed a sharp peak $\left(>4 \AA^{2}\right)$ at TC/GA step. However this sharp peak appeared at neighboring CC/GG step in repeat 2 and 3. Again, such difference was also related to the binding pattern of cations.

With visualization, the binding pattern of IL cations can be illustrated more clearly. The frames in IL repeats corresponding to filtered IPVs were extracted in which the DNA and minor groove cations were clustered using single-linkage method followed by the visualization of the center structures of the main cluster, as shown in figure 2-7. The cations all resided in the minor groove which may be due to the electrostatic interaction between the cations and minor groove atoms (figure 2-8). In repeat 1, $[\mathrm{BMIM}]^{+}$took a head (imidazole ring) down/tail (alkyl chain) up pose and in repeat 2 
and 3 the direction became opposite. In Figure 2-6, all 3 repeats have a cation always appearing above G-C pair. For repeat 2 and 3, a cation was also observed at CT/AG step, while for IL repeat 1 , this cation went down to bottom TT/AA step. We attributed such different binding modes of cations to the cause of conformational change of DNA. It should be noted that 3 repeats may not exhaust all the possibilities of the conformational distribution as well as of the binding mode, the matches between IL repeat 2 and 3, however, indicated to some extent the consistency of the response of DNA upon binding of IL cations.

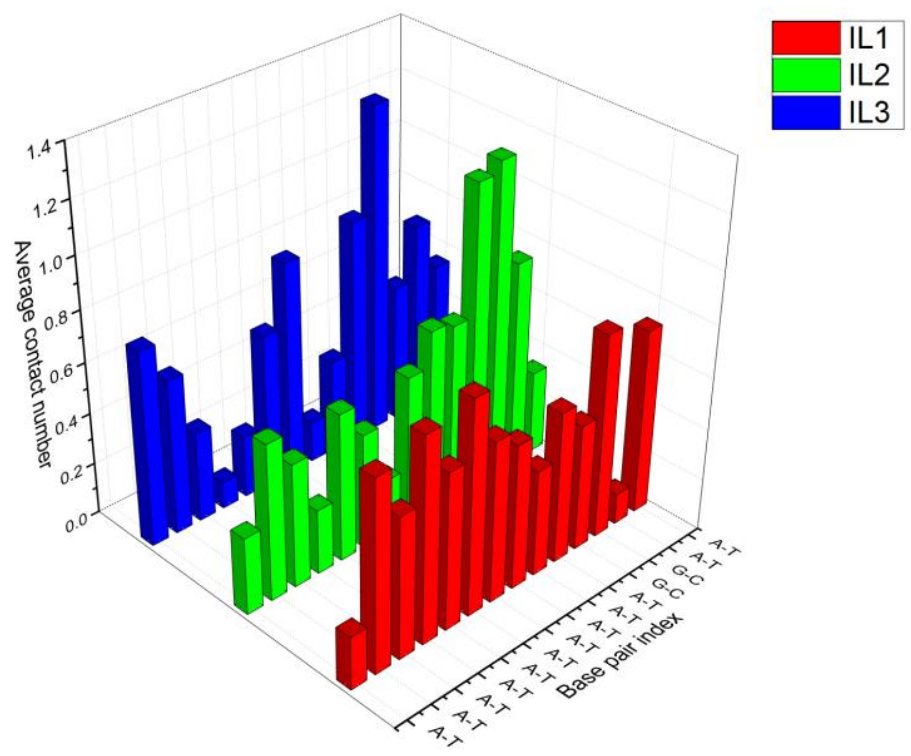

Figure 2-5 Average contact number of BMIM towards each site of minor groove, in last $10 \mathrm{~ns}$. 

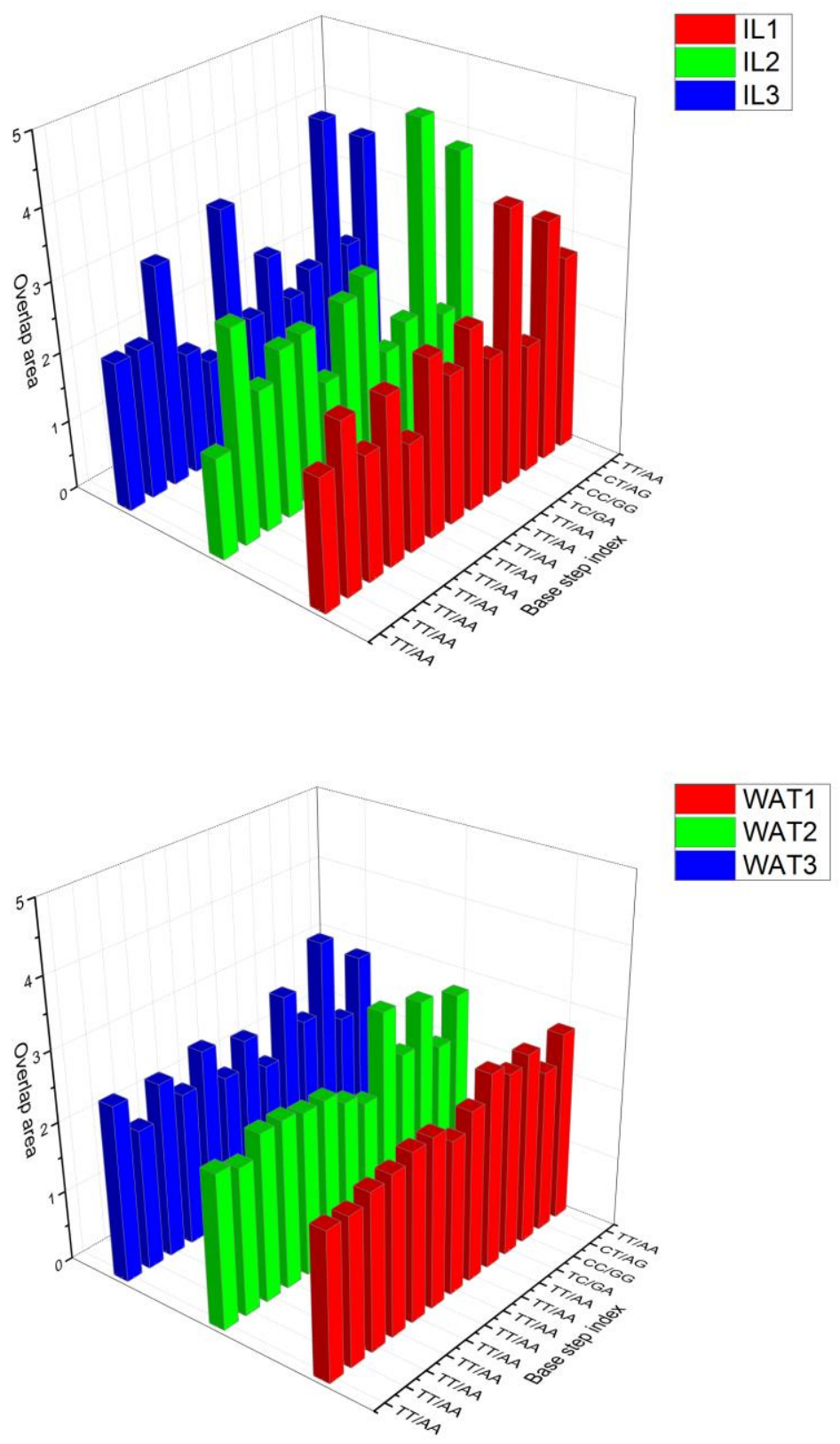

Figure 2-6 Overlap area (in $\AA^{2}$ ) of base steps in different solvents 

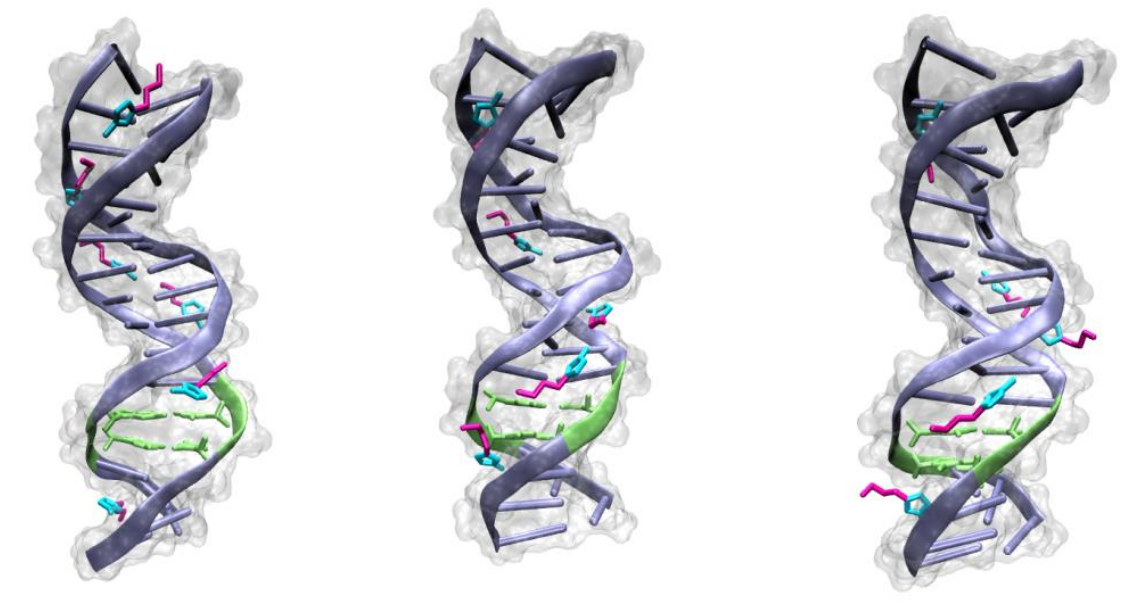

Figure 2-7 Representative structure of DNA and binding cations. Green: G-C pair; purple: A-T pair; cyan: imidazole ring (head); magenta: alkyl chain (tail).

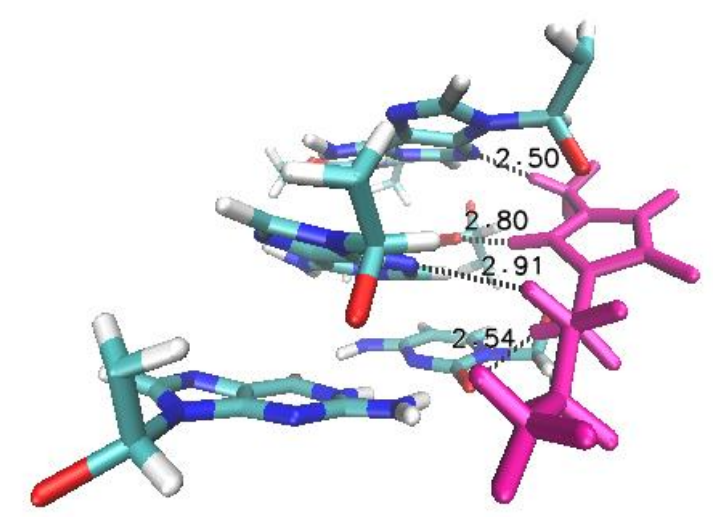

Figure 2-8 A zoom-in of cation-base electrostatic interaction. The graph was taken from representative structure of IL repeat 3. Blue: nitrogen; cyan: carbon; red: oxygen; white: hydrogen. The distance was measured in $\AA$.

\subsubsection{Dynamic motion of DNA induced by the change of binding mode}

Though IL can confine DNA conformation efficiently, the large conformational transition still occurred occasionally. Interestingly, such transition was also highly related to binding mode. From the distribution map of IL repeat 1, one can find a main 
local minimum located in the region with the second projection value of 1.0; apart from this main minimum, there's also a second minimum located in the middle of the map which is close to the position of main local minimum of repeat simulation $2 / 3$. Such a distribution suggested the conformation of DNA bases may undergo a dynamic change during the trajectory. To trace such process, we divided the last $10 \mathrm{~ns}$ trajectory into 10 windows, each window spans a $1 \mathrm{~ns}$ trajectory. The projections of each window onto the plane constructed by the first two eigenvectors are shown in Figure 2-9.

From the projection map of each window, it was easy to find out that during the $10 \mathrm{~ns}$ trajectory, the conformation traveled through at least 4 regions, region $1(0.5$, $0.5)$, region $2(0.75,0.0)$, region $3(0.0,-0.5)$ and region $4(0.75,1.0)$, respectively. At first the conformation stayed at region $1(90-91 \mathrm{~ns})$ and fluctuated to region 2 for a while (92-93ns), then soon come back to region 1 (93-94ns), after which an obvious transition happened and conformation went to region 3 (94-95ns). Please note the location of region 3 was quite close to where the minimum of repeat 2 and repeat 3 located. Finally the distribution went to region 4 and stayed there steadily (96-100ns). If the DNA conformation is represented by the 4 regions on the projection plane, the conformational change of DNA in the IL repeat 1 simulation can be summarized as: Region $1 \rightarrow 2 \rightarrow 1 \rightarrow 3 \rightarrow 1 \rightarrow 4$.

As mentioned above, region 3 located in the same region as the local minima of IL repeat 2 and 3 . The detailed structural patterns of region 3 were checked. The trajectories of 94.6-95.6 ns and 99-100 ns of IL repeat 1 is extracted, which 
corresponds to the conformation in region 3 and 4 respectively. The contact number as well as overlap area of both windows was shown in figure 2-10. We noticed that both the contact number and overlap area during 94.6-95.6 ns were quite similar to those of IL repeat 2 and 3: for contact number, G-C region showed a larger value (>0.9); for overlap area, the peak move to CC/GG step. While during the 99-100 ns, the pattern were more close to the $10 \mathrm{~ns}$ averaged ones in section 2.4.3. Such difference in contact number and overlap area during different periods actually matched the change in the projection map.

The relationship between DNA base conformation and cation binding was further confirmed by visualization at different time. In Figure 2-11 at t=90.0 ns, two BMIMs (yellow and green) resided around $\mathrm{C}-\mathrm{G}$ region with both head groups pointing to the GC base pairs. At $\mathrm{t}=92.5 \mathrm{~ns}$ both cations left minor groove region, such region is replaced by water again and such binding mode corresponded to the region 2 in the projection map. At $t=94.9 \mathrm{~ns}$ a new cation (pink) intrudes in the minor groove with its tail interacting with C-G pair and the green cation also came back and shifted down a little to position its head between T-A and G-C, as in repeat 2 and 3, thus increasing the contact number in such region. At $\mathrm{t}=99.1 \mathrm{~ns}$ the green cation shifted up again. The cations binding to upper T-A region (magenta ones) remained stable all along $10 \mathrm{~ns}$ trajectory. For repeat 2 and 3, we didn't observe the change of binding mode in minor groove cations. 

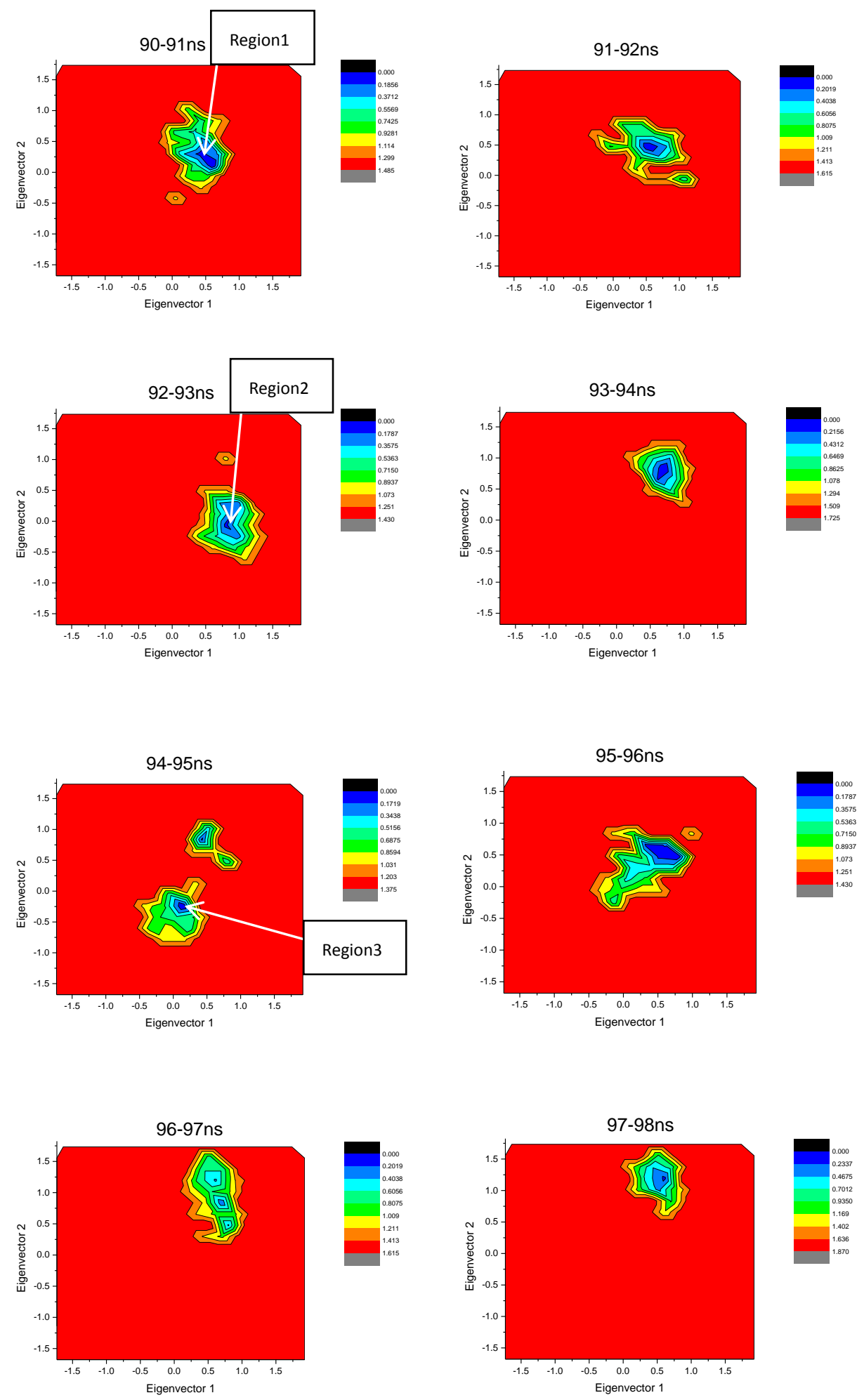

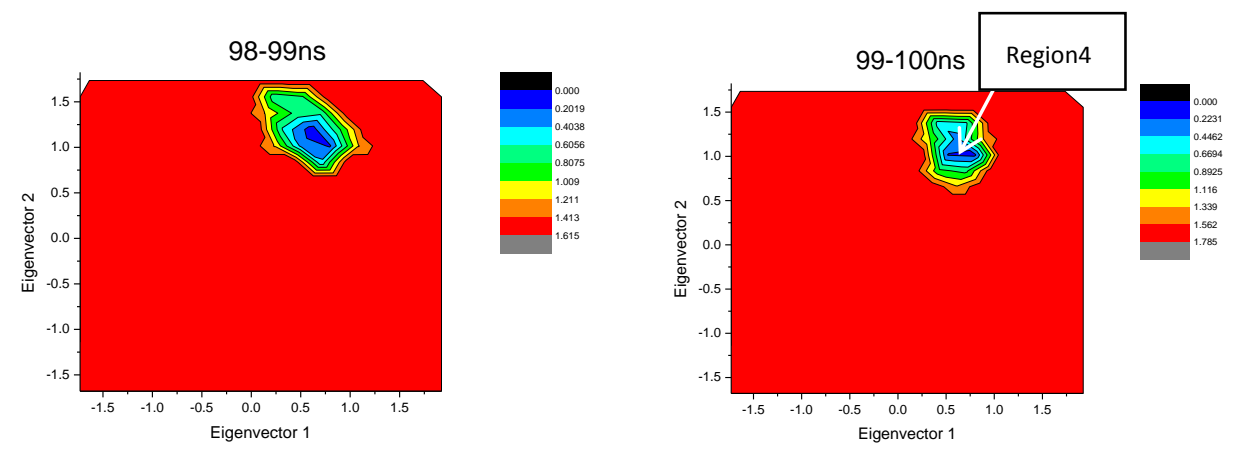

Figure 2-9 Dynamic process of IL repeat 1.4 regions in the map were marked.
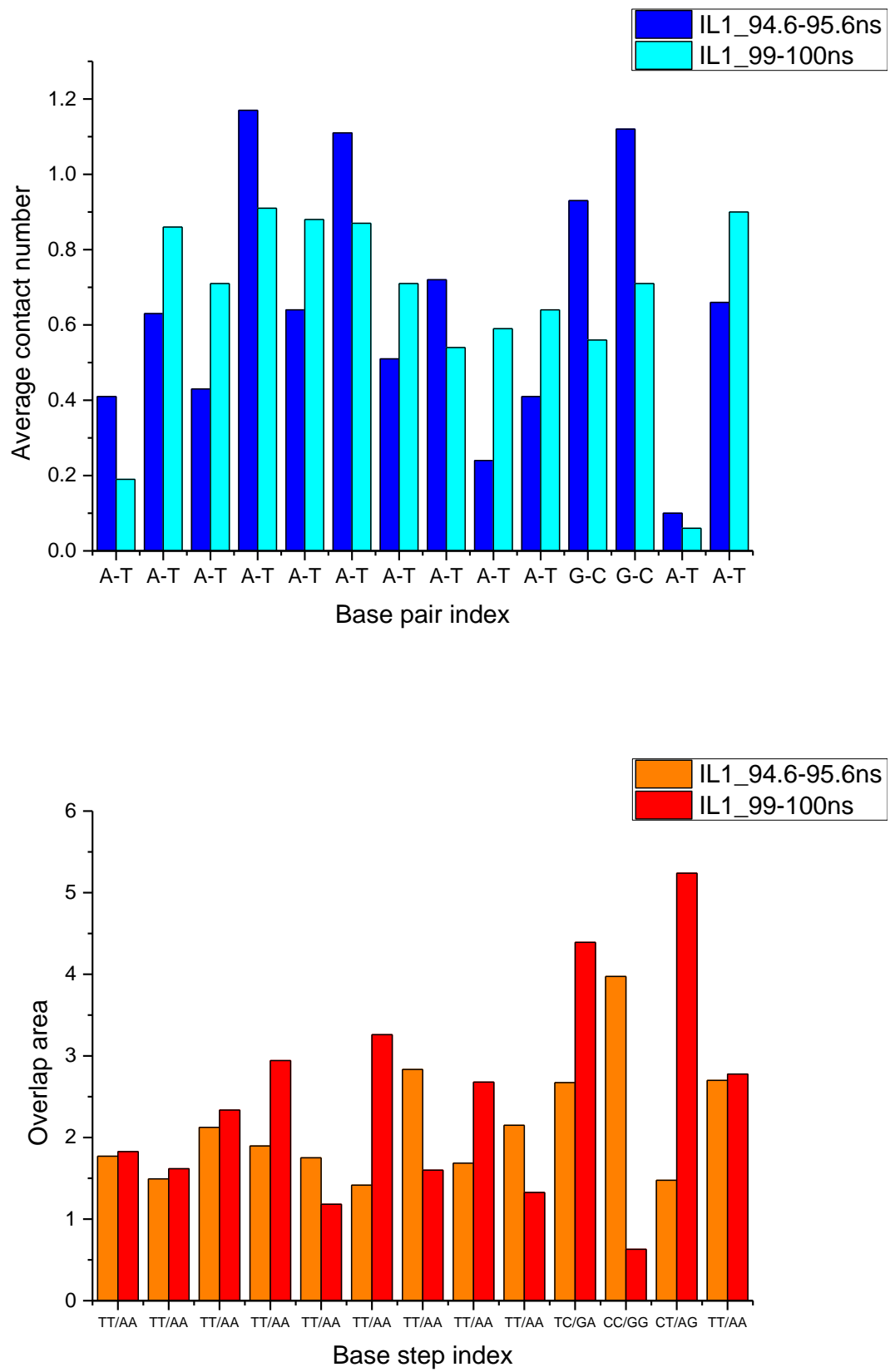
Figure 2-10 Contact number pattern and overlap area of IL repeat 1 in different time periods.
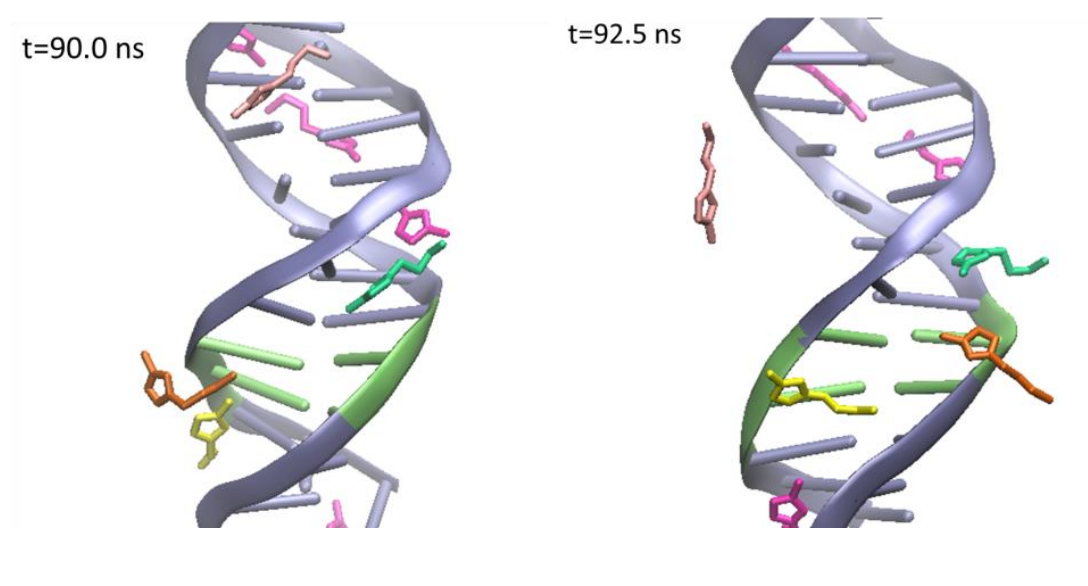

$t=94.9 \mathrm{~ns}$
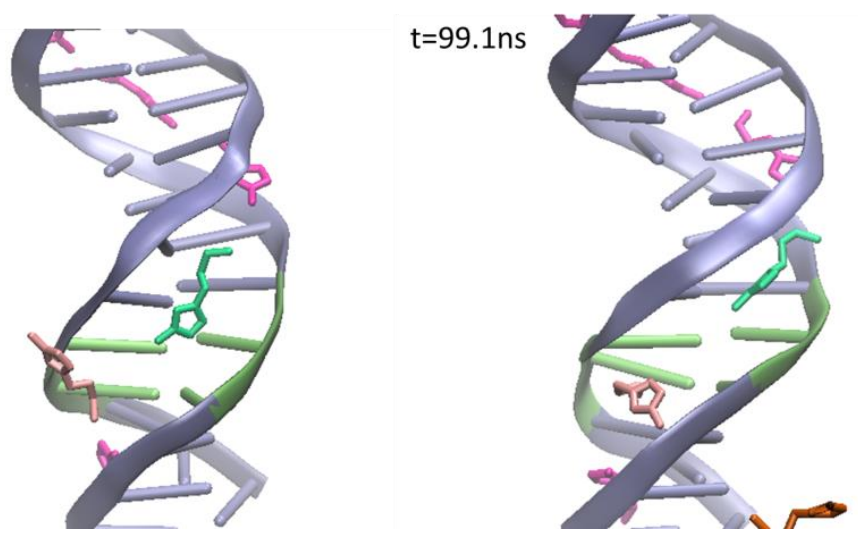

Figure 2-11 Visualization of IL repeat 1 at different time. At $92.5 \mathrm{~ns}$, the dissociation was found followed by the rebinding and shift of green cation at $94.9 \mathrm{~ns}$.

\subsection{Conclusion}

In summary, in this chapter we conducted MD simulation to study the DNA conformational change in IL. The coordinations of atoms were first converted to collective internal parameters, followed by PCA which showed the distribution pattern for different environments. Our PCA revealed the subtle conformational change in IL and further related such change to binding of cations. We further noticed C-G and its proximal region showed more affinity to cations and the conformation of 
such region also suffered more change upon binding. We further noticed C-G and its proximal region showed more affinity to cations and the conformation of such region also suffered more change upon binding. The principal component analysis of internal parameter vectors was proved to be a powerful way to explore such subtle conformational change and can expand its application to broader circumstances, such as epigenetic change or mismatch et al. 


\section{Reference}

(1) Rich, A.; Nordheim, A.; Wang, A. H. J. The Chemistry and Biology of Left-Handed Z-DNA. Апnи Rev Biochem 1984, 53, 791.

(2) Krzyzaniak, A.; Salanski, P.; Jurczak, J.; Barciszewski, J. B-Z DNA Reversible Conformation Changes Effected by High-Pressure. Febs Lett 1991, 279 (1), 1.

(3) Herbert, A. G.; Rich, A. A Method to Identify and Characterize Z-DNA Binding-Proteins Using a Linear Oligodeoxynucleotide. Nucleic Acids Res 1993, 21 (11), 2669.

(4) Herbert, A.; Alfken, J.; Kim, Y. G.; Mian, I. S.; Nishikura, K.; Rich, A. A Z-DNA binding domain present in the human editing enzyme, double-stranded RNA adenosine deaminase. $P$ Natl Acad Sci USA 1997, 94 (16), 8421.

(5) DiMaio, F.; Yu, X.; Rensen, E.; Krupovic, M.; Prangishvili, D.; Egelman, E. H. A virus that infects a hyperthermophile encapsidates A-form DNA. Science 2015, 348 (6237), 914.

(6) Ke, F. Y.; Luu, Y. K.; Hadjiargyrou, M.; Liang, D. H. Characterizing DNA Condensation and Conformational Changes in Organic Solvents. Plos One 2010, 5 (10).

(7) Georgiades, S. N.; Abd Karim, N. H.; Suntharalingam, K.; Vilar, R. Interaction of Metal Complexes with G-Quadruplex DNA. Angew Chem Int Edit 2010, 49 (24), 4020.

(8) Noy, A.; Perez, A.; Laughton, C. A.; Orozco, M. Theoretical study of large conformational transitions in DNA: the B <-> A conformational change in water and ethanol/water. Nucleic Acids Res 2007, 35 (10), 3330.

(9) Moradi, M.; Babin, V.; Roland, C.; Sagui, C. Reaction path ensemble of the B-Z-DNA transition: a comprehensive atomistic study. Nucleic Acids Res 2013, 41 (1), 33.

(10) Vrikkis, R. M.; Fraser, K. J.; Fujita, K.; MacFarlane, D. R.; Elliott, G. D. Biocompatible Ionic Liquids: A New Approach for Stabilizing Proteins in Liquid Formulation. J Biomech Eng-T Asme 2009, 131 (7).

(11) Armand, M.; Endres, F.; MacFarlane, D. R.; Ohno, H.; Scrosati, B. Ionic-liquid materials for the electrochemical challenges of the future. Nat Mater 2009, 8 (8), 621.

(12) Vijayaraghavan, R.; Izgorodin, A.; Ganesh, V.; Surianarayanan, M.; MacFarlane, D. R. Long-Term Structural and Chemical Stability of DNA in Hydrated Ionic Liquids. Angew Chem Int Edit 2010, 49 (9), 1631.

(13) Chandran, A.; Ghoshdastidar, D.; Senapati, S. Groove Binding Mechanism of Ionic Liquids: A Key Factor in Long-Term Stability of DNA in Hydrated Ionic Liquids? J Am Chem Soc 2012, 134 (50), 20330.

(14) Tateishi-Karimata, H.; Sugimoto, N. A-T Base Pairs are More Stable Than G-C Base Pairs in a Hydrated Ionic Liquid. Angew Chem Int Edit 2012, 51 (6), 1416.

(15) Jumbri, K.; Rahman, M. B. A.; Abdulmalek, E.; Ahmad, H.; Micaelo, N. M. An insight into structure and stability of DNA in ionic liquids from molecular dynamics simulation and experimental studies. Phys Chem Chem Phys 2014, 16 (27), 14036.

(16) Nakano, M.; Tateishi-Karimata, H.; Tanaka, S.; Sugimoto, N. Choline Ion Interactions with DNA Atoms Explain Unique Stabilization of A-T Base Pairs in DNA Duplexes: A Microscopic View. J Phys Chem B 2014, 118 (2), 379.

(17) Xuan Shuguang, Meng, Z. Y., Wu Xiangyang, Wong Jiun-Ru, Devi Gitali, Edwin Yeow Kok Lee, Shao Fangwei Efficient DNA-mediated Electron Transport in Ionic Liquids. ACS Sustainable Chemistry and Engineering 2016. 
(18) Lu, X. J.; Olson, W. K. 3DNA: a software package for the analysis, rebuilding and visualization of three-dimensional nucleic acid structures. Nucleic Acids Res 2003, 31 (17), 5108.

(19) Lu, X. J.; Olson, W. K. 3DNA: a versatile, integrated software system for the analysis, rebuilding and visualization of three-dimensional nucleic-acid structures. Nat Protoc 2008, 3 (7), 1213.

(20) Zgarbova, M.; Sponer, J.; Otyepka, M.; Cheatham, T. E.; Galindo-Murillo, R.; Jurecka, P. Refinement of the Sugar-Phosphate Backbone Torsion Beta for AMBER Force Fields Improves the Description of Z- and B-DNA. J Chem Theory Comput 2015, 11 (12), 5723.

(21) Krepl, M.; Zgarbova, M.; Stadlbauer, P.; Otyepka, M.; Banas, P.; Koca, J.; Cheatham, T. E.; Jurecka, P.; Sponer, J. Reference Simulations of Noncanonical Nucleic Acids with Different chi Variants of the AMBER Force Field: Quadruplex DNA, Quadruplex RNA, and Z-DNA. $J$ Chem Theory Comput 2012, 8 (7), 2506.

(22) Zgarbova, M.; Luque, F. J.; Sponer, J.; Cheatham, T. E.; Otyepka, M.; Jurecka, P. Toward Improved Description of DNA Backbone: Revisiting Epsilon and Zeta Torsion Force Field Parameters. J Chem Theory Comput 2013, 9 (5), 2339.

(23) Jorgensen, W. L.; Chandrasekhar, J.; Madura, J. D.; Impey, R. W.; Klein, M. L. Comparison of Simple Potential Functions for Simulating Liquid Water. J Chem Phys 1983, 79 (2), 926.

(24) Wu, X. P.; Liu, Z. P.; Huang, S. P.; Wang, W. C. Molecular dynamics simulation of room-temperature ionic liquid mixture of [bmim][BF4] and acetonitrile by a refined force field. Phys Chem Chem Phys 2005, 7 (14), 2771.

(25) Bussi, G.; Donadio, D.; Parrinello, M. Canonical sampling through velocity rescaling. J Chem Phys 2007, 126 (1).

(26) Berendsen, H. J. C.; Postma, J. P. M.; Vangunsteren, W. F.; Dinola, A.; Haak, J. R. Molecular-Dynamics with Coupling to an External Bath. J Chem Phys 1984, 81 (8), 3684.

(27) Berendsen, H. J. C.; Vanderspoel, D.; Vandrunen, R. Gromacs - a Message-Passing Parallel Molecular-Dynamics Implementation. Comput Phys Commun 1995, 91 (1-3), 43.

(28) Lindahl, E.; Hess, B.; van der Spoel, D. GROMACS 3.0: a package for molecular simulation and trajectory analysis. J Mol Model 2001, 7 (8), 306.

(29) Van der Spoel, D.; Lindahl, E.; Hess, B.; Groenhof, G.; Mark, A. E.; Berendsen, H. J. C. GROMACS: Fast, flexible, and free. J Comput Chem 2005, 26 (16), 1701.

(30) Hess, B.; Kutzner, C.; van der Spoel, D.; Lindahl, E. GROMACS 4: Algorithms for highly efficient, load-balanced, and scalable molecular simulation. J Chem Theory Comput 2008, 4 (3), 435 .

(31) Hess, B.; Bekker, H.; Berendsen, H. J. C.; Fraaije, J. G. E. M. LINCS: A linear constraint solver for molecular simulations. J Comput Chem 1997, 18 (12), 1463.

(32) Lu, X. J.; ElHassan, M. A.; Hunter, C. A. Structure and conformation of helical nucleic acids: Analysis program (SCHNAaP). J Mol Biol 1997, 273 (3), 668.

(33) Humphrey, W.; Dalke, A.; Schulten, K. VMD: Visual molecular dynamics. J Mol Graph Model 1996, 14 (1), 33.

(34) Numata, J.; Wan, M.; Knapp, E. W. Conformational entropy of biomolecules: Beyond the quasi-harmonic approximation. Genome Inform Ser 2007, 18, 192. 


\section{Chapter 3}

A Theoretical Study of DNA-Mediated Charge

Transport in Ionic Liquid 


\subsection{Introduction}

\subsubsection{The experimental studies of DNA CT}

The hypothesis of DNA as a potential charge transport media was proposed as early as $1960 \mathrm{~s}^{1}$. But it was until 1993 that the first experimental evidence was reported $^{2}$. In their pioneering work, Barton et al. introduced $\left[\mathrm{Ru}(\mathrm{phen})_{2} \mathrm{dppz}\right]^{2+}$ as an electron hole donor and $\left[\mathrm{Rh}(\mathrm{phi})_{2} \text { phen }\right]^{3+}$ as an acceptor. Both the donor and acceptor can intercalate into the duplex via a flexible covalent linkage onto DNA strand. By measuring the quenching of donor fluorescence, the efficiency of CT can be estimated indirectly. Since then, tremendous studies have been reported: Lewis et al. used stilbene as a donor to construct hairpin to study the CT and their results showed the distance dependence of $\mathrm{CT}^{3}$. Majima et al. reported the same distance dependence phenomenon using pyrene as donor ${ }^{4}$. Besides, Schuster et al. used anthraquinone as donor and proposed a polaron-like CT machenism ${ }^{5}$. Different from previous photochemical method to study CT, Tao et al. employed DNA covalently linked to two gold electrodes and investigated CT by measuring the conductance ${ }^{6}$. To date, the importance of DNA CT and its role in many pivotal biological processes, such as DNA repair, DNA replication, transcription and protein translation ${ }^{7}$, has been recognized widely.

\subsubsection{CT through DNA stacked base pairs}

Because of the similarity between the stacking base pair and graphene layers, the DNA's conductivity has been hypothesized and experimentally confirmed as mentioned above. It's widely accepted that the electron or electron hole migrates 
through the $\pi-\pi$ stacking of the neighboring base pairs and the disturbance of such stacking will highly dump the CT efficiency. For example, Z-form DNA, which shows a more widely spaced base pair stacking, gives less CT efficiency; on the other hand, A-form DNA with a less disturbed structure will facilitate CT rate $^{8}$. Besides, other perturbance, such as mismatch or bulge, also inhibits CT to a large extent ${ }^{9,10}$.

\subsubsection{Basic mechanism for CT}

In the study of CT through DNA, the electron or electron hole was usually introduced by irradiation toward donor, which will then obtain or lose one electron; because of the driving force from donor, the extra electron or hole will subsequently migrate through the stacking DNA pairs until it was trapped by the acceptor. When electron was introduced, it will migrate through the LUMO orbitals of the base sites. While for hole transport, the HOMO orbitals of the bases will be employed to act as the pathway, and the electron will migrate from accepter to donor, as figure 3-1 shows.

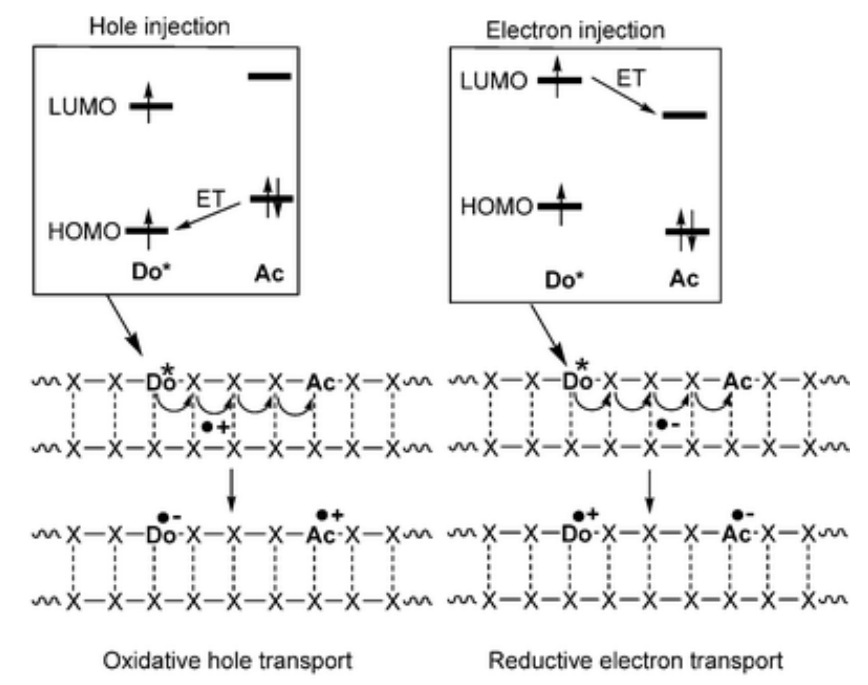

Figure 3-1 The scheme of electron transport and hole transport. Graph was taken from ref 11 
Thereafter the roles of different bases will be discussed with regard to hole transfer. In general, from the oxidative potential of different bases (figure 3-2), purines are easy to undergo hole transport and pyrimidines prefer electron transfer. Guanine has the lowest oxidative potential, so it can give out the electron most easily, furthermore consecutive guanine, such as GG doublet and GGG triplet, usually act as the natural hole trap ${ }^{12}$. All of the nucleobases, including adenine, guanine, thymine and cytosines, participate in conducting electron holes. With cyclopropylamine modified nucleobases, the transient hole occupancies during migration were observed for all nucleobases, except thymines ${ }^{13-15}$.
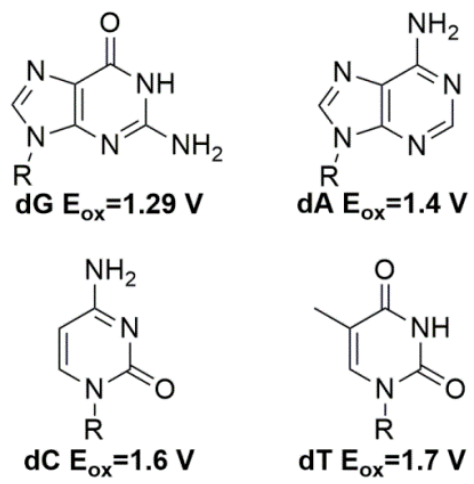

Figure 3-2 Structure and oxidation potential versus NHE of four bases.

According to whether the relaxation of charge is allowed in these bridge sites, the mechanism of DNA CT can be divided into two main categories generally: coherent superexchange and incoherent hopping (figure 3-3). In superexchange, the electron or hole is not allowed to relax upon the bridge site, as a result, the CT rate shows an exponential decay dependence upon distance:

$$
k_{C T}=k_{0} \exp (-\beta R)
$$


where $\mathrm{R}$ is the distance between donor and acceptor, $\beta$ the decay factor and $\mathrm{k}_{0}$ the pre-exponential constant. Experimental value of $\beta$ ranges from $0.2 \AA^{-1}$ to $1.42 \AA^{-12,16}$. On the other hand, for hopping the CT rate shows shallower distance dependence since the electron or hole is allowed to relax and jump from site to site incoherently. The rate value is thus around linearly dependent on the number of bridge sites:

$$
k_{C T}=P^{*} N^{-\eta}
$$

where $\mathrm{N}$ is the number of bridge sites and $\mathrm{P}$ and $\eta$ are the constants. $\eta$ value lies within $1 \sim 2^{17}$. Though the mechanism under specific condition is still under debate, it's now widely accepted that CT through DNA is a competing and compromise process between two main mechanisms: superexchange tunneling dominates short-sequence CT while for longer sequence (usually more than 4 bases) the incoherent hopping will instead overweight ${ }^{18}$.

The above mentioned model gives a quite simple formula for CT. However, they both ignore the possible delocalization of extra charge, which stabilize the system. Alternatively, in conformational gated domain CT theory, the charge was supposed to delocalize within several bases (a domain), and the propagation of the charge was gated by the fluctuation of conformation, when the conformation reached to certain optimal status, the charge can migrate simultaneously into next domain, otherwise the charge will stay at the original position ${ }^{19}$. 

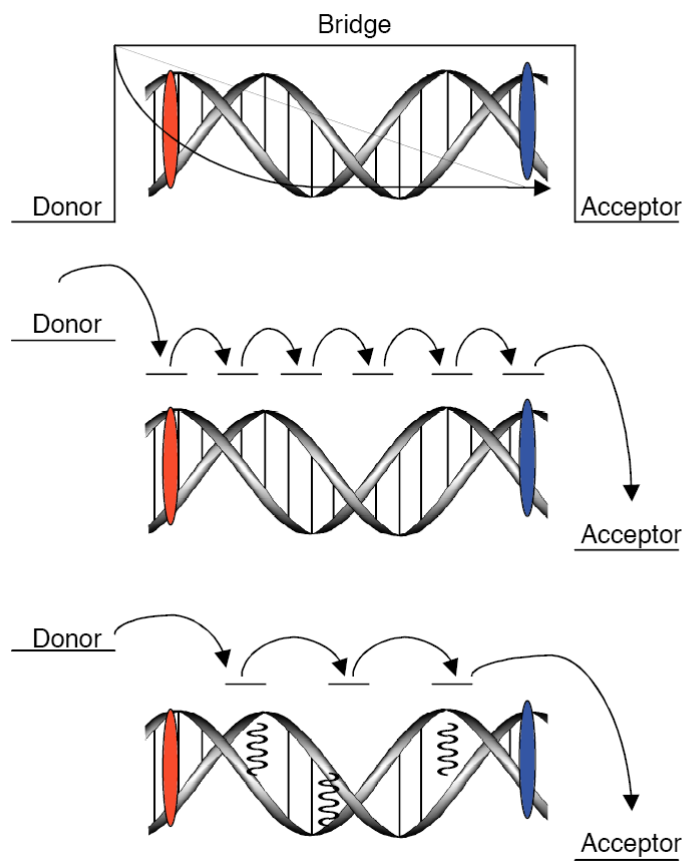

Figure 3-3 Schematic representation of 3 possible mechanisms. Top : super exchange; central: hopping; bottom: domain transport. Graph was taken from ref 20

Furthermore, the external environment may also contribute to the CT. Thus, a polaron like hopping mechanism was proposed (figure 3-4), which supposed the appearance of the extra charge will change the conformation of the neighboring bases accordingly, as well as the arrangement of proximal solvent molecules, to relieve the charge deficiency. The size of polaron was dependent on the balance between the degree of structural distortion and stabilization of the charge. The polaron can hop to next polaron with the help of leaving and joining of neighbouring bases (phonon) ${ }^{5}$. Such model overcame the shortcomings of the previous static models by explaining some important phenomenon of DNA CT, such as the decrease of the CT barrier and involvement of all kinds of bases ${ }^{21}$. 


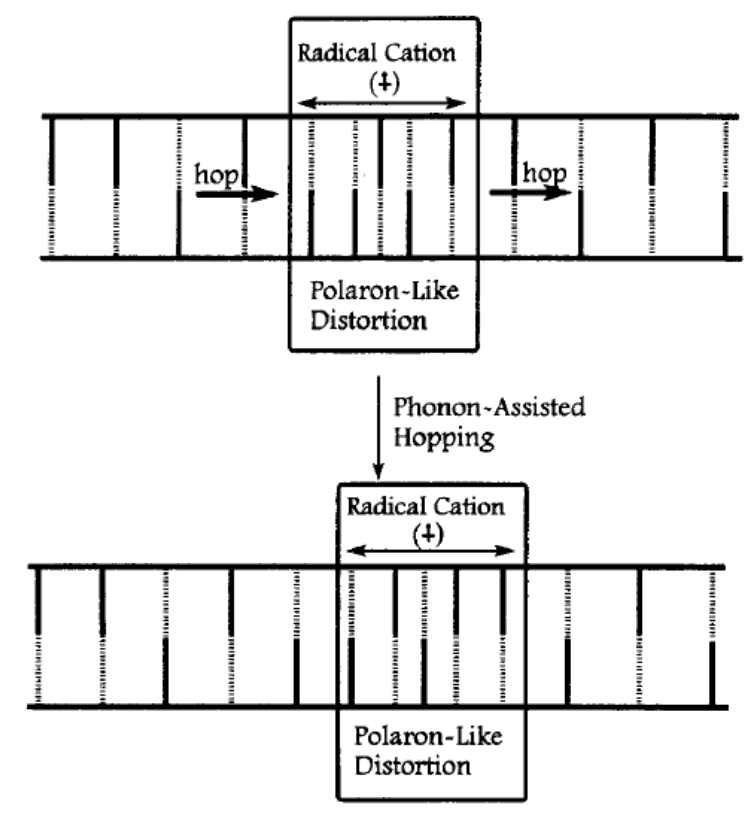

Figure 3-4 Schematic representation of phonon-assisted polaron hopping mechanism.

The graph was taken from ref 5

Up to this end, it seems that these mechanisms based on experimental studies were controversial. Therefore, it would be beneficial to employ some theoretical methods to investigate such problem and provide a more valid background for DNA CT.

\subsubsection{The theoretical studies of DNA CT}

Since the pioneering work of Marcus in $1956^{22}$, the formula to estimate CT rate has been explored exhaustively by classical, semi-classical and quantum-mechanical $\operatorname{methods}^{23,24}$.

\subsubsection{The initial picture of CT: a Marcus scheme}

Suppose an electron transfer reaction within a redox complex:

$$
D^{-} A \rightarrow D A^{-}
$$


where D and A denote electron donor and acceptor, respectively. In the beginning the electron resides on the donor and the energy stays in its minimum, with the thermal fluctuation of the redox complex, the free energy of the system $\mathrm{D}^{-} \mathrm{A}$ may increase to approach the crossover area, where the free energy between $\mathrm{D}^{-} \mathrm{A}$ and $\mathrm{DA}^{-}$is small enough for electron to hop from donor to acceptor, as shown in figure 3-5. Here the reaction coordinates are indicated by diabatic energy gap, as suggested by Zusman ${ }^{25}$ and Warshel ${ }^{26}$ :

$$
\Delta E\left(\boldsymbol{R}^{N}\right)=E_{b}\left(\boldsymbol{R}^{N}\right)-E_{a}\left(\boldsymbol{R}^{N}\right)
$$

where $E_{a}$ and $E_{b}$ denote the diabatic energy of $D^{-} A$ and $D^{-}$respectively. $\lambda$ refers to the reorganization energy, i.e. the energy required to rearrange the redox complex and solvent molecules to allow the reaction to happen. Besides, we define the red curve as adiabatic state and blue dashed line diabatic state. We noticed that there's no crossover between two adiabatic curves, that's because the under adiabatic approximation the Hamiltonian of two states ( $\mathrm{a}$ and $\mathrm{b}$ ) will be diagonalized:

$$
\begin{gathered}
\mathcal{H}=\left(\begin{array}{cc}
E_{a} & H_{a b} \\
H_{b a} & E_{b}
\end{array}\right) \\
E_{0 / 1}\left(\boldsymbol{R}^{N}\right)=\frac{E_{a}\left(\boldsymbol{R}^{N}\right)+E_{b}\left(\boldsymbol{R}^{N}\right)}{2} \pm \frac{1}{2} \sqrt{\Delta E^{2}\left(\boldsymbol{R}^{N}\right)+4\left|H_{a b}\right|^{2}\left(\boldsymbol{R}^{N}\right)}
\end{gathered}
$$

where $\mathrm{H}_{\mathrm{ab}}$ denotes the electronic coupling between state $\mathrm{a}$ and $\mathrm{b}$ and $\mathrm{E}_{0 / 1}$ the energy of adiabatic states. 


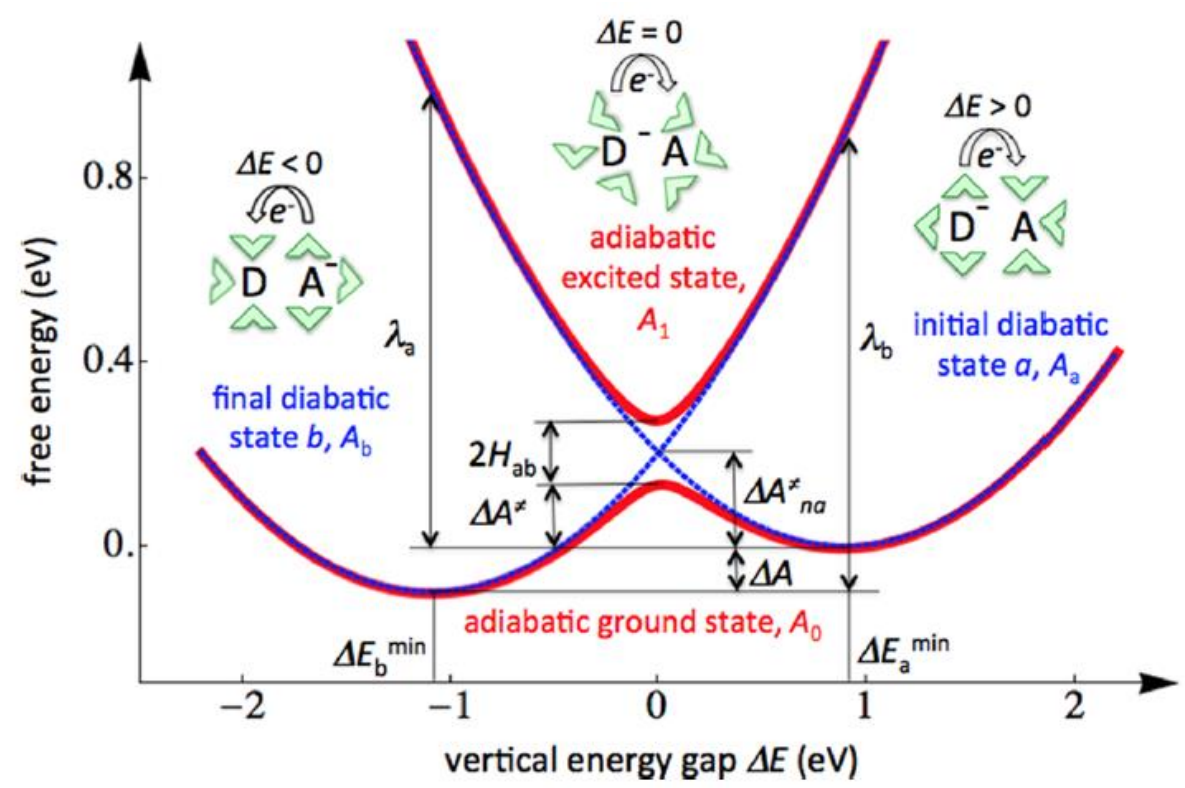

Figure 3-5 A charge transport picture under Marcus scheme. The red lines denote adiabatic free energy curve and blue dotted lines denote diabatic free energy curve. Graph was taken from ref 27

From the above picture we can derive the CT rate formula. Before that, some approximations need to be made.

The energy land scape of biomolecule is smooth and entropic effect is more obvious. Thus the free energy is used instead of pure enthalpy, To estimate the free energy directly is difficult, however, under normal situations we can just suppose a Gaussian distribution of free energy for a given diabatic state. Therefore the free energy curves of diabatic states show a parabolic dependence on diabatic energy ${ }^{27}$ :

$$
\begin{gathered}
A_{a}=\frac{1}{4 \lambda}(\Delta E-(\Delta A+\lambda))^{2} \\
A_{b}=\frac{1}{4 \lambda}(\Delta E-(\Delta A-\lambda))^{2}+\Delta A
\end{gathered}
$$

where 


$$
\begin{gathered}
\lambda \equiv \lambda_{a}=\lambda_{b} \\
\Delta E_{M} \equiv\langle\Delta E\rangle_{M}=\Delta E_{M}^{\text {min }} \\
\lambda=\left(\Delta E_{a}-\Delta E_{b}\right) / 2 \\
\Delta A=\left(\Delta E_{a}+\Delta E_{b}\right) / 2
\end{gathered}
$$

and the activation energy for adiabatic curve reads as:

$$
\Delta A_{n a}^{\neq}=\frac{(\lambda+\Delta A)^{2}}{4 \lambda}
$$

The activation energy difference between diabatic states and adiabatic states is:

$$
\Delta=\left\langle\left|H_{a b}\right|^{2}\right\rangle_{T S}^{1 / 2}+\frac{\lambda+\Delta A-\sqrt{(\lambda+\Delta A)^{2}+4\left\langle\left|H_{a b}\right|^{2}\right\rangle_{a}}}{2}
$$

If we further regard the nuclei as a classical particle, together with transition state theory, we can finally derive the CT rate as ${ }^{27}$ :

$$
k_{c}=\kappa_{e l} v_{n} \exp \left(-\frac{\Delta A^{\neq}}{k_{B} T}\right)
$$

where $v_{\mathrm{n}}$ is the effective nuclear frequency along the reaction coordinate, and $\kappa_{\mathrm{el}}$ is the electronic transmission coefficient:

$$
\begin{gathered}
\kappa_{e l}= \begin{cases}\frac{2 P_{L Z}}{1+P_{L Z}} & \text { if } \Delta A \geq-\lambda \\
2 P_{L Z}\left(1-P_{L Z}\right) & \text { if } \Delta A<-\lambda\end{cases} \\
P_{L Z}=1-\exp (-2 \pi \gamma) \\
2 \pi \gamma=\frac{\pi^{3 / 2}\left\langle\left|H_{a b}\right|^{2}\right\rangle_{T S}}{h v_{n} \sqrt{\lambda k_{B} T}}
\end{gathered}
$$

where $P_{L Z}$ is so called Laudau-Zener transition probability which gives the probability of the electron staying around crossover region.

Up to this end the basic mechanism of CT is illustrated. From the above 
discussion we can see that the CT rate is mainly dependent on the coupling $H_{a b}$ as well as the diabatic energy $\Delta E_{M}$. Although above-mentioned formulas give a comprehensive expression of CT formulas, it should be pointed out they are based on several approximations and have limitation to some extent.

\subsubsection{CT over multistate}

The single-step CT occurs when donor and acceptor are close enough to allow enough electronic coupling to form. However, for CT within biological molecules which happens at a longer distance, more than one CT state will be employed. According to whether the relaxation is allowed along bridge sites, 3 types of model will be reviewed and the schemes for them are shown in figure 3-6.
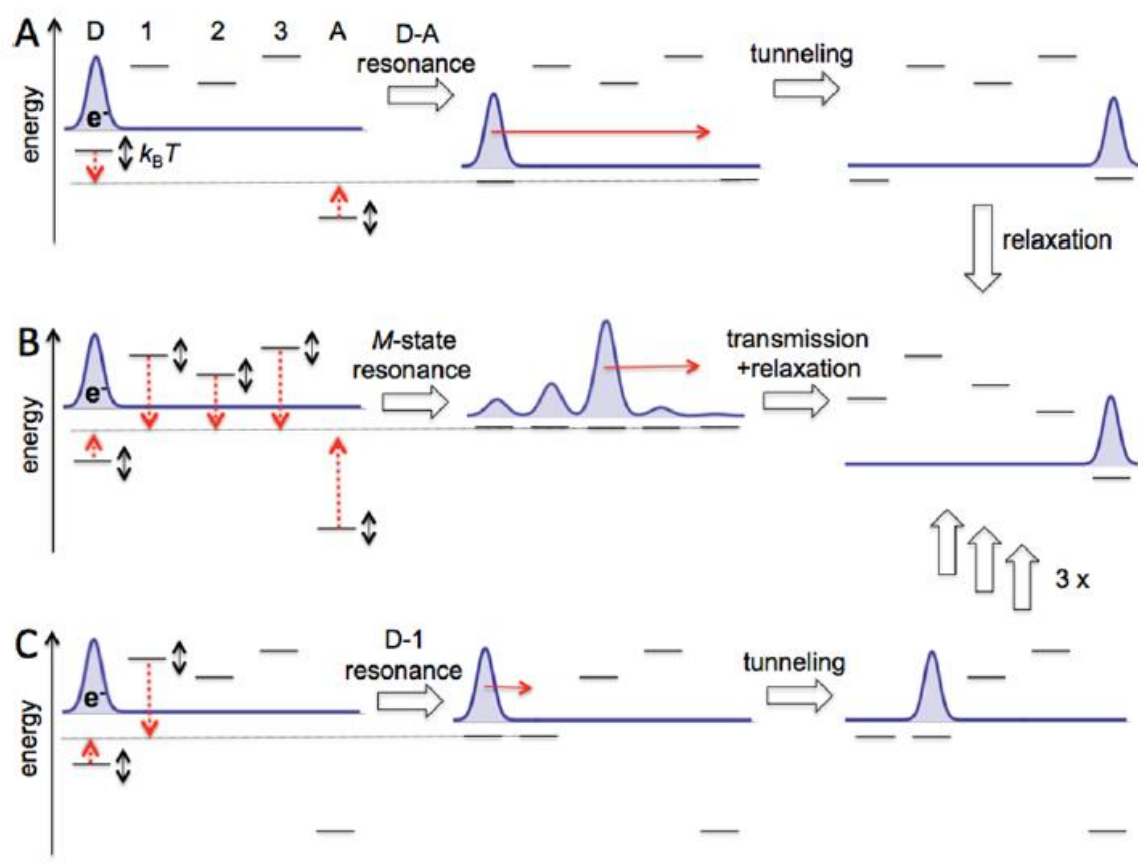

Figure 3-6 Schematic representation of 3 multistate CT model. A: Superexchange model; B: Flickering resonance model; C: Hopping model. The graph was taken from ref 27 


\subsection{Superexchange}

In superexchange model the bridge site provides merely the pathway for electron to migrate from donor to acceptor, i.e. the electron is not allowed to relax along the pathway. When the energy of bridge site is higher enough than the one of donor/bridge sites, the following equations hold ${ }^{27}$ :

$$
\begin{gathered}
H_{a b} \approx H_{D 1} G_{1 N}^{B} H_{N A} \\
G_{1 N}^{B}=\left(\prod_{i=1}^{N-1} \frac{H_{i i+1}^{B}}{E_{D / A}-H_{i i}^{B}}\right) \frac{1}{E_{D / A}-H_{N N}^{B}}
\end{gathered}
$$

where $G_{i j}^{B}$ are the matrix elements of the Green's function of the bridge sites. If we further assume $H_{D 1}=H_{i i+1}^{B}=H_{N A}$ and all $H_{i i}^{B}$ remain the same, then we have:

$$
\begin{gathered}
\left|H_{a b}\right|^{2}=V^{2} \exp [-\beta(R-\Delta R)] \\
\beta=\frac{2}{\Delta R} \ln \left|\frac{\Delta E_{D / A}-\Delta E_{B}}{V}\right| \\
V=\left\langle\left|H_{i i+1}^{B}\right|^{2}\right\rangle^{1 / 2} \\
k_{S E}=\frac{2 \pi}{\hbar} \frac{V^{2}}{\sqrt{4 \pi \lambda k_{B} T}} \exp [-\beta(R-\Delta R)] \exp \left[-\frac{(\Delta A+\lambda)}{4 \lambda k_{B} T}\right]
\end{gathered}
$$

in which $\Delta R$ is the distance between neighboring sites and $R$ the distance between donor and acceptor. The $E_{D / A}$ and $H_{i i}^{B}$ are replaced by electron removal energy $\Delta E_{D / A}$ and $\Delta E_{B}$. As eq 3.24 shows, the rate decreases exponentially as the distance between donor and acceptor increases, so superexchange usually applies to relatively short distance.

\subsection{Flickering resonance}

In flickering resonance the bridge sites are allowed to fluctuate to achieve 
resonance, when the electron can migrate directly from donor to acceptor. The CT rate is then dependent on the probability to achieve the resonance ${ }^{27}$ :

$$
\begin{array}{cc}
P(M)=\int_{-\infty}^{+\infty} \mathrm{d} \Delta E_{1} \rho_{1}\left(\Delta E_{1}\right) \int_{\Delta E_{1}-V}^{\Delta E_{1}+V} \mathrm{~d} \Delta E_{2} \rho_{2}\left(\Delta E_{2}\right) \cdots & \\
\int_{\max \left\{\Delta E_{1}, \Delta E_{2}, \cdots, \Delta E_{M-1}\right\}-V}^{\min \left\{\Delta E_{1}, \Delta E_{2}, \cdots, \Delta E_{M-1}\right\}+V} \mathrm{~d} \Delta E_{M} \rho_{M}\left(\Delta E_{M}\right) & \text { Eq } 3.25 \\
k_{F R}^{\max }=\frac{4 \sqrt{2}}{\hbar R / \Delta R} \frac{V^{2}}{\sqrt{4 \pi \lambda k_{B} T}} \exp [-\Theta(R-\Delta R)] \exp \left[-\frac{\left(\Delta E_{D / A}-\Delta E_{B}\right)^{2}}{2 \lambda k_{B} T}\right] \exp \left[-\frac{\left(\Delta E_{D}-\Delta E_{A}\right)^{2}}{4 \lambda k_{B} T}\right] & \text { Eq } 3.26 \\
\Theta=\frac{1}{\Delta R} \ln \left(\sqrt{\left.\frac{\pi}{2} \frac{\sigma_{E}}{V}\right)}\right. & \text { Eq } 3.27 \\
\lambda=\frac{\sigma_{E}^{2}}{2 k_{B} T} & \text { Eq } 3.28
\end{array}
$$

where $\Delta \mathrm{E}_{\mathrm{D} / \mathrm{A}}=\left(\Delta \mathrm{E}_{\mathrm{D}}+\Delta \mathrm{E}_{\mathrm{A}}\right) / 2, \sigma_{\mathrm{E}}$ is the Gaussian width of the on-site energy. The meaning of each term can be illustrated more clearly in figure 3-7. The flickering resonance model is less distance dependent than superexchange and thus can be used for longer distance CT.

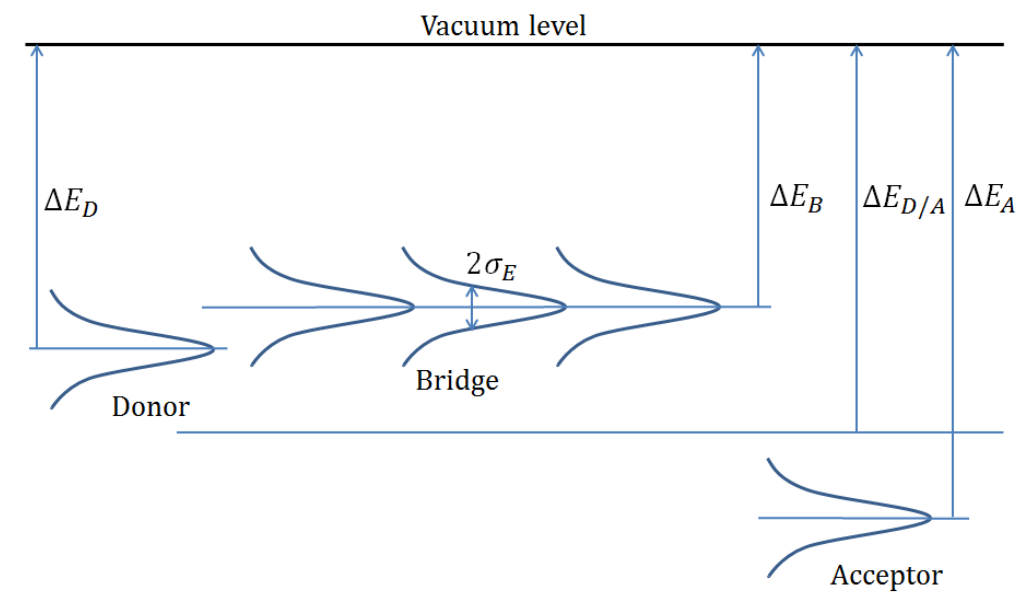

Figure 3-7 Definition of energetic quantities for ET in a simple CT system. The thermal fluctuation is represented by Gaussian distribution. 


\subsection{Hopping model}

For longer range CT, even resonance can hardly be achieved. Thus incoherent and discrete hopping transport will dominate. The idea is to allow charge to stay at each bridge site and each step is reversible, when the charge populations of the bridge sites are not significantly large, according to reaction dynamics we have ${ }^{27,28}$ :

$$
\begin{aligned}
& \dot{p_{D}}=-k_{1 D} p_{D}+k_{D 1} p_{1} \\
& \dot{p_{1}}=k_{1 D} p_{D}-\left(k_{D 1}+k_{21}\right) p_{1}+k_{12} p_{2} \\
& p_{M}=k_{M M-1} p_{M-1}-\left(k_{M-1 M}+k_{A M}\right) p_{M}+k_{M A} p_{A} \\
& \dot{p_{A}}=-k_{A M} p_{M}+k_{M A} p_{A}
\end{aligned}
$$

Now we further assume $\mathrm{p}_{\mathrm{D}}=$ const, $\mathrm{p}_{\mathrm{A}}=0$ and all the rate constant of bridge sites $\mathrm{k}_{\mathrm{ij}}$ are the same, the rate formula can be written as:

$$
k_{\text {hop }}=\frac{\exp \left[-\Delta A_{B D} /\left(k_{B} T\right)\right]}{1 / k_{A N}+1 / k_{D 2}+[(R / \Delta R)-2] / k_{B B}}
$$

Different from superexchange and flickering resonance, the hopping rate shows linear dependence on the CT distance.

\subsubsection{Recent theoretical studies of DNA CT}

The above-mentioned theories are all based on Marcus-like picture, which assumes the canonical free energy curve and localization of charge in donor and acceptor. However such pre-conceived model over-simplifies the CT picture and cannot describe the CT picture on the fly. The drawbacks of such model become 
obvious when the coupling between donor and acceptor is much larger than the reorganization energy and the $\mathrm{CT}$ time scale is much faster than the vibrational frequency of the molecules, which are usually true for DNA CT. The quantum filling method and non-adiabatic molecular dynamics are both based the Schrodinger equation to propagate the wave function of the charge and as a result can avoid the incorrect estimation due to pre-conceived picture.

\subsection{Quantum filling}

In quantum filling model, the wave function of the charge is propagated by Liouville equation $^{29}$ :

$$
\frac{\mathrm{d} \rho(t)}{\mathrm{d} t}=-\frac{i}{\hbar}[\mathcal{H}, \rho(t)]
$$

where $\mathcal{H}$ consists of 3 components:

$$
\mathcal{H}=\mathcal{H}_{S}+\mathcal{H}_{B}+\mathcal{H}_{S B}
$$

in which $\mathcal{H}_{\mathrm{S}}, \mathcal{H}_{\mathrm{B}}$ and $\mathcal{H}_{\mathrm{SB}}$ stand for the Hamiltonian of DNA, the bath and interaction between DNA and the bath, respectively. Once these three parts are defined properly, the movement of the wave function can be simulated. This model allow the relaxation and decoherence among bridge sites, as well as the coupling between DNA and the bath, thus provided a quite refined model of DNA CT. Recently the same group proposed a modified model employing Lindblad equation ${ }^{30}$, which proved to be computationally less expensive. 


\subsection{Non-adiabatic molecular dynamics}

The drawback of quantum filling is the structure of DNA remains fixed during the simulation. Non-aidabatic molecular dynamics ${ }^{31,32}$ can propagate the wave function of the charge and the DNA structure simultaneously. Different from normal MD method, such hybrid QM/MM method divide the system into two parts: the base part where the charge will migrate through will be treated quantum mechanically, and the rest part will be treated molecular mechanically. The MM calculation and QM calculation will be conducted alternately: the whole system will be propagated molecular mechanically first, thereafter a QM calculation will apply to base part, from which the atomic charge of QM atoms will be renewed after each QM step, as figure 3-8 shows. The QM and MM part are then coupled. Because the charge of the base part is not consistent anymore, such MD method is therefore non-adiabatic. It should be pointed out such method provided a robust method to simulate DNA CT on the fly, while its high computational demand requires much approximated QM method. 


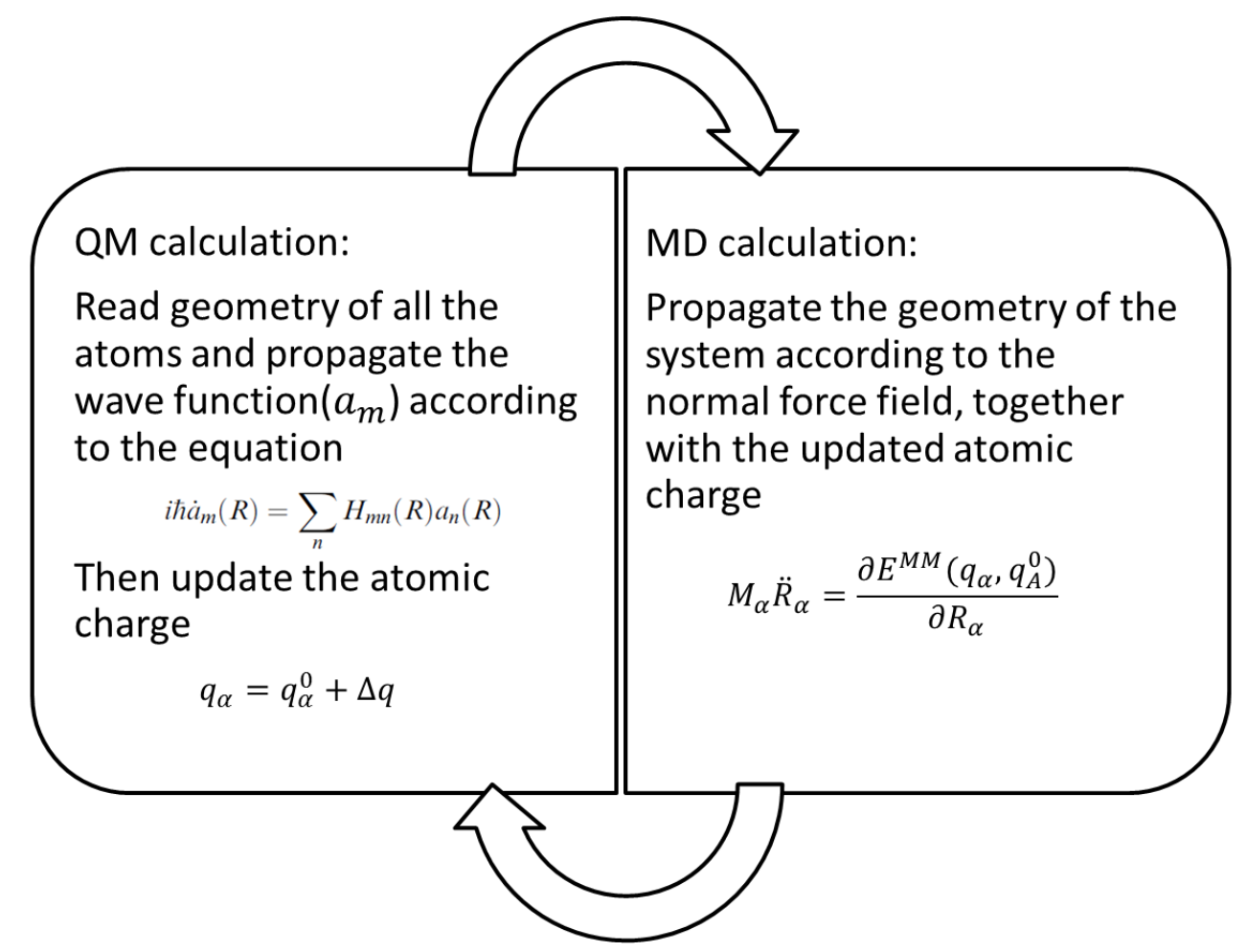

Figure 3-8 Schematic representation of non-adiabatic molecular dynamics.

\subsubsection{Research motivation: DNA CT in ionic liquid}

Though the stacking between bases makes the CT through DNA feasible, the sensitivity of coupling between bases towards structure fluctuation makes the CT far from optimum. Up to date many approaches have been proposed to facilitate DNA CT, such as use of modified bases ${ }^{33,34}$, extending conjugated area ${ }^{35}$ and application of external magnetic field ${ }^{36}$.

As mentioned above, the solvent plays a critical role in DNA CT. Theoretical study has shown that both the mean value and deviation were significantly changed in water instead of in vacuum ${ }^{37}$. More importantly, the increase of deviation made it possible for on-site energy of neighboring bases to overlap to facilitate CT. Lewis et al. reported the facilitation of DNA CT in chloroform, which suggested the solvent may tune the CT rate by stabilizing on-site energy ${ }^{38}$. 
In last chapter the advantage of IL as a biocompatible solvent was introduced. According to existing researches, our group was interested in the ability of IL to facilitate DNA CT. The experimental scheme was illustrated in figure 3-9. Under photo irradiation, a charge hole will be introduced into the DNA system which will migrate through DNA until trapped by the acceptor. By HPLC analysis the decomposition yield of acceptor can be determined and used as a mark for CT rate. Our previous data showed that the DNA in $[\mathrm{BMIM}]\left[\mathrm{BF}_{4}\right]$ possessed inverse distance dependence $^{39}$, as shown in figure 3-9. Besides, time-resolved fluorescence decay shows better stacking was achieved in ionic liquid ${ }^{39}$. Such experimental findings left inspiration as well as questions. Due to the lack of explanation with regard to the reason to cause such CT facilitation phenomenon, computational method is required to investigate such problem, with the hope to provide more microscopic description of DNA conformation in different solution environments. In last chapter we have already employed principal component analysis (PCA) to treat MD trajectory, the results demonstrated the subtle conformational change of DNA in IL; in this chapter, QM/MM method was used to compute the onsite energy and coupling between base pairs, followed by calculation of $\mathrm{CT}$ rate using flickering resonance model and hopping model.

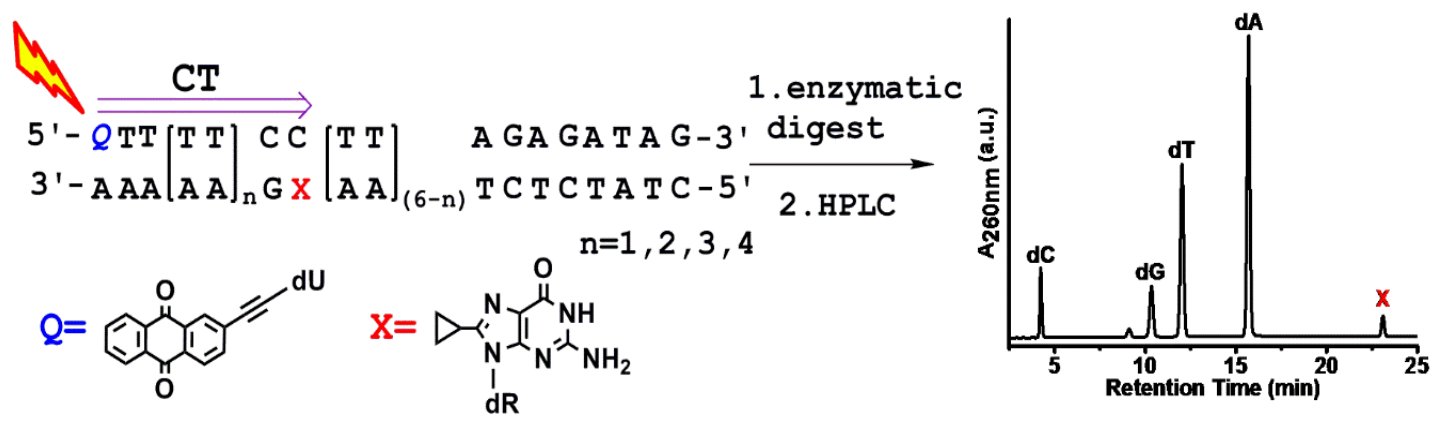




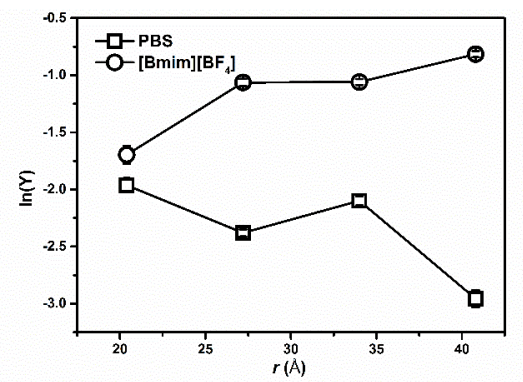

Figure 3-9 Upper: experimental scheme to investigate CT rate; lower: the distance dependence of CT yield in aqueous buffer and ionic liquid. The graph were taken from ref 39

\subsection{Methods}

\subsubsection{QM calculation employing fragment orbital density functional theory (FO-DFTB) method}

The QM part of DNA was illustrated in figure 3-10. Only the base part was calculated quantum mechanically.

$$
\begin{array}{llllllllllllllllllllll}
\mathbf{5} & , & - & \mathbf{T} & \mathbf{T} & \mathbf{T} & \mathbf{T} & \mathbf{T} & \mathbf{T} & \mathbf{T} & \mathbf{T} & \mathbf{T} & \mathbf{T} & \mathbf{T} & \mathbf{C} & \mathbf{C} & \mathbf{T} & \mathbf{T} & \mathbf{A} & - & \mathbf{3} \\
\mathbf{3} & , & - & \mathbf{A} & \mathrm{A} & \mathbf{A} & \mathbf{A} & \mathbf{A} & \mathbf{A} & \mathbf{A} & \mathbf{A} & \mathbf{A} & \mathbf{A} & \mathbf{A} & \mathrm{G} & \mathrm{G} & \mathbf{A} & \mathbf{A} & \mathbf{T} & - & \mathbf{5}
\end{array},
$$

Figure 3-10 The sequence used for QM/MM calculation. All colorful bases were treated quantum mechanically, among them yellow one denoted donor and green one denoted acceptor. Among them are bridge sites. The black bases are treated molecular mechanically.

The required terms for CT calculation, i.e. the onsite energy of each base and electronic coupling between bases, were computed using FO-DFTB method ${ }^{40}$ according to last $10 \mathrm{~ns}$ MD trajectory. For each frame, we can have a Hamiltonian matrix composed of the onsite energy and electronic coupling. Here the basic idea of 
FO-DFTB will be introduced.

First of all, the fragment orbital, which will localize within base part was built up from atomic orbital:

$$
\varphi_{m}^{i}=\sum_{\mu} c_{\mu}^{i m} \eta_{\mu}
$$

here $\eta_{\mu}$ denotes atomic orbital, $\varphi_{\mathrm{m}}^{\mathrm{i}}$ the fragment orbital and $c_{\mu}^{\mathrm{im}}$ the corresponding coefficient which were determined under SCC-DFTB scheme. Such approximated DFTB method is much faster than normal DFT method because the overlap integral and Hamiltonian are computed and stored beforehand:

$$
\begin{gathered}
S_{\mu \nu}=\left\langle\eta_{\mu} \mid \eta_{\nu}\right\rangle \\
H_{\mu \nu}^{0}=\left\langle\eta_{\mu}\left|\widehat{H}\left[\rho_{\alpha}+\rho_{\beta}\right]\right| \eta_{\nu}\right\rangle
\end{gathered}
$$

and the SCF method was applied to minimize the total energy:

$$
\begin{gathered}
E_{S C C}=\sum_{i \mu \nu} c_{\mu}^{i} c_{\nu}^{i} H_{\mu \nu}^{0}+\frac{1}{2} \gamma_{\alpha \beta} \Delta q_{\alpha} \Delta q_{\beta}+\frac{1}{2} U_{\alpha \beta} \\
H_{\mu \nu}=H_{\mu \nu}^{0}+\frac{1}{2} S_{\mu \nu}^{\alpha \beta}\left(\sum_{\delta} \Delta q_{\delta}\left(\gamma_{\alpha \delta}+\gamma_{\beta \delta}\right)+\sum_{A} Q_{A}\left(\frac{1}{r_{A \alpha}}+\frac{1}{r_{A \beta}}\right)\right) \text { Eq } 3.36
\end{gathered}
$$

In which $\alpha, \beta, \delta$ are the index of atoms and $\mathrm{A}$ is the index of MM atom. It should be pointed here the effect of solvent were taken into consideration explicitly. For charge hole transport, the HOMO of each base will be selected out to form the Hamiltonian matrix of base part:

$$
T_{m n}=\left\langle\varphi_{m}^{H O}|\widehat{H}| \varphi_{n}^{H O}\right\rangle=\sum_{\mu} \sum_{\nu} c_{\mu}^{m} c_{\nu}^{n} H_{\mu \nu}
$$

where $m, n$ is the index of CT site. Here, instead of $H_{\mu \nu}$, a new term, $T_{m n}$ is used to 
distinguish it from Hamiltonian matrix of basis set.

\subsubsection{Calculation of CT rate using different multistate model}

With onsite energy and electronic coupling in hand, the CT rate can be estimated using different model. Because of the long distance between donor and acceptor, the superexchange model was not taken into consideration. The other two model, flickering resonance and hopping, are both employed. The formulas were listed in section 3.1.4.2.2 and 3.1.4.2.3. Here we took the last $10 \mathrm{~ns}$ trajectory to analyze, according to the experimental measured rate scale ${ }^{41}$. The mean on-site energy, coupling $V=\left\langle\left|H_{a b}\right|^{2}\right\rangle^{1 / 2}$ and the $\sigma_{\mathrm{E}}$ were calculated from all the frames. For hopping model $\Delta A_{B D}$ was supposed to be 0 and rate between two neighboring sites was calculated using Marcus equation ${ }^{24}$ :

$$
k=\frac{2 \pi}{\hbar}\left|H_{D A}\right|^{2} \frac{1}{\sqrt{4 \pi \lambda k_{B} T}} \exp \left[-\frac{\left(\Delta E_{D}-\Delta E_{A}\right)^{2}}{4 \lambda k_{B} T}\right]
$$

where $H_{D A}$ is the coupling and $\Delta E_{D}, \Delta E_{A}$ is on-site energy for each donor and acceptor pair.

\subsection{Results and discussion}

\subsubsection{IL stabilized onsite energy of DNA base}

With QM/MM calculation, the onsite energy and electronic coupling of $10 \mathrm{~ns}$ trajectory can be obtained. The average onsite energy for each repeat both in water and IL were plotted first, as shown in figure 3-11. In water all 3 repeats gave consistent pattern with the average onsite energy of adenine to be around $5.8 \mathrm{eV}$ and 
that of guanine to be around $5.4 \mathrm{eV}$. The appearance of IL lowers the onsite energy for both kinds of bases by $\sim 0.4 \mathrm{eV}$. Besides, the values were highly fluctuated. Such fluctuation may be due to the close contact of cation. The standard deviation $\sigma_{\mathrm{E}}$ and averaged coupling were calculated and shown in table 3-1. The $\sigma_{\mathrm{E}}$ in hydrated IL $(\sim 0.296 \mathrm{eV})$ is a little less than the ones in pure water $(\sim 0.305 \mathrm{eV})$. The value was consistent with other group's result ${ }^{42}$.

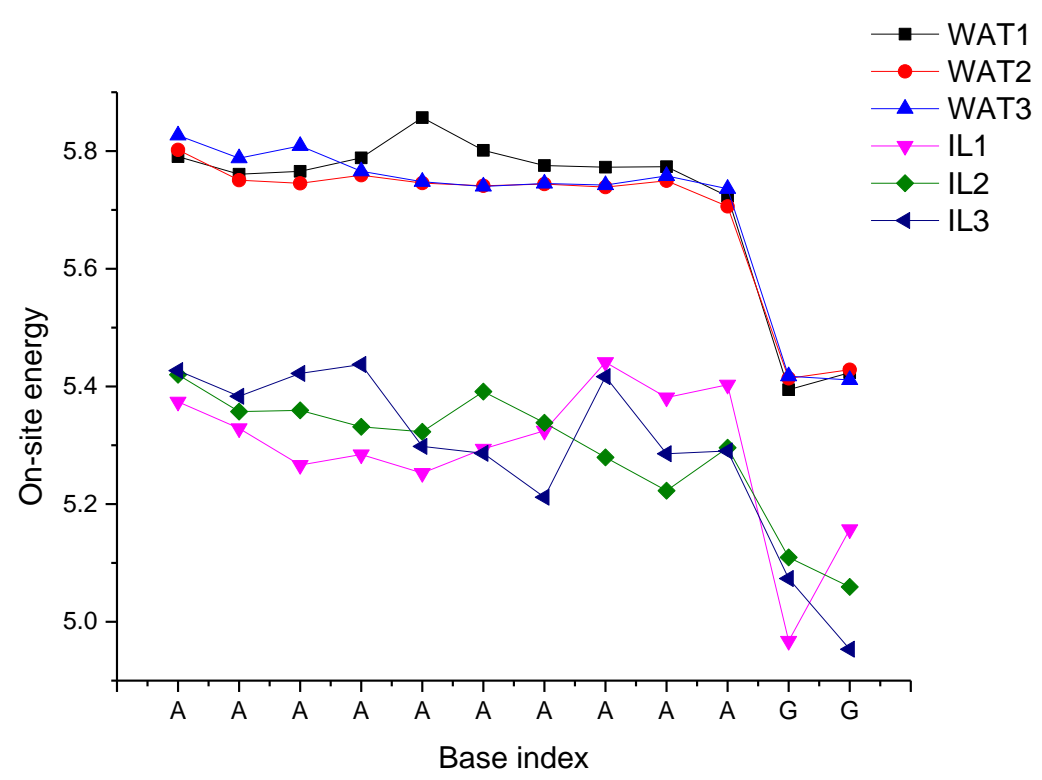

Figure 3-11 Plot of onsite energy of all CT sites over last $10 \mathrm{~ns}$.

Table 3-1 The time-average of coupling and standard deviation of on-site energy over last $10 \mathrm{~ns}$ in IL and water.

\begin{tabular}{lllll}
\hline No. & $\mathbf{V}_{I L}(\mathrm{eV})$ & $\mathrm{V}_{\text {Water }}(\mathrm{eV})$ & $\boldsymbol{\sigma}_{\mathrm{IL}}(\mathrm{eV})$ & $\boldsymbol{\sigma}_{\text {Water }}(\mathrm{eV})$ \\
\hline $\mathbf{1}$ & $0.10 \pm 0.02$ & $0.10 \pm 0.02$ & 0.298 & 0.308 \\
& & & & \\
$\mathbf{2}$ & $0.10 \pm 0.02$ & $0.09 \pm 0.02$ & 0.296 & 0.305
\end{tabular}




\subsubsection{IL can facilitate CT rate under FR model}

The CT rate was first estimated using flickering resonance model. Admittedly, long range CT over 9 bridge sites cannot occur without relaxation, however, if we ignore the contribution of incoherent process, such model will still shed a light upon the mechanism of CT. From previous study a 10 ns period was enough to allow one CT to happen ${ }^{29}$, thus we took the last 10ns second trajectory from each simulation, from which the $\Delta E_{X}, X=D, A, D / A, \sigma_{\mathrm{E}}$ and $\mathrm{V}$ were first computed as a time average, which can be further used to calculate the rate according to eq 3.39. The result was shown in table 3-2. Interestingly, the rate in IL showed rather fluctuation from 3.03/ns to $6.77 / \mathrm{ns}$, and the rate in water gave a less fluctuated value around $1.6 / \mathrm{ns}$. Overall the IL showed speedup effect towards CT rate by more than twice. Furthermore, such facilitation was mainly due to the increase of coupling between bases. To illustrate this we calculated the terms required to obtain final rate. It was found that the difference in $\Theta$ was enlarged by factor $(R-\Delta R)$ and thus made a main contribution to the difference of the final value. Compared with $\Theta(R-\Delta R)$, the contribution from on-site energy difference was trivial. The computational results were consistent with our experimental fluorescence decay result which suggested a better stacking between base pairs $^{39}$ 
We further plotted the coupling vector, $V$ for the last $10 \mathrm{~ns}$ in 3 IL repeats to illustrate the relationship between coupling and dynamic conformational motion. Among all the 5000 frames, the first 100 frames with largest $V$ (CT facilitation state) were filtered out and marked as blue triangle in figure 3-12. In spite of the large fluctuation (standard deviation up to $\sim 0.02 \mathrm{eV}$ ), IL repeat $2 / 3$ showed no obvious increment of coupling value over the period. However high $V$ values were apparently concentrated to the time period after $7 \mathrm{~ns}$ for IL repeat 1 , which indicated that the CT facilitation states were much more available over this period. Interestingly, this time period of high coupling effects were synchronized with the binding of IL cations and the establishment of stable conformation (region 4 in section 2.4.4). Therefore we attribute such enhancement of coupling energy to the dynamic motion of base pairs: when the nucleobases underwent large conformational change, it had no time to adjust base conformation to facilitate CT; only after duplex DNA reached a stable conformation upon the optimal binding of IL cations, DNA was allowed enough relax time at this confined conformation to further fine tune the alignment of nucleobase pairs along helical axis to achieve higher base pair coupling for efficient CT.

Apart from FR model, we also employed hopping model based on the same data to estimate the CT rate of last 10 ns trajectory. As shown in table 3-3, the result (up to $10^{12} / \mathrm{s}$ ) was much larger than those from FR model, which was due to the incoherent property of hopping model. Interestingly, under such model the discrepancy between IL and water disappeared, because the subtle increase of coupling in CT between neighboring sites was not large enough to facilitate final rate significantly. The failure 
of hopping model to estimate CT rate difference between water and IL indicated the mechanism of CT over long range was still coherent, while FR model also underestimated the CT rate compared with experimental results ${ }^{29}$ because of the total neglecting of relaxation of bridge sites. Therefore the CT over long range may be an intermediate between coherent and incoherent process: the charge can migrate over several bridge sites simultaneously when resonance was achieved by fluctuation, while relaxation was allowed among bridge sites to increase the possibility of CT. Between the two processes the FR may be still dominating.

Table 3-2 CT rates and related terms in different environments (above: IL, below: water) under FR model

\begin{tabular}{|c|c|c|c|c|c|}
\hline \multirow{3}{*}{ Repeat No. } & \multirow{3}{*}{$\frac{V^{2}}{\sqrt{4 \pi \lambda k_{B} T}}$} & \multicolumn{4}{|c|}{ IL } \\
\hline & & \multirow{2}{*}{$\Theta(R-\Delta R)$} & \multirow{2}{*}{$\frac{\left(\Delta E_{D / A}-\Delta E_{B}\right)^{2}}{2 \lambda k_{B} T}$} & \multirow{2}{*}{$\frac{\left(\Delta E_{D}-\Delta E_{A}\right)^{2}}{4 \lambda k_{B} T}$} & \multirow{2}{*}{$k^{F R}\left(n s^{-1}\right)$} \\
\hline & & & & & \\
\hline 1 & -4.58 & 11.52 & 0.144 & 0.464 & $4.74 \times 10^{-2}$ \\
\hline 2 & -4.58 & 11.48 & 0.019 & 0.274 & $6.77 \times 10^{-2}$ \\
\hline \multirow[t]{2}{*}{3} & -4.71 & 12.05 & 0.043 & 0.358 & $3.03 \times 10^{-2}$ \\
\hline & & \multicolumn{4}{|c|}{ WAT } \\
\hline Repeat No. & $\frac{V^{2}}{\sqrt{4 \pi \lambda k_{B} T}}$ & $\Theta(R-\Delta R)$ & $\frac{\left(\Delta E_{D / A}-\Delta E_{B}\right)^{2}}{2 \lambda k_{B} T}$ & $\frac{\left(\Delta E_{D}-\Delta E_{A}\right)^{2}}{4 \lambda k_{B} T}$ & $k^{F R}\left(n s^{-1}\right)$ \\
\hline 1 & -4.75 & 12.40 & 0.185 & 0.414 & $1.67 \times 10^{-2}$ \\
\hline 2 & -4.78 & 12.50 & 0.097 & 0.405 & $1.63 \times 10^{-2}$ \\
\hline 3 & -4.78 & 12.42 & 0.010 & 0.445 & $1.53 \times 10^{-2}$ \\
\hline
\end{tabular}



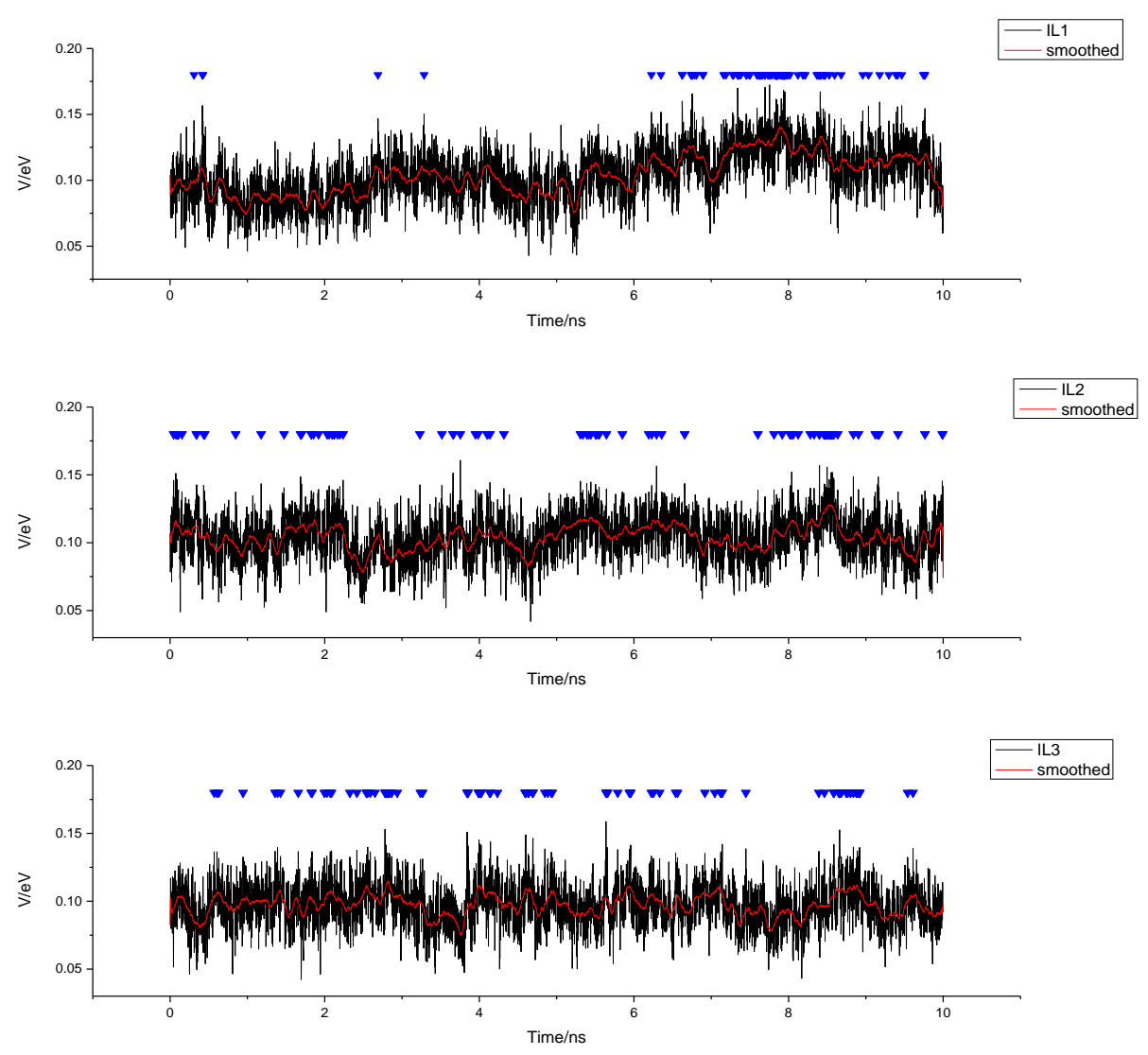

Figure 3-12 Plot of electronic coupling for $3 \mathrm{IL}$ repeats over last $10 \mathrm{~ns}$. The red line was smoothed curve and blue triangles denoted the times corresponding to largest to largest coupling frames.

Table 3-3 CT rates over last $10 \mathrm{~ns}$ in IL and water computed under hopping model

\begin{tabular}{ccc}
\hline \multirow{2}{*}{ Repeat No. } & $\mathbf{I L}$ & WAT \\
\cline { 2 - 3 } & $k^{\text {Hopping }}\left(\mathrm{ns}^{-1}\right)$ & $k^{\text {Hopping }}\left(\mathrm{ns}^{-1}\right)$ \\
\hline $\mathbf{1}$ & $1.504 \times 10^{3}$ & $1.548 \times 10^{3}$ \\
$\mathbf{2}$ & $1.554 \times 10^{3}$ & $1.321 \times 10^{3}$ \\
$\mathbf{3}$ & $1.272 \times 10^{3}$ & $1.337 \times 10^{3}$ \\
\hline
\end{tabular}

\subsection{Conclusion}

In this chapter, hybrid QM/MM method was employed to compute onsite energy and coupling which could be used to estimate long range CT rate under FR and hopping model. Under FR model the facilitation of IL to CT rate can be estimated 
correctly, however such facilitation was no longer hold under hopping model. Besides the discrepancy between FR and hopping models suggested a partially coherent mechanism which allowed relaxation to happen. Compared with existing methods, our method only require MD trajectory for computing CT rate so the chronological conformational change as well as solvent effect can be taken into consideration. At the same time, such scheme only required simple post QM/MM calculation after MD simulation and therefore can accelerate the computational speed and expand to longer time scale.

In conclusion, our study sorts out the relationship among the binding modes, the conformation dynamics and the CT rate. Such correlations between the three may provide deeper insight into the CT of DNA. 


\section{Reference}

(1) Eley, D. D.; Spivey, D. I. Semiconductivity of Organic Substances .9. Nucleic Acid in Dry State. T Faraday Soc 1962, 58 (470), 411.

(2) Murphy, C. J.; Arkin, M. R.; Jenkins, Y.; Ghatlia, N. D.; Bossmann, S. H.; Turro, N. J.; Barton, J. K. Long-Range Photoinduced Electron-Transfer through a DNA Helix. Science 1993, 262 (5136), 1025 .

(3) Lewis, F. D.; Wu, T. F.; Zhang, Y. F.; Letsinger, R. L.; Greenfield, S. R.; Wasielewski, M. R. Distance-dependent electron transfer in DNA hairpins. Science 1997, 277 (5326), 673.

(4) Kawai, K.; Takada, T.; Tojo, S.; Ichinose, N.; Majima, T. Observation of hole transfer through DNA by monitoring the transient absorption of pyrene radical cation. J Am Chem Soc 2001, 123 (50), 12688.

(5) Henderson, P. T.; Jones, D.; Hampikian, G.; Kan, Y. Z.; Schuster, G. B. Long-distance charge transport in duplex DNA: The phonon-assisted polaron-like hopping mechanism. P Natl Acad Sci USA 1999, 96 (15), 8353.

(6) He, H. X.; Li, C. Z.; Tao, N. J. Direct measurement of the conductance of single conducting polymer nanowire and its application in biological nanosensor. Abstr Pap Am Chem S 2000, 220, U262.

(7) Grodick, M. A.; Muren, N. B.; Barton, J. K. DNA Charge Transport within the Cell. Biochemistry-Us 2015, 54 (4), 962.

(8) Boon, E. M.; Barton, J. K. DNA Electrochemistry as a probe of base pair stacking in A-, B-, and Z-form DNA. Bioconjugate Chem 2003, 14 (6), 1140.

(9) Boon, E. M.; Ceres, D. M.; Drummond, T. G.; Hill, M. G.; Barton, J. K. Mutation detection by electrocatalysis at DNA-modified electrodes. Nat Biotechnol 2000, 18 (10), 1096.

(10) Delaney, S.; Yoo, J.; Stemp, E. D. A.; Barton, J. K. Charge equilibration between two distinct sites in double helical DNA. P Natl Acad Sci USA 2004, 101 (29), 10511.

(11) Wagenknecht, H. A. Electron transfer processes in DNA: mechanisms, biological relevance and applications in DNA analytics. Nat Prod Rep 2006, 23 (6), 973.

(12) Saito, I.; Nakamura, T.; Nakatani, K.; Yoshioka, Y.; Yamaguchi, K.; Sugiyama, H. Mapping of the hot spots for DNA damage by one-electron oxidation: Efficacy of GG doublets and GGG triplets as a trap in long-range hole migration. J Am Chem Soc 1998, 120 (48), 12686.

(13) Nakatani, K.; Dohno, C.; Saito, I. Design of a hole-trapping nucleobase: Termination of DNA-mediated hole transport at N-2-cyclopropyldeoxyguanosine. J Am Chem Soc 2001, 123 (39), 9681.

(14) Shao, F. W.; O'Neill, M. A.; Barton, J. K. Long-range oxidative damage to cytosines in duplex DNA. P Natl Acad Sci USA 2004, 101 (52), 17914.

(15) Dohno, C.; Ogawa, A.; Nakatani, K.; Saito, I. Hole trapping at N-6-cyclopropyideoxyadenosine suggests a direct contribution of adenine bases to hole transport through DNA. J Am Chem Soc 2003, 125 (34), 10154.

(16) Fukui, K.; Tanaka, K. Distance dependence of photoinduced electron transfer in DNA. Angew Chem Int Edit 1998, 37 (1-2), 158.

(17) Jortner, J.; Bixon, M.; Langenbacher, T.; Michel-Beyerle, M. E. Charge transfer and transport in DNA. P Natl Acad Sci USA 1998, 95 (22), 12759.

(18) Lewis, F. D.; Zhu, H. H.; Daublain, P.; Fiebig, T.; Raytchev, M.; Wang, Q.; Shafirovich, V. 
Crossover from superexchange to hopping as the mechanism for photoinduced charge transfer in DNA hairpin conjugates. J Am Chem Soc 2006, 128 (3), 791.

(19) O'Neill, M. A.; Becker, H. C.; Wan, C. Z.; Barton, J. K.; Zewail, A. H. Ultrafast dynamics in DNA-mediated electron transfer: Base gating and the role of temperature. Angew Chem Int Edit 2003, 42 (47), 5896.

(20) Boon, E. M.; Barton, J. K. Charge transport in DNA. Curr Opin Struc Biol 2002, 12 (3), 320.

(21) Genereux, J. C.; Barton, J. K. Mechanisms for DNA Charge Transport. Chem Rev 2010, 110 (3), 1642 .

(22) Marcus, R. A. On the Theory of Oxidation-Reduction Reactions Involving Electron Transfer .1. J Chem Phys 1956, 24 (5), 966.

(23) Newton, M. D.; Sutin, N. Electron-Transfer Reactions in Condensed Phases. Annu Rev Phys Chem 1984, 35, 437.

(24) Marcus, R. A.; Sutin, N. Electron Transfers in Chemistry and Biology. Biochim Biophys Acta 1985, 811 (3), 265.

(25) Zusman, L. D. Outer-Sphere Electron-Transfer in Polar-Solvents. Chem Phys 1980, 49 (2), 295.

(26) Warshel, A. Dynamics of Reactions in Polar-Solvents - Semi-Classical Trajectory Studies of Electron-Transfer and Proton-Transfer Reactions. J Phys Chem-Us 1982, 86 (12), 2218.

(27) Blumberger, J. Recent Advances in the Theory and Molecular Simulation of Biological Electron Transfer Reactions. Chem Rev 2015, 115 (20), 11191.

(28) Nitzan, A. Chemical dynamics in condensed phases : relaxation, transfer, and reactions in condensed molecular systems; Oxford University Press: New York, 2006.

(29) Renaud, N.; Berlin, Y. A.; Lewis, F. D.; Ratner, M. A. Between Superexchange and Hopping: An Intermediate Charge-Transfer Mechanism in Poly(A)-Poly(T) DNA Hairpins. J Am Chem Soc 2013, 135 (10), 3953.

(30) Renaud, N.; Harris, M. A.; Singh, A. P.; Berlin, Y. A.; Ratner, M. A.; Wasielewski, M. R.; Lewis, F. D.; Grozema, F. C. Deep-hole transfer leads to ultrafast charge migration in DNA hairpins. Nat Chem 2016, 8 (11), 1015.

(31) Kubar, T.; Elstner, M. Coarse-Grained Time-Dependent Density Functional Simulation of Charge Transfer in Complex Systems: Application to Hole Transfer in DNA. J Phys Chem B 2010, 114 (34), 11221.

(32) Kubar, T.; Elstner, M. Efficient algorithms for the simulation of non-adiabatic electron transfer in complex molecular systems: application to DNA. Phys Chem Chem Phys 2013, 15 (16), 5794.

(33) Kawai, K.; Kodera, H.; Majima, T. Long-Range Charge Transfer through DNA by Replacing Adenine with Diaminopurine. J Am Chem Soc 2010, 132 (2), 627.

(34) Thazhathveetil, A. K.; Trifonov, A.; Wasielewski, M. R.; Lewis, F. D. Increasing the Speed Limit for Hole Transport in DNA. J Am Chem Soc 2011, 133 (30), 11485.

(35) Delaney, S.; Barton, J. K. Charge transport in DNA duplex/quadruplex conjugates. Biochemistry-Us 2003, 42 (48), 14159.

(36) Wong, J. R.; Lee, K. J.; Shu, J. J.; Shao, F. W. Magnetic Fields Facilitate DNA-Mediated Charge Transport. Biochemistry-Us 2015, 54 (21), 3392.

(37) Kubar, T.; Kleinekathofer, U.; Elstner, M. Solvent Fluctuations Drive the Hole Transfer in DNA: A Mixed Quantum-Classical Study. J Phys Chem B 2009, 113 (39), 13107. 
(38) Mishra, A. K.; Young, R. M.; Wasielewski, M. R.; Lewis, F. D. Wirelike Charge Transport Dynamics for DNA-Lipid Complexes in Chloroform. J Am Chem Soc 2014, 136 (44), 15792.

(39) Xuan Shuguang, Meng, Z. Y., Wu Xiangyang, Wong Jiun-Ru, Devi Gitali, Edwin Yeow Kok Lee, Shao Fangwei Efficient DNA-mediated Electron Transport in Ionic Liquids. ACS Sustainable Chemistry and Engineering 2016.

(40) Kitaura, K.; Ikeo, E.; Asada, T.; Nakano, T.; Uebayasi, M. Fragment molecular orbital method: an approximate computational method for large molecules. Chem Phys Lett 1999, 313 (3-4), 701.

(41) Lewis, F. D.; Zhu, H. H.; Daublain, P.; Cohen, B.; Wasielewski, M. R. Hole mobility in DNA A tracts. Angew Chem Int Edit 2006, 45 (47), 7982.

(42) Mario Wolter, M. E., Ulrich Kleinekathoefer, Tomas Kubar. Microsecond simulation of electron transfer in DNA: bottom-up parametrization of an efficient electron transfer model based on atomistic details. J Phys Chem B 2016. 


\section{Chapter 4}

Theoretical investigation of novel metal complex as G-quadruplex binder 


\subsection{Introduction}

G-quadruplex was one of the non-canonical structures of DNA which was formed by guanine rich nucleic acid. Since its important role in gene replication and transcription ${ }^{1}$, the study of G-quadruplex has drawn significant interests over years. Especially, the formation of G-quadruplex was found to highly relate to apoptosis of tumor cells ${ }^{2,3}$, as a result, the study of method to stabilize such secondary structure may provide promising method for cancer therapy. Here the structure of G-quadruplex will be first reviewed, followed by the introduction of metal complex as a G-quadruplex stabilizer.

\subsubsection{The structure and function of G-quadruplex}

G-quadruplex is the noncanonical DNA secondary structure formed by stacking of multi G-quartet layers. For each G-quartet layer, 4 guanine bases form a square plane by Hoogsteen bond. The guanine bases that form G-quartet can be from either single strand, two strand or 4 different strands, as shown in figure 4-1.

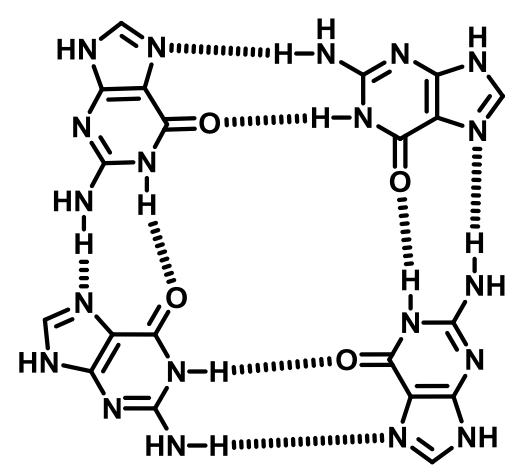




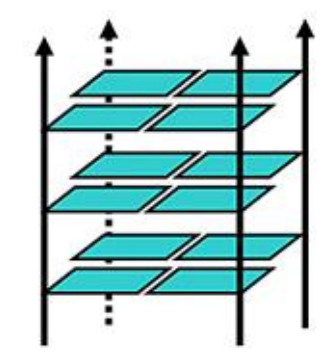

Intermolecular

B Tetrameric

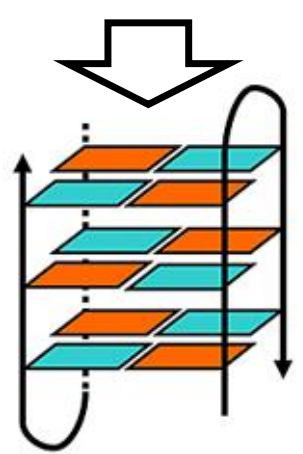

Dimeric

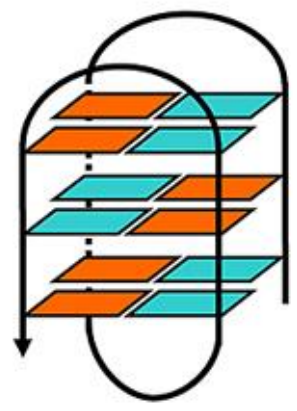

Intramolecular

Monomeric

Figure 4-1 Top: the structure of G-quartet; bottom: the G-quadruplexes formed by four strand, two strand or single strand DNA.

After the first report published in $1962^{4}$, up to date many different types of G-quadruplex has been found. Among them the monomeric G-quadruplex formed by human telomeric sequence which possesses tandem repeats of sequence 5'-TAACCC-3' has been provoked much interests. According to the direction of backbone, the monomeric G-quadruplex can further classified into antiparallel, parallel and hybrid form, as figure 4-2 shows.

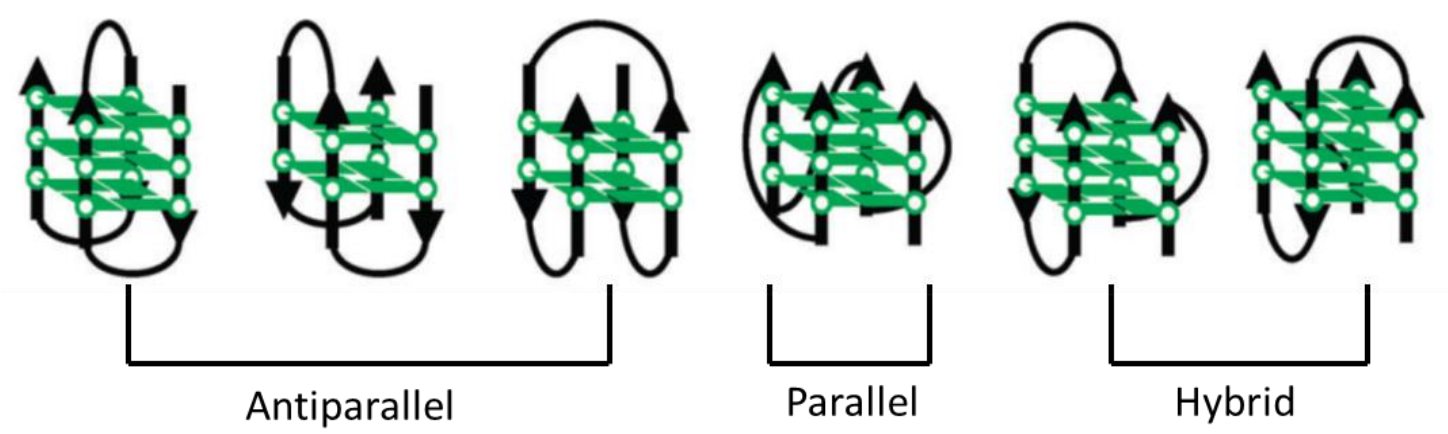

Figure 4-2 The different conformations of monomeric G-quadruplex, the graphs was taken from ref 5

The formation of telomeric G-quadruplex is highly related to telomerase activity. The tolemere will be shortened with every cell division, while in tumor cell the 
elongation reaction under the help of telomerase will make the cell immortal. However, the formation of G-quadruplex will inhibit the binding of telomerase and thus lead to cell apoptosis, as shown in figure 4-3.

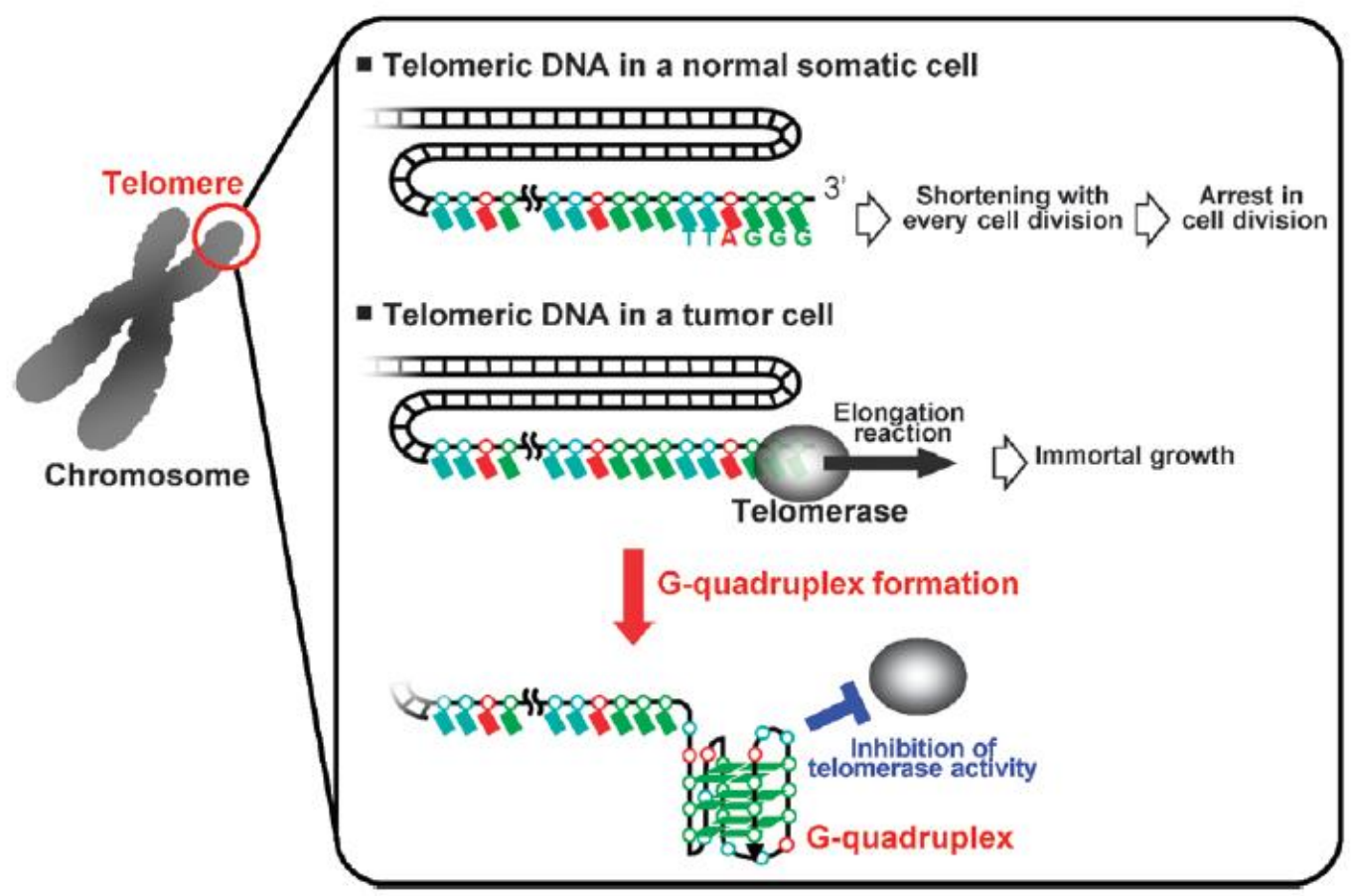

Figure 4-3 The formation of G-quadruplex may inhibit the binding of telomerase and thus avoid immortality of cancer cell. The graph was taken from ref 5

Besides telomeric G-quadruplex, c-myc G-quadruplex is also an important family of G-quadruplex which locates upstream of P1 promoter of c-myc. This 27-base G-rich sequence usually takes parallel conformation and is responsible for the block of transpcription of c-myc, which is over-expressed in tumor cells.

From the above discussion, G-quadruplex is like a switch: when it's formed, it inhibits the binding of corresponding protein and thus blocks the subsequent biological processes. Such regulatory effect is critical to prevent the immortalization 
of tumor cells. Therefore, to stabilize such structure selectively becomes extremely demanding. With such purpose, the planar structure interacting with G-quadruplex has been investigated extensively ${ }^{6-8}$.

\subsubsection{Metal complex as G-quadruplex stabilizer}

Metal complex can act as an ideal stabilizer for G-quadruplex because of its wide range of structural and electronic properties. In addition, their unique magnetic, optical and catalytic features make the exploration to employ them as novel G-quadruplex probes or catalysts possible.

As coordination complex, the constant coordinate geometry of metal center provides an easy way to introduce new ligand with different type of properties while at the same maintain the coordination geometry unchanged. By adjusting the size and geometry of ligands, it was feasible to enhance the selectivity of the complex. Besides, different metal centers possess different coordination geometry in nature, which makes it flexible to design new stabilizer according to the requirement.

Apart from the structural advantage, the metal center can withdraw electron from ligand, which will make the ligand electron-poor and the $\pi-\pi$ stacking interaction of ligand and G-quartet more stable. The positive metal center can act as the same role as alkali cation such as $\mathrm{Na}^{+}$and $\mathrm{K}^{+}$, it can locate above or between the centers of G-quartet and further stabilize the G-quadruplex.

The binding modes of metal complex towards G-quadruplex are also various: besides the normal external layer stacking, it can also intercalate between G-quartet 
layers or bind onto the groove region, as figure 4-4 shows. In the following some important metal complexes will be introduced.
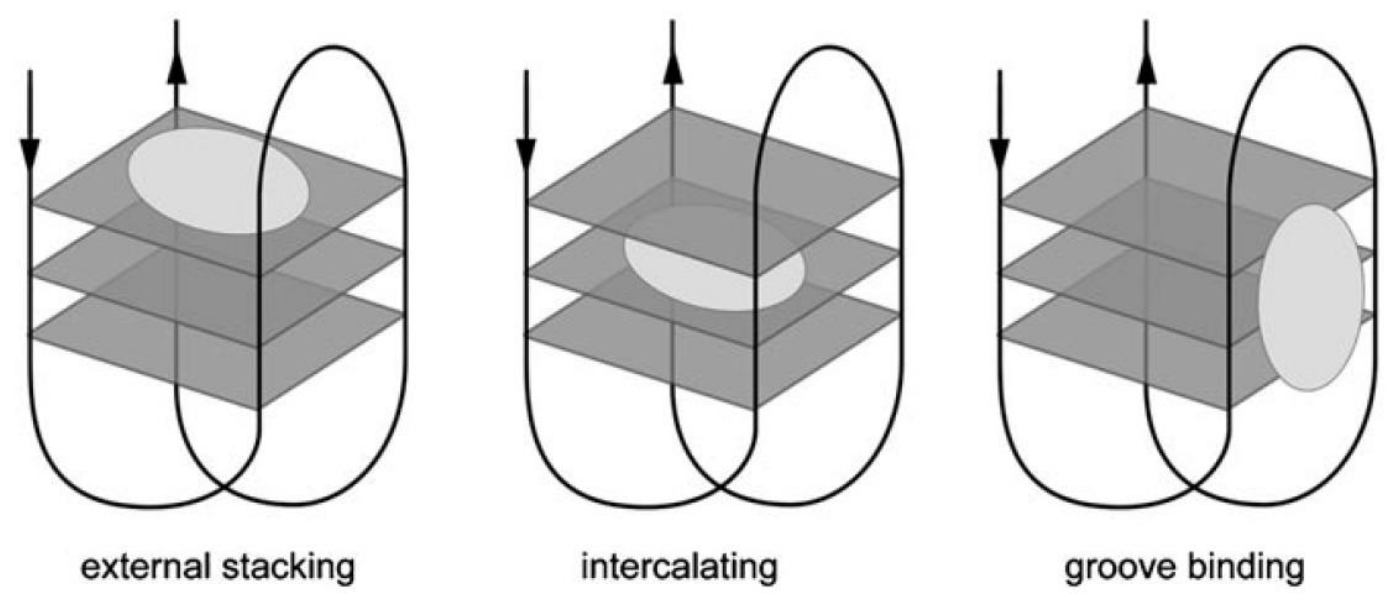

Figure 4-4 The different binding mode of metal complex to G-quadruplex. The graph was taken from ref 9

The earliest example of metal complex as G-quadruplex stabilizer was metalloporphyrin $^{10,11}$. Its comparable size with G-quartet and large delocalized $\pi$ electrons make it a proper candidate. It usually binds onto the external layer of G-quadruplex with the metal center locating above the G-quartet. Of its family, TMPyP4 (meso-methylpyridinium-substitued prophyrin) (figure 4-5) has been reported to possess a strong binding affinity towards G-quartet.

Apart from macrocyclic porphyrin ligands, other nonmacrocyclic polydentate ligands were also explored. Unlike the macrocyclic ligand which possesses planar structure intrinsically, such noncyclic ligand can become planar only with the appearance of metal center and is uaually non-planar otherwise. Besides, its smaller size makes it easier to intercalate into the G-quartet layers or bind onto the groove. 
Salphen $^{12}$, ter-pyridine ${ }^{13}$ and phenanthroline ${ }^{14}$ (figure 4-5) complexes are successful examples for this category.

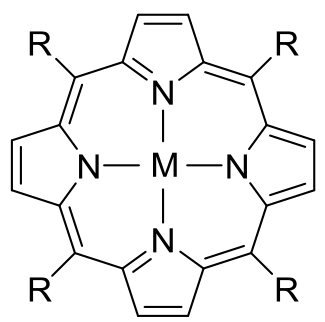

B<smiles>[M]OCc1ccccc1/C=N\c1ccccc1/N=C/c1ccccc1OC</smiles>

C<smiles></smiles>

D<smiles></smiles>

Figure 4-5 The different structure of metal complex. A: porphyrin; B: salphen; C: ter-pyridine; D: phenanthroline.

\subsubsection{Direct coordination of the metal to G-quadrulex}

Besides indirect interaction between metal-complex and G-quadruplex mentioned above, the metal center can also form coordination covalent bond with guanine. Actually many studies have shown that Pt(II) complex can easily coordinate to DNA nucleobase, especially N7 of guanine ${ }^{15-17}$. Under such circumstances, the chloride anion which initially coordinates to $\mathrm{Pt}(\mathrm{II})$ can be easily substituted. Unlike indirect metal complex which possess big delocalized structure, the direct coordination metal complexes don't require an aromatic structure in nature. According to the number of chloride coordinating to $\mathrm{Pt}(\mathrm{II})$, the complex can either target to one base site or crosslink two different bases.

An interesting example is to combine two types, either direct or indirect one, into one structure. As figure 4-6 shows, a clamp-like metal complex can bind onto both top and bottom of G-quadruplex at the same time ${ }^{18}$, with one end bearing a chloride coordinated Pt(II) and one end bearing a quinacridine aromatic ring. These two parts 
are linked by an alkyl chain which matches the height of G-quadruplex. Such a smart structure may provide double stabilization effect by different binding mechanisms.

However, it should be noted such direct coordination sometimes won't stabilize G-quadruplex, instead, it was found that cis- and trans-platin may induce unfolding of Tel-1 and Tel-2 quadruplex. While such an unfolding phenomenon was not found in in other types of G-quadruplex ${ }^{19}$, the reason may be other guanines other than G-quartet region in these structures are more accessible for Pt complex.
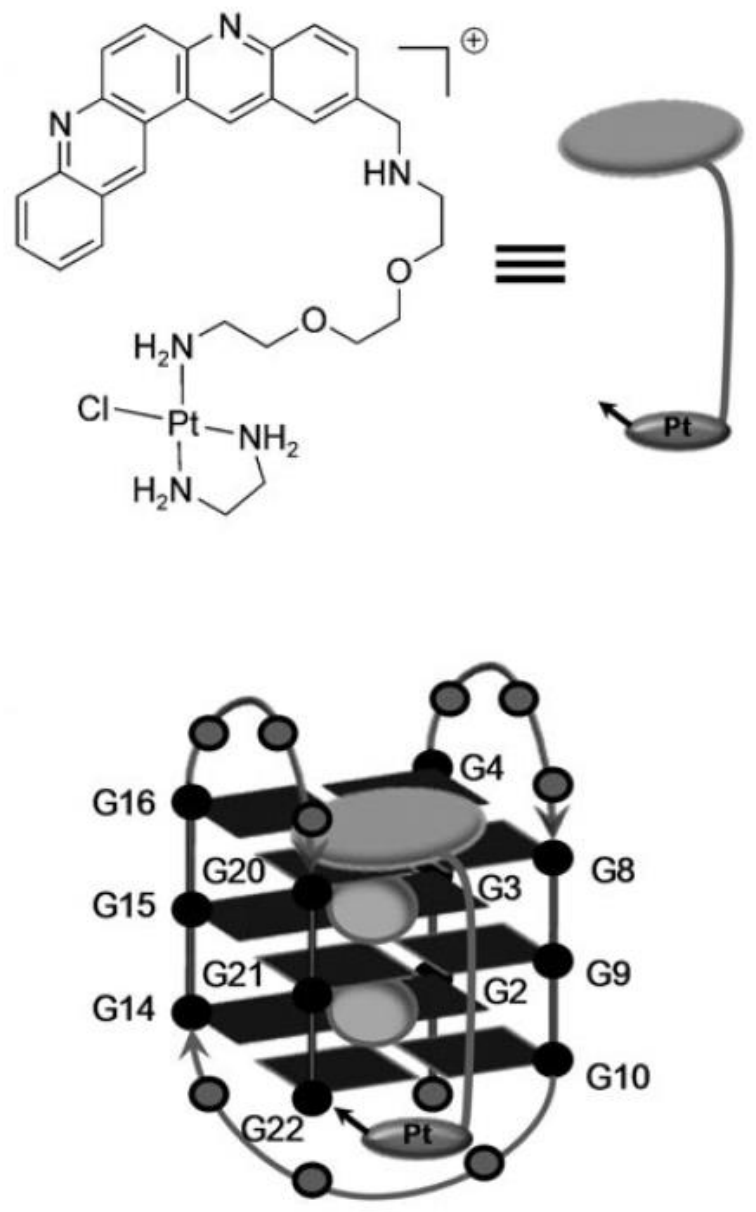

Figure 4-6 The bi-functional Pt(II) complex which can bind to both top and bottom G-quartet layers of G-quadruplex. The graph was taken from ref 20 
From the above introduction, it was demanding to explore the novel metal complex which possesses outstanding binding affinity as well as selectivity for different kinds of G-quadruplexes. In previous studies our group has synthesized varieties of metal complex and tested their stabilization effect towards G-quadruplex, in the following sections the related part will be introduced and the theoretical work to illustrate experimental data will be discussed in detail.

\subsubsection{The theoretical methods to explore G-quadruplex stabilizer}

Current theoretical methods are focusing on both prediction and confirmation. computer-aided drug design (CADD) was used extensively to help to filter out the promising candidate compound targeting various biomolecules, including G-quadruplex ${ }^{21}$. In the meanwhile the similar docking simulation accompanied by MD simulation was used as a theoretical explanation for experimental results ${ }^{22-24}$. In this chapter, we will mainly employ docking method as a tool to estimate the binding affinity of different metal complexes to G-quadruplex. MD simulation was used to construct the possible structure for dinucear complex crosslinked G-quadruplex.

Though much docking simulation has been done accompanied with experimental work, there seems no agreement on the performance of different softwares. GOLD is an effective and efficient software for this field, and it was proved that it can achieve rather reasonable predicted binding conformation for protein binder ${ }^{25}$. As a result, we will conduct docking simulation using this software in later sections. 


\subsection{Aza-bridged bisphenanthrolinyl Pt(II) complexes: efficient stabilization and topological selectivity on telomeric G-quadruplexes}

$\mathrm{Pt}(\mathrm{II})$ complex with phenathroline as ligand was proved to be an excellent G-quadruplex binder ${ }^{14}$. Our group tried to improve the stabilization of such complex by expand the delocalized area. Here, one more phenanthroline was introduced, and two phenanthrolines were linker by an aza bridge, as figure 4-7 shows. With the appearance of $\mathrm{Pt}(\mathrm{II})$, two phenanthrolines will form a rigid flat geometry and thus provide strong $\pi$ - $\pi$ stacking with G-quartet to enhance G-quadruplex stability.

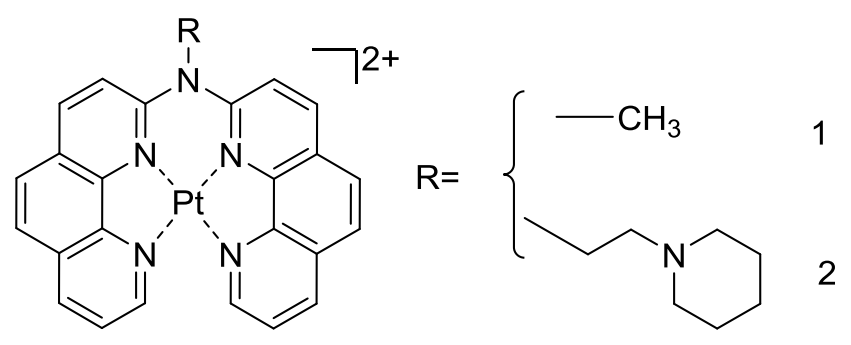

Figure 4-7 The structure of novel Pt(II) complex with different side chains.

\subsubsection{Experimental motivation}

Circular dichroism melting study showed the excellent selectivity of bisphenanthrolinyl Pt(II) complexes. Here we applied our complexes onto 4 different sequences: HT21 (human telomeric G-quadruplex), c-myc (DNA promoter G-quadruplex), TERRA (RNA G-quadruplex) and ds26 (duplex DNA). The results were shown in table 4-1. We noticed that melting temperature of HT21 can be increased by around $40{ }^{\circ} \mathrm{C}$ after adding either complex. In comparison, only 
moderate increment of melting temperature was seen for c-myc and TERRA sequence; furthermore, no significant change was found in normal duplex DNA.

In addition, we found such complexes showed preference to stabilize antiparallel G-quadruplex. As shown in figure 4-8, the CD spectrum pattern of hybrid form G-quadruplex changed into that of antiparallel form after adding the complex, with a new peak appeared at $260 \mathrm{~nm}$ and a slight shift down at $295 \mathrm{~nm}$. This result implied that upon binding, the antiparallel form may be more stable than initial hybrid form. On the contrary, no such induction effect was found for parallel c-myc sequence. To the best of our knowledge, no Pt complex has been reported to have the same induction effect towards human telomeric G-quadruplex. Such experimental results deserve further theoretical study.

Table 4-1 The increments in melting temperatures $\left(\Delta T_{\mathrm{m}}\right)$ of DNA by 1 and 2. $\Delta T_{\mathrm{m}}=$ $T_{\mathrm{m}}(\mathrm{Pt}-\mathrm{DNA})-T_{\mathrm{m}}$ (DNA), where $T_{\mathrm{m}}$ (Pt-DNA) and $T_{\mathrm{m}}$ (DNA) are CD melting temperatures of DNA structures in the presence and absence of platinum complexes, $\mathbf{1}$ or $\mathbf{2}$, respectively.

\begin{tabular}{ccc}
\hline & \multicolumn{2}{c}{$\Delta T_{\mathrm{m}}\left({ }^{\circ} \mathrm{C}\right)$} \\
\cline { 2 - 3 } DNA & 1 & 2 \\
\hline HT21 & $40(4)$ & $38(4)$ \\
$c-m y c$ & $16(3)$ & $16(4)$ \\
TERRA & $15(2)$ & $16(3)$ \\
ds26 & $4.5(0.8)$ & $2.0(0.6)$ \\
\hline
\end{tabular}



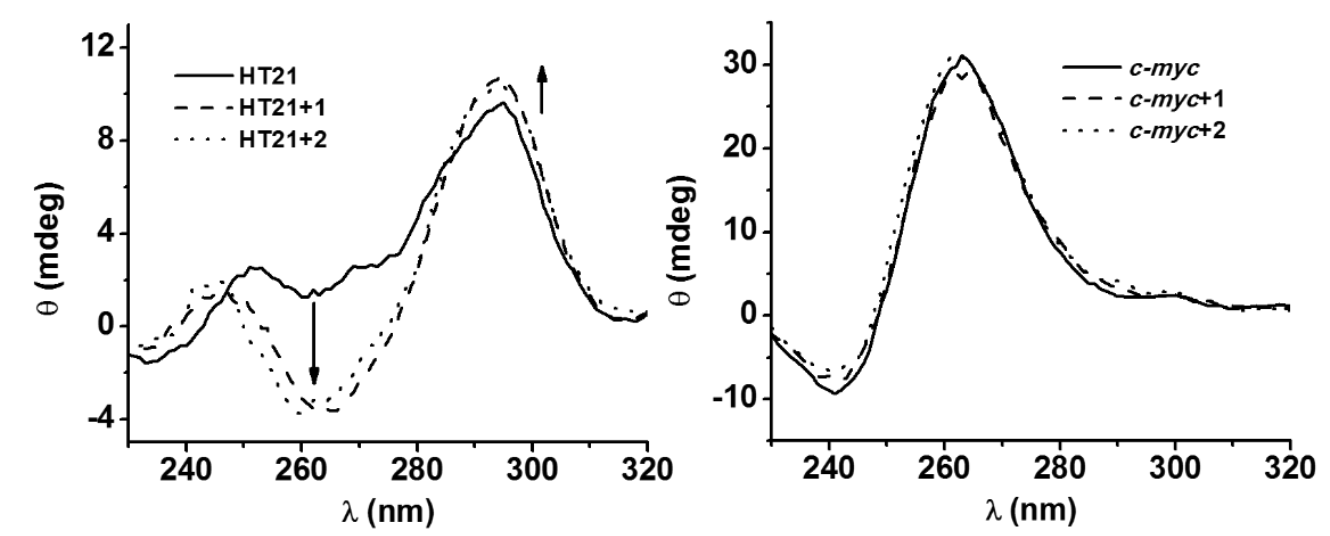

Figure 4-8 CD spectra of G-quadruplexes in the presence of 1 and 2. Left: HT21. Right: $\boldsymbol{c}-\boldsymbol{m y c}$.

\subsubsection{Theoretical methods}

\subsubsection{Structure optimization}

Since no experimental structure was available for complex 1 and 2, their structure was simulated in silico. The Gaussian view (Semichem Inc., Shawnee Mission, KS) ${ }^{26}$ was used to draw the initial structure, which was subject to structure optimization (B3LYP/6-31g* for $\mathrm{C}, \mathrm{H}, \mathrm{N}$; SDD for Pt) using Gaussian 09 (Gaussian, Inc., Wallingford, CT) ${ }^{27}$.

\subsubsection{Molecular docking}

The optimized complex structure underwent molecular docking using GOLD v5.4 (CCDC Software Limited) $^{28,29}$. We docked complex 1 and 2 into the antiparallel (PDB ID: 2MCC) and hybrid (PDB ID: 2MB3) G-quadruplex, respectively. Besides, these two complexes were also docked to parallel c-myc structure (PDB ID: 1XAV). The binding site was set to be the center of top layer G-quartet and the binding radius was set to be $15 \AA$. The fitness value was evaluated by CHEMPLP and for each 
docking simulation 10 repeats were conducted. The result with largest fitness value was selected and visualized by VMD (NIH Center for Macromolecular Modeling and

Bioinformatics, at the Beckman Institute, University of Illinois at Urbana-Champaign) $)^{30}$.

\subsubsection{Results and discussion}

\subsubsection{The selectivity of Pt complex for different G-quadruplexes}

The best docking results for complex 1 and 2 into different G-quadruplex were listed in table 4-2. From the table, both complexes showed rather poor affinity towards c-myc G-quadruplex, with the fitness value around 60. On the other hand, the fitness values for both folding structures of human telomeric sequence were more than 83, which suggested the obvious preference of the complex to human telomeric G-quadruplex. Moreover, such selectivity became even more obvious for complex 2, with fitness value difference more than 30 . The fitness value difference between HT and c-myc were consistent with CD melting data. The fitness value of complex 2 was larger than complex 1 when docked into the same G-quadruplex, which suggested the extra stabilization effect of complex 2. We distributed such extra stabilization effect to the interaction between alkyl side chain and loop of G-quadruplex. Whereas such difference was not found in experimental data, in which complex 1 and 2 showed almost the same $\Delta \mathrm{Tm}$.

Besides, the complex showed affinity to antiparallel type G-quadruplex than hybrid one. For both complexes, higher fitting scores were obtained for antiparallel 
G-quadruplex than those of hybrid topology. However the fitness value difference for complex 2 (106.1 in antiparallel type versus 94.1 in hybrid type) was 5 folds of that for complex 1 (89.6 in antiparallel type versus 83.4 in hybrid type).

Table 4-2 Best fitting values of molecular docking of $\mathbf{1}$ and $\mathbf{2}$ on various GQ topological structures

\begin{tabular}{cccc}
\hline $\begin{array}{c}\text { GQ } \\
\text { topology } \\
\text { (PDB code) }\end{array}$ & $\begin{array}{c}\text { antiparallel type } \\
(2 \mathrm{MCC})\end{array}$ & $\begin{array}{c}3+1 \text { hybrid } \\
(2 \mathrm{MB} 3)\end{array}$ & $\begin{array}{c}\text { parallel } c-m y c \\
(1 \mathrm{XAV})\end{array}$ \\
\hline $\mathbf{1}$ & 89.6 & 83.4 & 65.9 \\
$\mathbf{2}$ & 106.1 & 94.1 & 62.4 \\
\hline
\end{tabular}

\subsubsection{The stabilization effect of alkyl side chain}

From table 4-2, we found complex 2 may enhance the fitness value difference between antiparallel and hybrid G-quadruplexes. Such discrepancy was due to the interaction between the cycloalkyl group and the loop bases of G-quadruplex. From the visualization graph, this point can be illustrated more clearly. From the figure 4-9 and 4-10, a quick glance showed that both complex 1 and 2 can stack well onto the top of 2 types of G-quadruplexes. The area of bisphenanthroline was at the same scale as that of G-quartet, which made the stacking easy to take place. Interestingly, we found that when complex 2 docked into antiparallel-type G-quadruplex (figure 4-10a), its alkyl side chain will stretch out and interact with the adenine on the loop. The hydrogen atoms on the cyclohexanyl group were in vicinity close enough with nitrogen atoms within adenine to form hydrogen bond (figure 4-10b). While such interaction between side chain and loop base was not found in the hybrid-type 
G-quadruplex, in which the alkyl side chainonly point out into the empty space between loop backbones (figure 4-10c). Such difference was based on the topology of two G-quadruplexes and was reflected on the difference between fitness values of two complexes.

In conclusion, by docking simulation we illustrate the selectivity of novel $\mathrm{Pt}(\mathrm{II})$ complex to human telomeric G-quadruplex. The fitness value well matched the melting temperature trend as well as the CD spectrum transition. By docking simulation, we demonstrate the effect of alkyl side chain to further stabilize G-quadruplex.
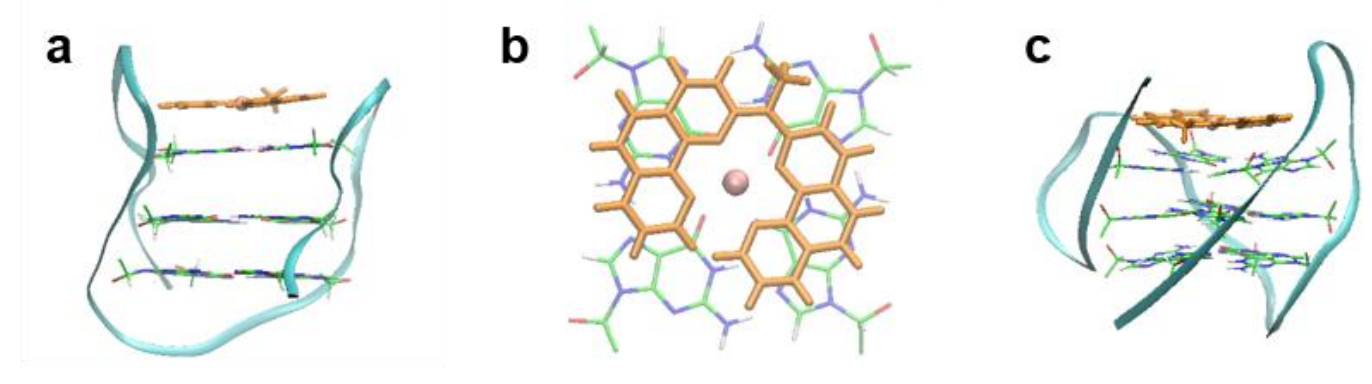

Figure 4-9 Molecular docking of $\mathbf{1}$ on antiparallel ( $a$ : side view; $b$ : top view,) and $3+1$ hybrid GQ ( $c$ : side view). Backbone and G-quartets in both quadruplexes were represented as cyan ribbon and in stick mode, respectively. Ligands and Pt cation were shown in orange stick and pink ball, respectively. 

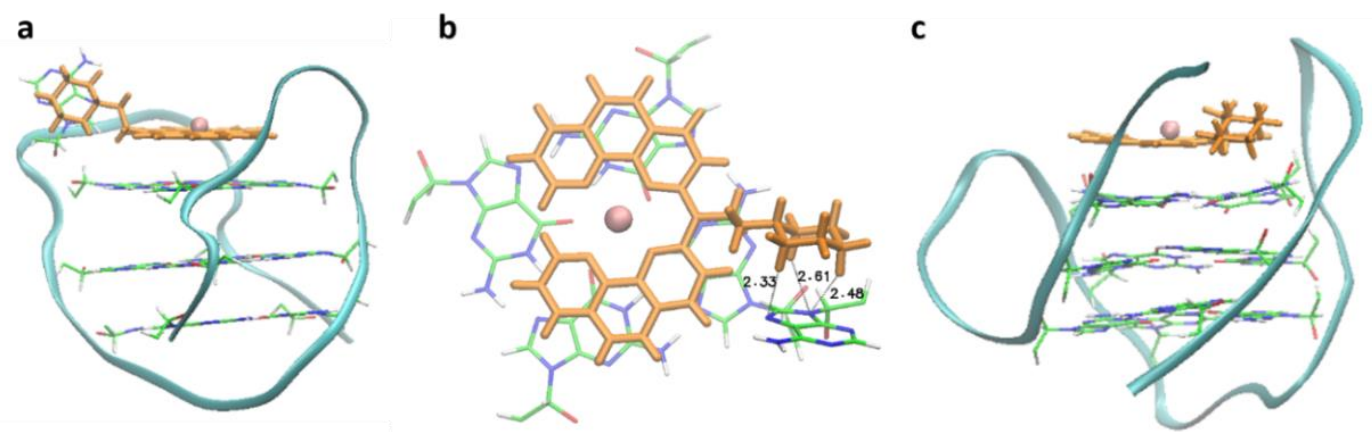

Figure 4-10 Molecular docking of 2 on antiparallel ( $a$ : side view; $b$ : top view,) and 3+1 hybrid GQ ( $c$ : side view). Backbone and G-quartets in both quadruplexes were represented as cyan ribbon and in stick mode, respectively. Ligands and Pt cation were shown in orange stick and pink ball, respectively.

\subsection{Octahedral ruthenium complexes selectively stabilize G-quadruplexes}

Ruthenium(II) is also a widely used metal center to form complex and there are many reports about the stabilization effect of such complexes ${ }^{31-33}$. Unlike $\mathrm{Pt}(\mathrm{II})$, the octahedral coordination geometry of $\mathrm{Ru}(\mathrm{II})$ provides extra possibility to adjust the ligand outside the planar part. However, previous $\mathrm{Ru}(\mathrm{II})$ complexes are all possess $\mathrm{C} 3$ symmetry with tris-bidentate ligands, such complexes usually show more loop/groove affinity.

Here, our group designed and synthesized a novel $\mathrm{Ru}(\mathrm{II})$ complex with $\mathrm{C} 4$ symmetry, as figure 4-11 shows. The bisphenanthroline was selected to provide the planar ligands, while the top and bottom ones were treated differently either with two ammonia or one ammonia and one DMSO. The large delocalized area from bisphenanthroline can provide the complex the affinity to stack onto G-quartet instead 
loop or groove region, at the same time, such large aromatic area may avoid the complex to intercalate into duplex and bestow it the selectivity to G-quadruplex.

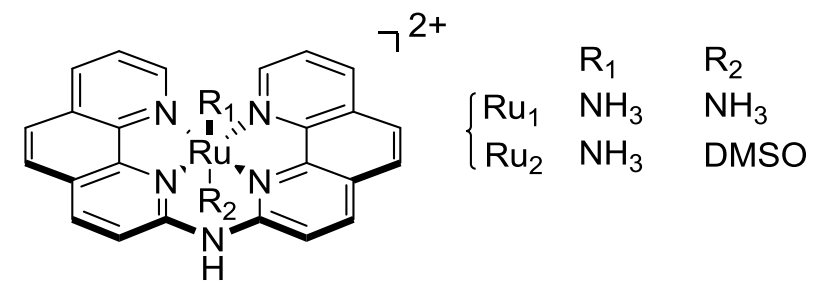

Figure 4-11 Structure of $\mathrm{Ru}(\mathrm{II})$ complex with different $\mathrm{z}$-axis ligands.

\subsubsection{Experimental motivation}

$\mathrm{CD}$ melting was conducted to distinguish the binding affinity of Ru1 to G-quadruplex and duplex. Here the target DNA were HT21 (human telomeric G-quadruplex), c-myc (promoter G-quadruplex) and DS26 (duplex DNA). The results were shown in table 4-3. From the table it's clear Ru1 has much stabilization effect to HT21, whose $\Delta \mathrm{Tm}$ can reach up to $26^{\circ} \mathrm{C}$. However, for c-myc only moderate shift up $\left(\sim 10{ }^{\circ} \mathrm{C}\right)$ of $\Delta \mathrm{Tm}$ was observed. On the contrary, Ru2 which possessed different ligands on z-axis showed less stabilization effect to both HT21 and c-myc G-quadruplex. Moreover, the selectivity of Ru2 to HT21 was also lost, with almost the same $\Delta \mathrm{Tm}$ for both G-quadruplex. For duplex only negligible increase of melting temperature was found.

Table 4-3 The increments in melting temperatures $(\Delta \mathrm{Tm})$ of DNA by Ru1 and Ru2. $\Delta \mathrm{Tm}=\mathrm{Tm}(\mathrm{Ru}-\mathrm{DNA})-\mathrm{Tm}(\mathrm{DNA})$, where $\mathrm{Tm}(\mathrm{Ru}-\mathrm{DNA})$ and $\mathrm{Tm}(\mathrm{DNA})$ are CD melting temperatures of DNA structures in the presence and absence of platinum complexes, Ru1 or Ru2, respectively. 


\begin{tabular}{ccc}
\hline & \multicolumn{2}{c}{$\Delta T_{\mathrm{m}}\left({ }^{\circ} \mathrm{C}\right)$} \\
\cline { 2 - 3 } DNA & $\mathrm{Ru} 1$ & $\mathrm{Ru} 2$ \\
\hline HT21 & $26(2)$ & $9.6(0.8)$ \\
$c$ - $m y c$ & $10(2)$ & $7.2(0.5)$ \\
ds26 & $1.9(0.4)$ & $1.2(0.3)$ \\
\hline
\end{tabular}

Furthermore, the same induction effect as Pt(II) complex mentioned above was observed. As figure 4-12 shows, when titrating Ru1 into the HT21, two characteristic MLCT (Metal-to-ligand charge transfer) bands suffered significant alterations: 1) the intensity of MLCT band from $344 \mathrm{~nm}$ to $363 \mathrm{~nm}$ decreased greatly, suggesting the strong $\pi$-stacking between bisphenanthroline and G-quartet; 2) at the same time the bind at $513 \mathrm{~nm}$ underwent large redshift and hypochromicity, which indicated the dampened interaction between $\mathrm{NH}_{3}$ and $\mathrm{Ru}(\mathrm{II})$. However for duplex DNA, we didn't observe such transition. Such distinct phenomena revealed the selectivity of Ru1 to HT21 G-quadruplex.
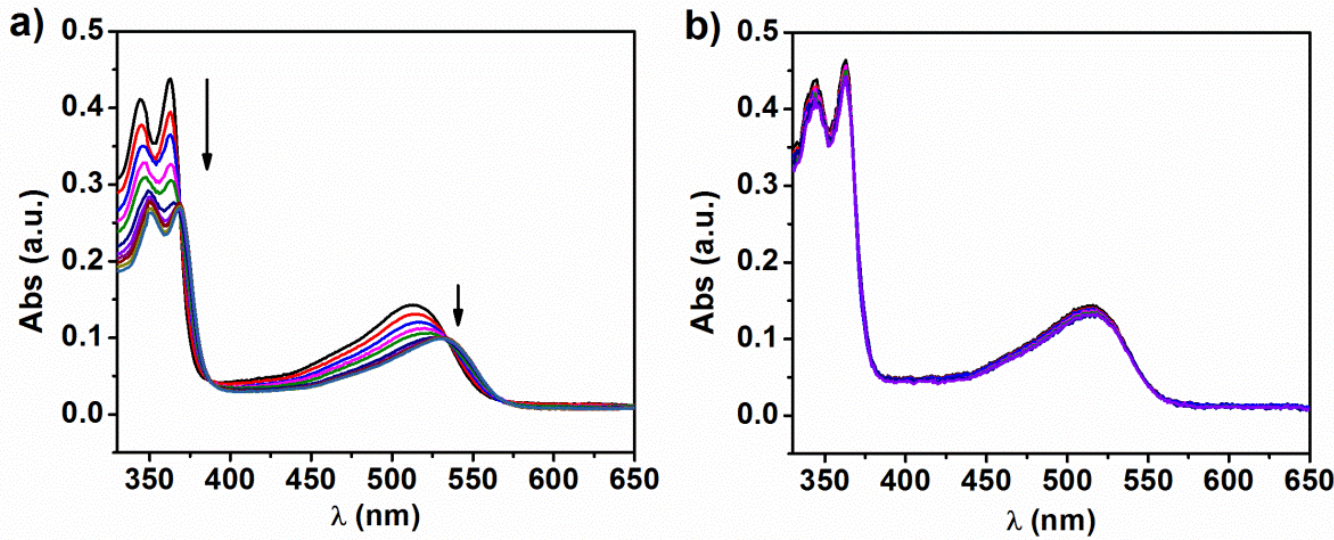

Figure 4-12 UV-vis titration spectra of Ru1 (25 mM) with increasing concentrations of GQ HT21 (black to dark cyan: 0 to $8 \mathrm{mM}$ ) (a) and DS26 (black to violet: 0 to 12 
$\mathrm{mM})(\mathrm{b})$.

\subsubsection{Theoretical investigation}

The structure of Ru1 and Ru2 were obtained readily from X-ray scattering experiment. For each complex, we docked it into the antiparallel (PDB ID: 2MCC) and hybrid (PDB ID: 2MB3) human telomeric G-quadruplex, or parallel c-myc G-quadrplex (PDB ID: 1XAV), respectively using GOLD v5.4 software ${ }^{28,29}$. The binding site was set to be the center of top G-quartet and the pocket radius was set to be $15 \AA$. The scoring function was CHEMPLP and for each docking simulation 10 runs were conducted. The result with highest fitness value was selected and visualized using $\mathrm{VMD}^{30}$.

The docking results were shown in table 4-4. The fitness value of Ru1 docked into c-myc G-quadruplex was only 60.57, while that of Ru1 docked into human telomeric G-quadruplex (both antiparallel and hybrid type) were more than 88 , which illustrate the selectivity of Ru1 to HT G-quadruplex. Moreover, compared with Ru1, Ru2 showed less stabilization effect to HT G-quadruplex. The fitness value of Ru2 was slightly less than that of Ru1 (less than 2.0) when docked into antiparallel type G-quadruplex, while such difference was much more pronounced ( 6.0) when we docked them into hybrid type. We distribute less fitness of Ru2 to the repulsion between bulky DMSO ligand and loop region of G-quadruplex. The trend of fitness value was consistent with that of $\mathrm{CD}$ melting data. 
The transition from hybrid to antiparallel type G-quadruplex can be illustrated by the higher fitness value of antiparallel over hybrid type G-quadruplex: both Ru1 and Ru2 showed preference to antiparallel type, which indicate the antiparallel type was more thermodynamically stable.

The visualization of docking results was shown in figure 4-13 and 4-14. The bisphenanthroline plane stacked well with G-quartet with the similar area scale. Interestingly, for both $\mathrm{Ru} 1$ and $\mathrm{Ru} 2$, the $\mathrm{z}$-axis ammonia always pointed down with its hydrogen atom close enough to G-quartet to form hydrogen bond. Here the effect of $\mathrm{NH}_{3}$ was very similar to that of $\mathrm{K}^{+}$in ion channel. The formation of hydrogen bond enhanced the interaction of $\mathrm{NH}_{3}$ and G-quartet and weakened the chelation of $\mathrm{NH}_{3}$ to $\mathrm{Ru}(\mathrm{II})$. This theoretical phenomenon was consistent with the reduction of MLCT band at 344-363 nm. For Ru2 we didn't find DMSO-down conformation, since the bulky structure of DMSO may raise the repulsion between the ligand and G-quadruplex. The interaction between $\mathrm{NH}_{3}$ and G-quartet may provide extra stabilization apart from the $\pi$-stacking between bisphenanthroline and G-quartet.

Table 4-4 Best fitting values of molecular docking of Ru1 and Ru2 on various GQ topological structures.

\begin{tabular}{cccc}
\hline $\begin{array}{c}\text { GQ } \\
\text { topology } \\
\text { (PDB code) }\end{array}$ & $\begin{array}{c}\text { antiparallel type } \\
(2 \mathrm{MCC})\end{array}$ & $\begin{array}{c}3+1 \text { hybrid } \\
(2 \mathrm{MB} 3)\end{array}$ & $\begin{array}{c}\text { parallel } c-m y c \\
(1 \mathrm{XAV})\end{array}$ \\
\hline Ru1 & 94.21 & 88.06 & 60.57 \\
Ru2 & 92.91 & 82.59 & 55.22 \\
\hline
\end{tabular}



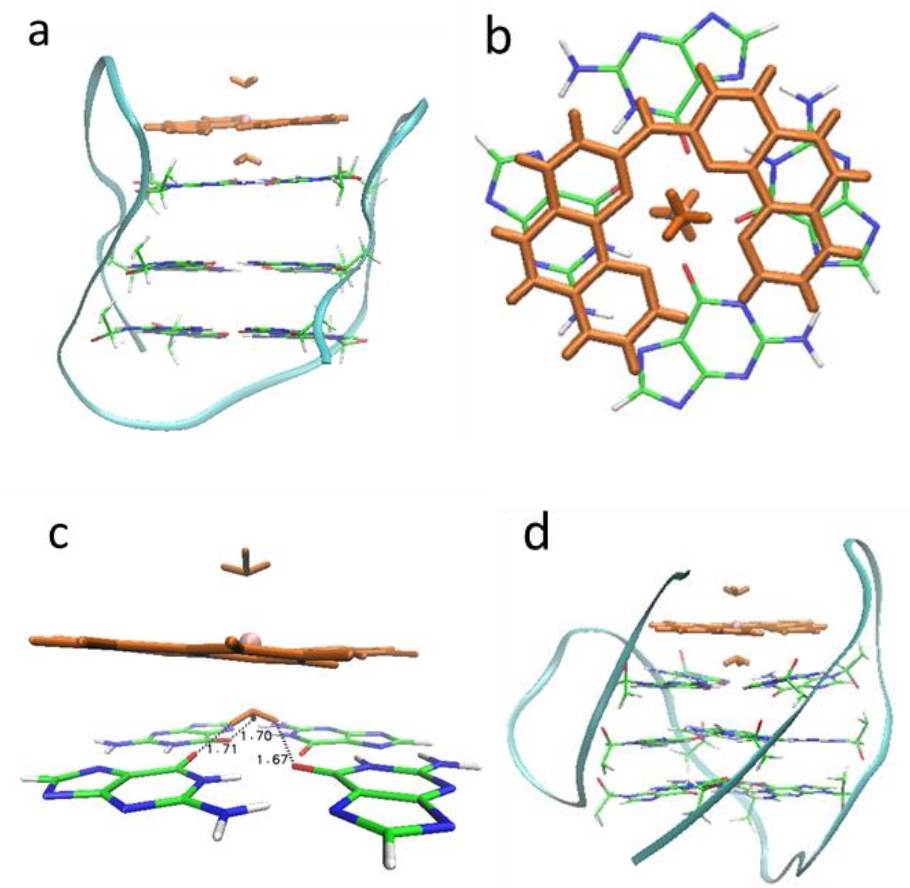

Figure 4-13 Molecular docking of Ru1 on antiparallel GQ (a: side view; b: top view of complex and top G-quartet; c: side view of complex and top G-quartet) and on hybrid GQ (d: side view). Backbone is represented as a cyan ribbon; Ligands and Ru cation were shown in orange stick and pink ball, respectively.
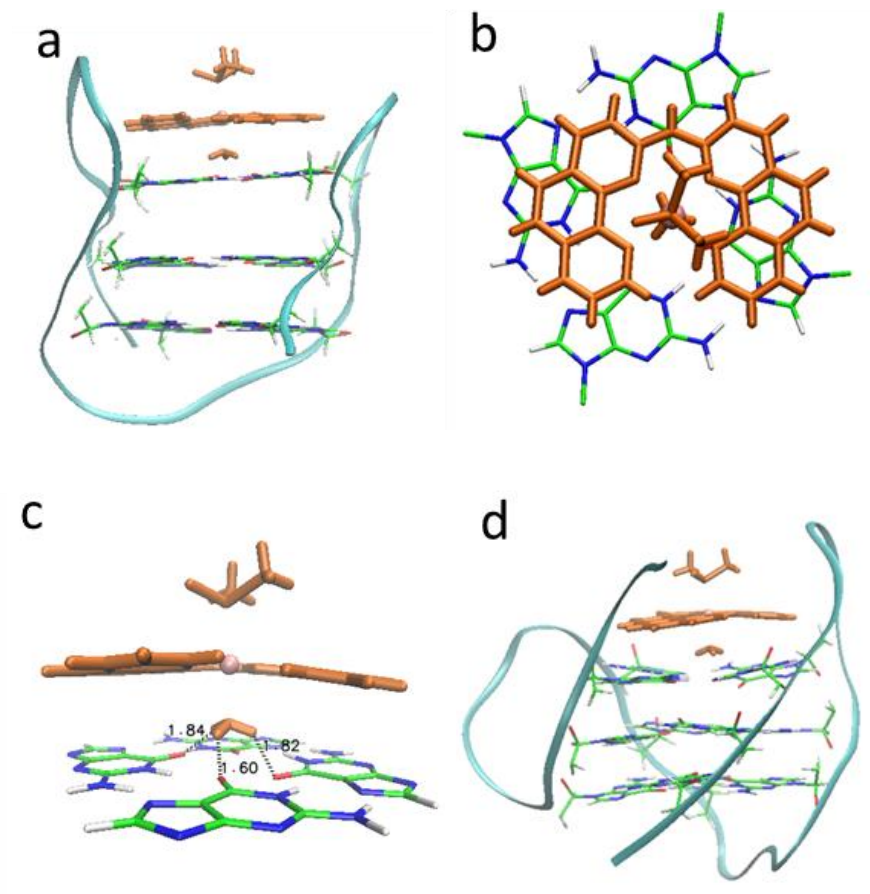
Figure 4-14 Molecular docking of Ru2 on antiparallel GQ (a: side view; b: top view of complex and top G-quartet; c: side view of complex and top G-quartet) and on hybrid GQ (d: side view). Backbone is represented as a cyan ribbon; Ligands and $\mathrm{Ru}$ cation were shown in orange stick and pink ball, respectively.

In conclusion, the binding affinity of novel $\mathrm{Ru}(\mathrm{II})$ complex to human telomeric G-quadruplex was illustrated both experimentally and theoretically. The fitness value of docking simulation explained the CD melting pattern and CD curve transition from hybrid to antiparallel. The visualization showed the stabilization of such $\mathrm{Ru}(\mathrm{II})$ complex may come from the hydrogen bond between ammonia ligand and G-quartet.

\subsection{Dinuclear platinum compounds specially cross-link to parallel human c-myc G-quadruplex}

Planar Pt(II) complex has already seen many successful examples, such as Pt-dppz ${ }^{34}$, Pt-terpyridine ${ }^{35}$ and Pt-phenanthroline ${ }^{36}$. Meanwhile, the complex with double binding sites was also mentioned above. Recently, dinuclear and multinuclear $\mathrm{Pt}(\mathrm{II})$ complexes were reported to be an effective telomerase inhibitors and GQ binder $^{23}$. Inspired by such work, we designed and synthesized a novel dinuclear Pt(II) complex using phenanthroline as ligands and chloride as leaving group, as shown in figure 4-15. The two planar Pt(II) ligands were connected by flexible alkyl chain, and for each Pt(II) complex, the chloride can be substituted by the N7 atom of guanine to form crosslink product. 


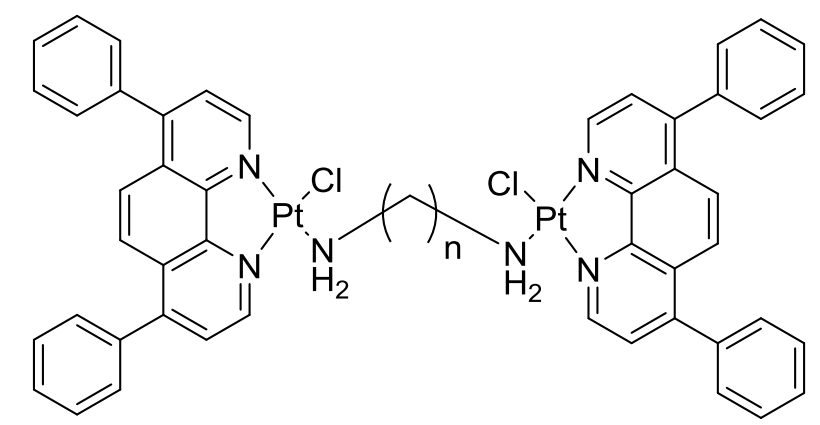

Figure 4-15 Scheme for structures of dinuclear platinum compounds, named Pt1 to Pt4 with $\mathrm{n}$ from 7 to 10

\subsubsection{Experimental motivation}

The CD melting study was first conducted to investigate the stabilization effect of the complex. The results were shown in table 4-5. Interestingly, the complex didn't show significant binding affinity towards c-myc G-quadruplex. And $\Delta \mathrm{Tm}$ changes according to the length of alkyl chain, among which the Pt3 with 9 carbon alkyl chain showed the most stable binding affinity. The result indicated the importance of linker which controlled the distance between two Pt(II) complexes. If the length was too short or too long, the possibility of two terminal Pt(II) complexes to approach top and bottom G-quartet will be reduced.

Table 4-5 The increments in melting temperatures $\left(\Delta T_{\mathrm{m}}\right)$ of c-myc by Pt complexes. $\Delta T_{\mathrm{m}}=T_{\mathrm{m}}(\mathrm{Pt}-\mathrm{DNA})-T_{\mathrm{m}}(\mathrm{DNA})$, where $T_{\mathrm{m}}(\mathrm{Ru}-\mathrm{DNA})$ and $T_{\mathrm{m}}(\mathrm{DNA})$ are CD melting temperatures of DNA structures in the presence and absence of platinum complexes, respectively.

$$
\Delta T_{\mathrm{m}}\left({ }^{\circ} \mathrm{C}\right)
$$


Pt2

$\mathrm{Pt} 3$

$\mathrm{Pt} 4$

The coordinated site of c-myc was then explored further. The product after substitution was subjected to HPLC (High Performance Liquid Chromatography) for separation and two components were found besides c-myc G-quadruplex, as indicated as $\mathrm{P} 1$ and $\mathrm{P} 2$ in figure 4-16. All 3 peaks were characterized by MALDI-TOF (Matrix Assisted Laser Desorption/Ionization-Time of Flight) MS, which gave the same mass for $\mathrm{P} 1$ and $\mathrm{P} 2$. This result indicated both $\mathrm{P} 1$ and $\mathrm{P} 2$ were coordinated product with two crosslinking via two Pt core after substitution, while the conformation may be slightly different, which explained the small discrepancy of retention time. 

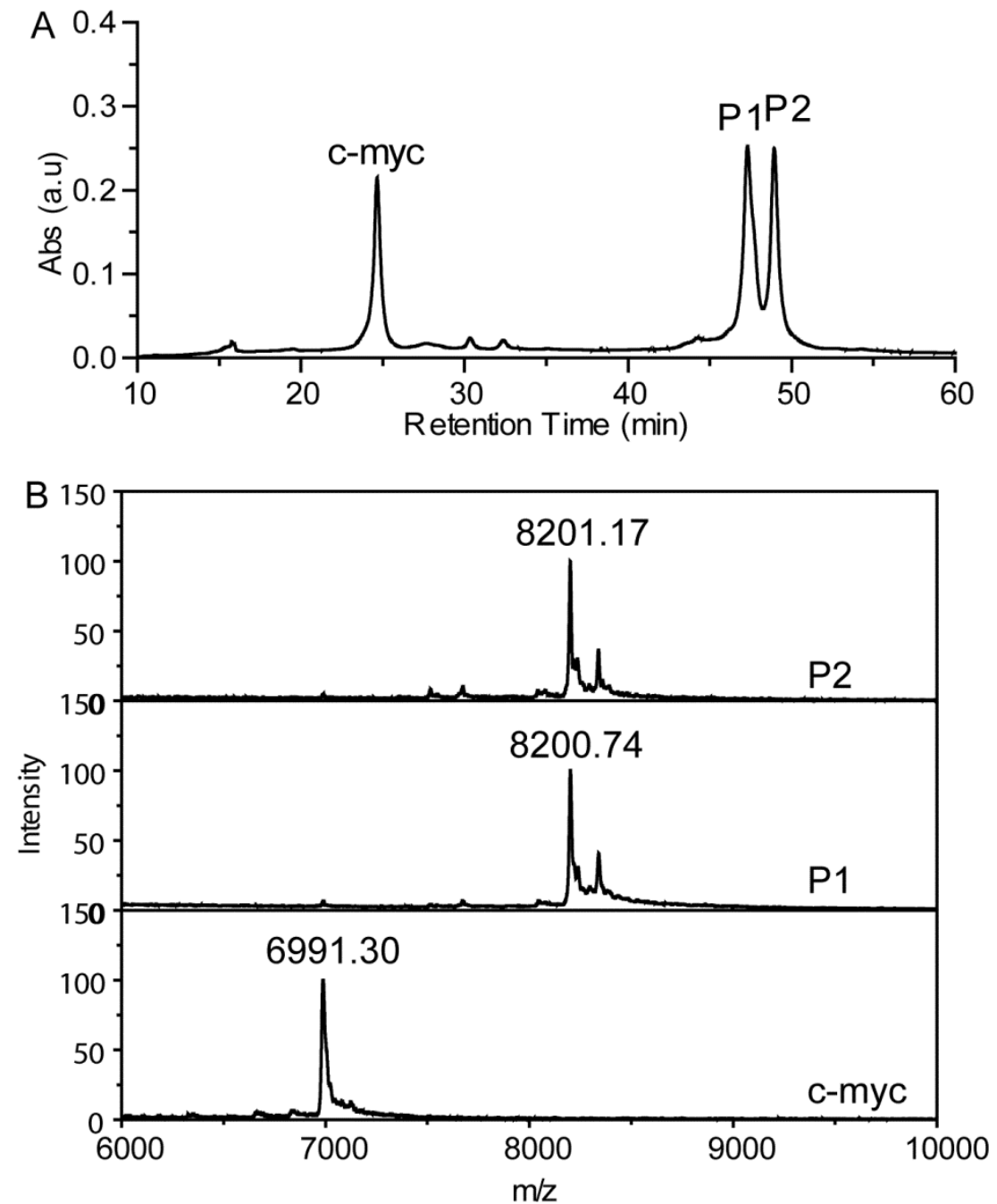

Figure 4-16 A: HPLC chromagraph for c-myc reacted with Pt3; B: MALDI-TOF MS verification of peaks from HPLC.

The separated P1, P2 and c-myc then underwent enzyme digestion followed by MALDI-TOF MS. The snake venom phosphodiesterase (SVP) and bovine spleen phosphodiesterase (BSP) were applied to digest the DNA/Pt(II) complex from $5^{\prime}$ and 3', respectively. The results were shown in figure 4-17. As shown in Figure A, both SVP and BSP can digest up to 7 bases of c-myc from both ends. However the existence of $\mathrm{Pt}(\mathrm{II})$ inhibited the digestion activity of both enzymes. For both products, BSP can only digest one base, which suggested the second guanine coordinated to 
Pt(II). While SVP showed different digestion product with regard to P1 and P2: for P1 we can only find one obvious peak which was confirmed to be the product after digestion of T20; on the other hand, 3 peaks were observed for digestion product of P2; besides T20, the peaks for A21 and A22 also appeared. Such disagreement between two products indicated the accessibility from 3' for P1 and P2 was different. The conformation of P1 may be more difficult for enzyme to approach terminal A21 and A22. While the assumption still required confirmation. The experimental methods were hard to detect the conformation of such coordinate product; instead, the structures in silico are constructed to explain above-mentioned phenomenon. 
$5^{\prime}-T_{1} G_{2} A_{3} G_{4} G_{5} G_{6} T_{7} G_{8} G_{9} G_{10} T_{11} A_{12} G_{13} G_{14} G_{15} T_{16} G_{17} G_{18} G_{19} T_{20} A_{21} A_{22}-3^{\prime}$
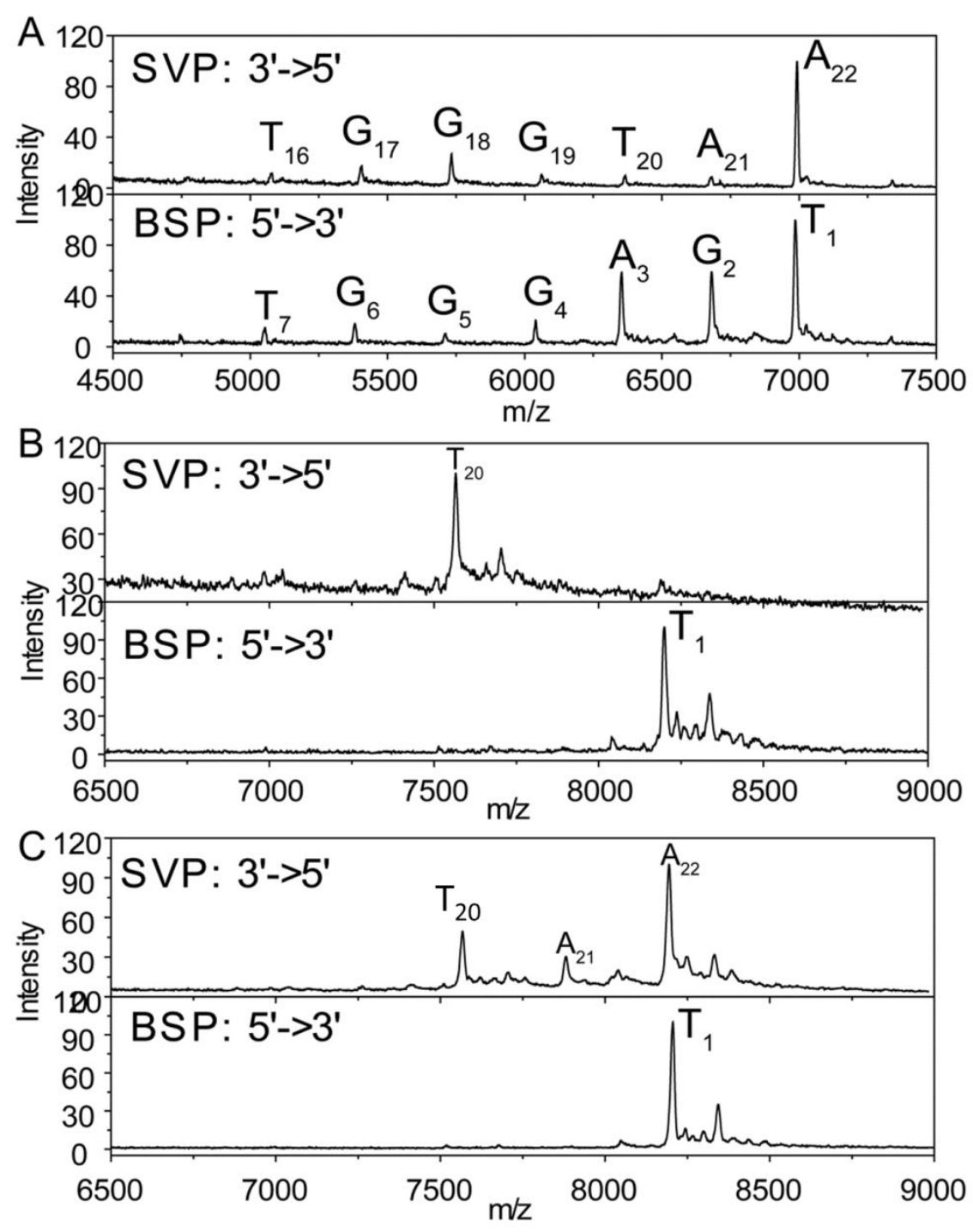

Figure 4-17 MALDI-TOF mass spectra of of c-myc (A) and Pt-adducts (B: P1; C: P2) after enzyme digestion. The letters indicate the new terminal of the DNA after digestion.

\subsubsection{Theoretical methods}

\subsubsection{Molecular docking}

The structure of Pt3 was drawn in Gaussian view ${ }^{26}$ and then optimized (B3LYP/6-31g* for C, H, N,Cl; SDD for Pt) in Gaussian 09. GOLD v5.4 $4^{28,29}$ was 
used to dock binuclear $\mathrm{Pt}(\mathrm{II})$ complex into the proximal position of c-myc G-quadruplex (PDB ID: 2L7V). To make the subsequent substitution easy to occur, one constraint of 5.0/ $\AA^{2}$ was applied between one Pt center and N7 of G2 and another constraint with the same value was applied between the other Pt and N7 of G19. Here the docking center was set to be the center of the whole c-myc G-quadruplex and docking radius was set to be $30 \AA$ to allow the software to explore all the possible binding sites of the acceptor. The scoring function was CHEMPLP and 30 runs were conducted. From the results two possible conformations were found and visualized using VMD, which were shown in figure 4-18. For both conformations, one end of $\mathrm{Pt}(\mathrm{II})$ complex fitted well into the bottom pocket with the bisphenanthroline plane stacking onto the bottom G-quartet, while the alkyl linkage either stretched across the loop of G-quadruplex (Conf 1) or resided along the groove (Conf 2) to lead the other Pt(II) complex to 3'-end of the G-quadruplex.
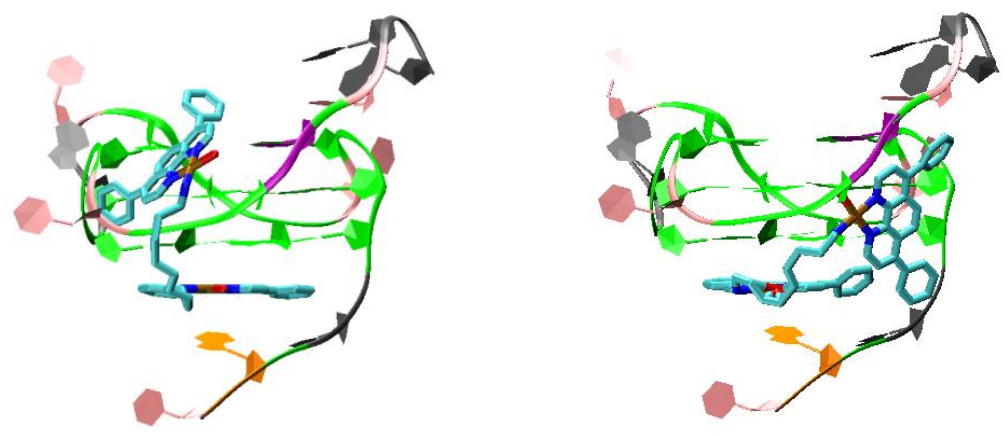

Figure 4-18 The molecular docking with restraint. Left: conf1; right: conf2. The G-quadruplex was represented using new ribbon method (guanine: green; adenine: gray; thymine; pink; G2: orange; G19: pruple). 


\subsubsection{Setup of Pt-guanosine force field}

From the docking structure, we noticed the bottom part of $\mathrm{Pt}(\mathrm{II})$ was ready to undergo substitution. To simulate the product after substitution, we need to build the force field of mono-substitution product first. The substituted structure was shown in figure 4-19. The force field for the DNA apart from substituted guanine can be obtained readily from AMBER99bsc0. With the help of MCPB.py ${ }^{37}$ in Ambertools16, the required force constant and atomic charge for substituted part can be calculated. The basic procedure was listed as followed.

To build up a complete force field for a new molecule, we needed to identify the parameters for both bonded terms and non-bonded terms. The non Pt-containing bonded terms can be easily achieved from existing force filed: for guanosine part (red color) we use Amber99bsc0 force field and for the rest ligands (black color) we use GAFF force field ${ }^{38}$. For the Pt-containing bonded terms, the equilibrium distance, angle and dihedral were first computed as the corresponding value of optimized structure, which was obtained using Gaussian 09 software. The force constant was achieved from the Hessian matrices ${ }^{39}$. With regard to non-bonded terms, the atomic charge was first evaluated using RESP method $^{40}$, the electrostatic potential information was first computed by MK method ${ }^{41}$ in Gaussian 09 and then the atomic charge was fitted using RESP program in Ambertools. The VDW parameters can be obtained readily from previous reference ${ }^{42}$. After that, some of the Pt containing terms were modified according to the reference ${ }^{43}$. The modified parameters were listed in table 4-6 to 4-10. 


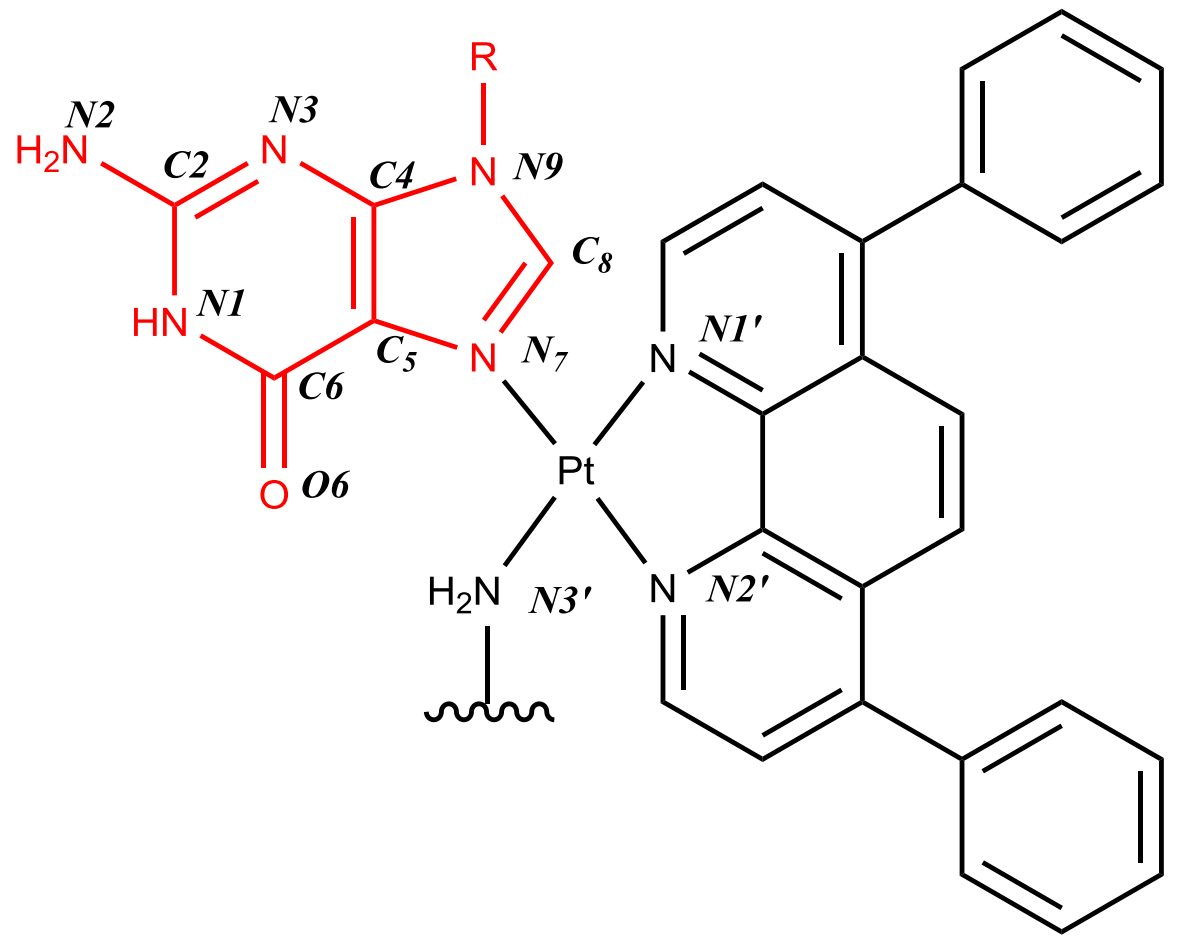

Figure 4-19 The structure of Pt-guanosine complex, the atom types of coordinate atoms and guanine atoms are also labeled.

Table 4-6 The modified bond terms for guanine-Pt complex

\begin{tabular}{lll}
\hline Bond & $\mathbf{R}_{\mathbf{0}}$ & $\mathbf{K}_{\mathbf{r}}\left(\mathbf{k J} /\left(\mathbf{n m}^{2} * \mathbf{m o l}\right)\right)$ \\
\hline $\mathbf{N 7 - P t}$ & 2.01 & $1.5313 \times 10^{5}$ \\
\hline
\end{tabular}

Table 4-7 The modified angle terms for guanine-Pt complex

\begin{tabular}{lll}
\hline Angle & $\boldsymbol{\theta}_{\mathbf{0}}$ & $\mathbf{K}_{\boldsymbol{\theta}}(\mathbf{k J} /(\mathbf{d e g r e e} * \mathbf{m o l}))$ \\
\hline N1'-Pt-N2' & 90 & $1.6688 \times 10^{3}$ \\
N1'-Pt-N3' & 180 & $1.6044 \times 10^{3}$ \\
N2'-Pt-N3' & 90 & $1.5382 \times 10^{3}$ \\
Pt-N7-C8 & 127.95 & $1.0385 \times 10^{3}$ \\
Pt-N7-C5 & 127.95 & $1.3339 \times 10^{3}$ \\
N1'-Pt-N7 & 90 & $1.3551 \times 10^{3}$ \\
N2'-Pt-N7 & 180 & $1.4041 \times 10^{3}$ \\
N3'-Pt-N7 & 90 & $1.4495 \times 10^{3}$ \\
\hline
\end{tabular}

Table 4-8 The modified dihedral terms for guanine-Pt complex

\begin{tabular}{llll}
\hline Torsional angle & $\boldsymbol{\varphi}_{\mathbf{0}}$ & $\mathbf{V n}(\mathbf{k J} / \mathbf{m o l})$ & $\mathbf{n}$ \\
\hline C5-C7-Pt-N1 & 90 & 2.09 & 2 \\
\hline
\end{tabular}




\begin{tabular}{llll}
\hline C8-C7-Pt-N1' & 90 & 2.09 & 2 \\
C5-C7-Pt-N2, & 90 & 2.09 & 2 \\
C8-C7-Pt-N2, & 90 & 2.09 & 2 \\
C5-C7-Pt-N3, & 90 & 2.09 & 2 \\
C8-C7-Pt-N3, & 90 & 2.09 & 2 \\
\hline
\end{tabular}

Table 4-9 The modified improper torsional terms for guanine-Pt complex

\begin{tabular}{lll}
\hline Improper angle & $\chi_{0}$ & $\mathbf{K}_{\chi}(\mathbf{k J} / \mathbf{m o l})$ \\
\hline C8-C7-C5-Pt & 180 & 41.8 \\
\hline
\end{tabular}

Table 4-10 The modified atomic charge for guanine-Pt complex

\begin{tabular}{ll}
\hline Atom & Atomic charge \\
\hline N9 & -0.019 \\
C8 & 0.3057 \\
H8 & 0.069 \\
N7 & -0.3433 \\
C5 & 0.009 \\
C6 & 0.6837 \\
O6 & -0.5699 \\
N1 & -0.5053 \\
H1 & 0.352 \\
C2 & 0.7432 \\
N2 & -0.923 \\
H21 and H22 & 0.4235 \\
N3 & -0.6636 \\
C4 & 0.3847 \\
N1' & -0.11232 \\
N2' & -0.13805 \\
N3' & -0.41289 \\
\hline
\end{tabular}

\subsubsection{Constructing mono-substituted product}

The chloride coordinate to bottom Pt was deleted manually and the new bond was added between Pt and N7 of G2. Such structure was first optimized using Discovery Studio $2016^{44}$ followed by MD simulation using GROMACS v4.6 package. The substituted structure was first subject to a PBC cubic box with the distance between solute and box side to be $1 \mathrm{~nm}$, the box was then filled with water and neutralized 
with $\mathrm{Na}^{+}$, and minimization, NVT $(298 \mathrm{~K}, 100 \mathrm{ps})$ and NPT (1 atm, $\left.100 \mathrm{ps}\right)$ equilibriums, were conducted sequentially. Thereafter the system was subject to a 10 ns product MD with a distance restraint of $1.0 \times 10^{3} \mathrm{~kJ} /\left(\mathrm{mol} \mathrm{nm}^{2}\right)$ applied between the second Pt(II) ion and N7 of G19. Under such restraint, Pt(II) and N7 of G19 will get close to make the substitution easy to take place. For all the MD simulation a position restraint of $1.0 \times 10^{3} \mathrm{~kJ} /\left(\mathrm{mol} \cdot \mathrm{nm}^{2}\right)$ was applied to the heavy atoms of two bottom G-quartet (G5, G9, G14, G18, G4, G8, G13, G17) and bottom complex plane. The final structures of MD outcome were shown in figure 4-20. From the figures, the bottom plane of $\mathrm{Pt}(\mathrm{II})$ complex stacked well below the bottom G-quartet with $\mathrm{Pt}$ atom already coordinated to N7 of G2. Meanwhile, the top Pt(II) complex also became adjacent to G19. Such action made G19 leave the top G-quartet layer. The decomposition of top layer G-quartet also explained why only little increase was observed for $\Delta \mathrm{Tm}$ : the bottom $\mathrm{Pt}(\mathrm{II})$ complex may stabilize the G-quadruplex, while the bottom one may weaken such stabilization by induce the top G-quartet to decompose.
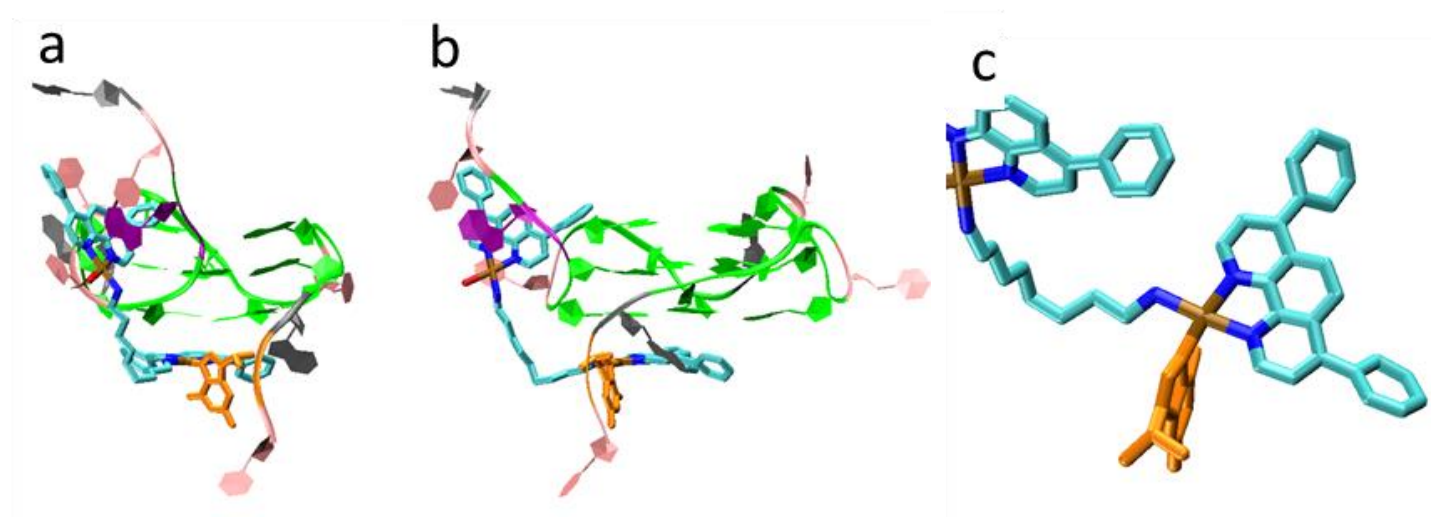

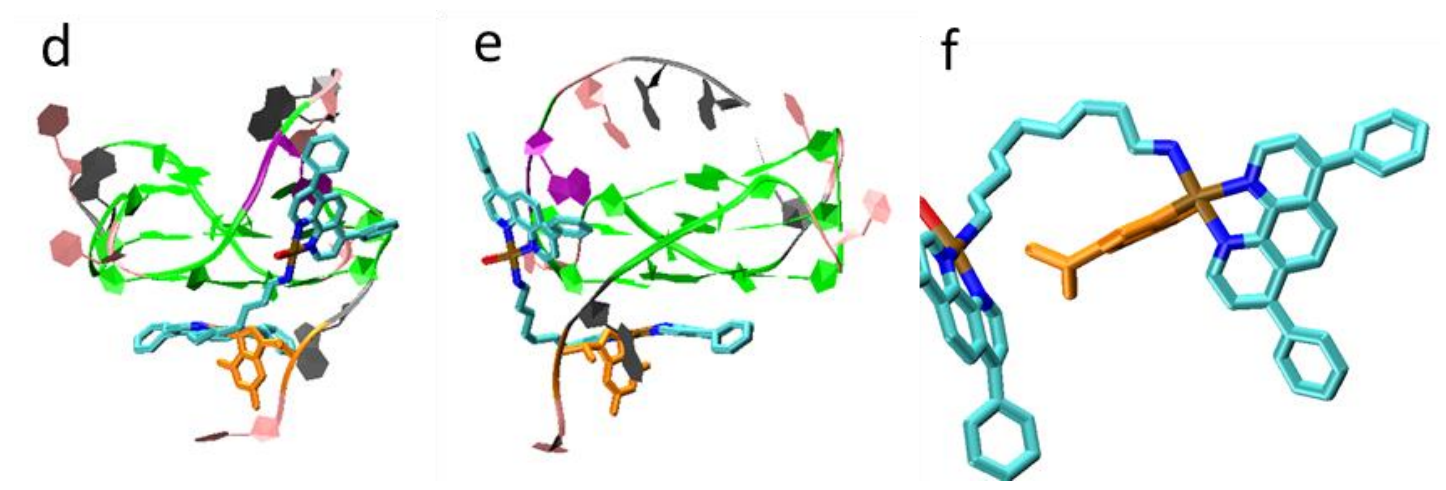

Figure 4-20 The structure of mono-substitued complex after 10 ns MD. For both conformations, the top Pt planes were close to G19 (a: conf1; d: conf2), another side view showed the top G-quartet has already decomposed under restraint (b: conf1; e: conf2). Zoom in of bottom Pt-guanine complex showed that the planar coordination geometry has formed. (c: conf1; f: conf2). The Pt complex and coordinate guanine were represented using Licorise model (Pt: brown; N: blue; C: cyan; Cl: red; guanine: orange), and the rest G-quadruplex was represented by new ribbon model (T: pink; A: gray; G: green; G2: orange; G19: purple).

\subsubsection{Constructing double-substituted product}

The second Pt(II) core and N7 of G19 were connected with the same procedure as we treated $\mathrm{Pt}(\mathrm{II})$ and $\mathrm{G} 2$. The obtained structure was then subject to MD simulation using GROMACS v4.6 package. Before MD simulation, the force field for double-substituted Pt-guanosine was built up by adding/replacing the Pt-containing terms based on the above mentioned parameters. With the updated force field, a new series of minimization, NVT (298K, $100 \mathrm{ps})$, NPT (1 atm, $100 \mathrm{ps)} \mathrm{equilibrium} \mathrm{were}$ conducted. As followed, REMD (replica exchange molecular dynamics) 
simulations $^{45,46}$ were conducted for both conformations to explore a more stable product structure.

The basic idea of REMD is to enhance sampling by heating up the system and then exchanged down the newly explored conformation to the lowest temperature. Several replicas with increasing temperatures were conducted synchronously. When the system energy of higher temperature replica is less than that of its lower temperature neighbor, then the coordinates and velocity of two replicas are exchanged. The temperature of each replica was set so that certain amplitude of exchange probability is maintained.

In our simulation, to save the computational resource, we conduct a 40ns REMD with 16 replicas. The temperature for each replica was set according to the website ${ }^{47}$ with a 0.2 exchange probability between neighboring replicas. The temperature range spanned from $298 \mathrm{~K}$ to $331 \mathrm{~K}$. The REMD simulation was conducted using GROMACS $\vee 5.1$ package $^{48}$. The position restraint for two bottom G-quartets and bottom complex plane was still applied. The trajectory at $298 \mathrm{~K}$ was then clustered using single-linkage method with a cutoff of $0.2 \mathrm{~nm}$ and the central structure of largest cluster was visualized by VMD. The visualization results were shown in figure 4-21. From the structure of crosslinked product, the different effect of two conformations to enzyme digestion could be illustrated: for conf1, the top $\operatorname{Pt}(\mathrm{II})$ attacked G19 across the backbone, the distance limit of alkyl linker required more movement of terminal bases (AATG) to meet the Pt(II). At last the terminal bases completely left G-quartet region and moved freely. Such conformation made the 
3'-end of DNA strand more vulnerable towards enzyme hydrolysis. On the contrary, for conf 2 the formation of top Pt(II)-guanosine complex required less movement of terminal bases, which still stay above G-quartet after substitution. Because of the bulky G-quartet, it was hard for enzyme to approach terminal bases. Hence incomplete digestion product from 3'-end may be observed by MS analysis.

a

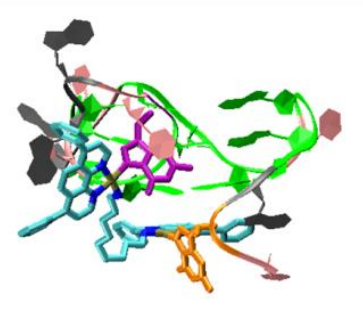

d

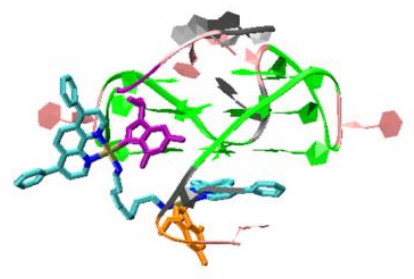

b

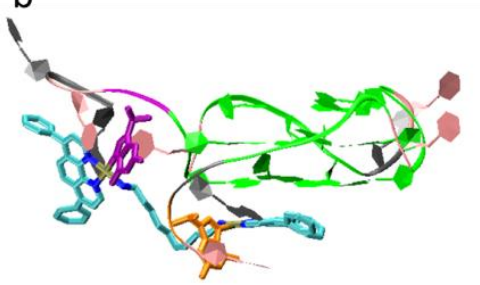

e

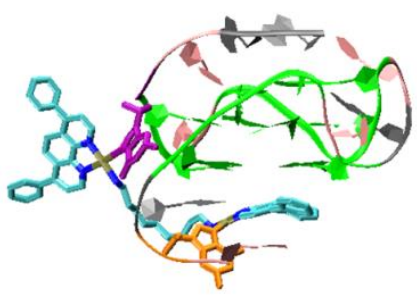

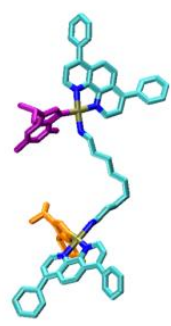

f

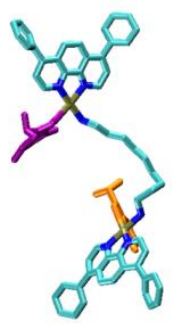

Figure 4-21 The structure of double-substituted complex after 40 ns REMD. For both conf1 (a) and conf2 (d) the top and bottom Pt(II)-guanosine complex have formed. 3'-AATG in conf1 was more separated from the G-quartet (b); while for conf2 that was close to its original position. Zoom in of Pt-guanine complex confirmed the planar coordination geometry. (c: conf1; f: conf2). The Pt complex and coordinate guanines were represented using Licorise model (Pt: brown; N: blue; C: cyan; Cl: red; G2: orange; G19: purple), and the rest G-quadruplex was represented by ribbon model (T: pink; A: gray; G: green; G2: orange; G19: purple).

In conclusion, our simulation work constructed the possible structure for dinuclear 
Pt(II)-guanosine complex by building up the customized force field. Distinct conformation of bis-crosslinked Pt3-G4 complexes were identified via tuning the initial docking poses.

\subsection{Conclusion}

Metal complex showed its power as a stabilizer as well as selector for different types of G-quadruplexes. In this chapter, series theoretical works have been done to demonstrate the experimental results. In section 4.2 and 4.3 by docking simulations the selectivity of metal complexes was illustrated. In section 4.4 the structure of dinuclear Pt(II) crosslink complex was explored by docking and MD simulation. The theoretical study provided a deeper understanding of the experimental phenomenon.

Publication information

Section 4.2 has been published on Journal of Inorganic Biochemistry, 2017, 166, 135.

DOI: $10.1016 /$ j.jinorgbio.2016.11.011

Section 4.3 has been published on Chem. Commun., 2016,52, 8095. DOI: 10.1039/C6CC03117J 


\section{Reference}

(1) Rhodes, D.; Lipps, H. J. G-quadruplexes and their regulatory roles in biology. Nucleic Acids Res 2015, 43 (18), 8627.

(2) Kim, N. W.; Piatyszek, M. A.; Prowse, K. R.; Harley, C. B.; West, M. D.; Ho, P. L. C.; Coviello, G. M.; Wright, W. E.; Weinrich, S. L.; Shay, J. W. Specific Association of Human Telomerase Activity with Immortal Cells and Cancer. Science 1994, 266 (5193), 2011.

(3) Blasco, M. A. Mammalian telomeres and telomerase: why they matter for cancer and aging. Eur J Cell Biol 2003, 82 (9), 441.

(4) Gellert, M.; Lipsett, M. N.; Davies, D. R. Helix Formation by Guanylic Acid. P Natl Acad Sci USA 1962, 48 (12), 2013.

(5) Yaku, H.; Fujimoto, T.; Murashima, T.; Miyoshi, D.; Sugimoto, N. Phthalocyanines: a new class of G-quadruplex-ligands with many potential applications. Chem Commun 2012, 48 (50), 6203.

(6) Han, F. X. G.; Wheelhouse, R. T.; Hurley, L. H. Interactions of TMPyP4 and TMPyP2 with quadruplex DNA. Structural basis for the differential effects on telomerase inhibition. $J$ Am Chem Soc 1999, 121 (15), 3561.

(7) Monchaud, D.; Teulade-Fichou, M. P. A hitchhiker's guide to G-quadruplex ligands. Org Biomol Chem 2008, 6 (4), 627.

(8) Collie, G. W.; Promontorio, R.; Hampel, S. M.; Micco, M.; Neidle, S.; Parkinson, G. N. Structural Basis for Telomeric G-Quadruplex Targeting by Naphthalene Diimide Ligands. $J$ Am Chem Soc 2012, 134 (5), 2723.

(9) Ou, T. M.; Lu, Y. J.; Tan, J. H.; Huang, Z. S.; Wong, K. Y.; Gu, L. Q. G-quadruplexes: Targets in anticancer drug design. Chemmedchem 2008, 3 (5), 690.

(10) Izbicka, E.; Wheelhouse, R. T.; Raymond, E.; Davidson, K. K.; Lawrence, R. A.; Sun, D. Y.; Windle, B. E.; Hurley, L. H.; Von Hoff, D. D. Effects of cationic porphyrins as G-quadruplex interactive agents in human tumor cells. Cancer Res 1999, 59 (3), 639.

(11) Shi, D. F.; Wheelhouse, R. T.; Sun, D. Y.; Hurley, L. H. Quadruplex-interactive agents as telomerase inhibitors: Synthesis of porphyrins and structure-activity relationship for the inhibition of telomerase. J Med Chem 2001, 44 (26), 4509.

(12) Reed, J. E.; Arnal, A. A.; Neidle, S.; Vilar, R. Stabilization of G-quadruplex DNA and inhibition of telomerase activity by square-planar nickel(II) complexes. J Am Chem Soc 2006, 128 (18), 5992.

(13) Bertrand, H.; Monchaud, D.; De Cian, A.; Guillot, R.; Mergny, J. L.; Teulade-Fichou, M. P. The importance of metal geometry in the recognition of G-quadruplex-DNA by metal-terpyridine complexes. Org Biomol Chem 2007, 5 (16), 2555.

(14) Talib, J.; Green, C.; Davis, K. J.; Urathamakul, T.; Beck, J. L.; Aldrich-Wright, J. R.; Ralph, S. F. A comparison of the binding of metal complexes to duplex and quadruplex DNA. Dalton T 2008, DOI:10.1039/b715458e 10.1039/b715458e(8), 1018.

(15) Redon, S.; Bombard, S.; Elizondo-Riojas, M. A.; Chottard, J. C. Platinum cross-linking of adenines and guanines on the quadruplex structures of the $\mathrm{AG}(3)(\mathrm{T}(2) \mathrm{AG}(3))(3)$ and ( $\mathrm{T}(2) \mathrm{AG}(3))(4)$ human telomere sequences in $\mathrm{Na}+$ and $\mathrm{K}+$ solutions. Nucleic Acids Res 2003, $31(6), 1605$.

(16) Ourliac-Garnier, I.; Elizondo-Riojas, M. A.; Redon, S.; Farrell, N. P.; Bombard, S. Cross-links 
of quadruplex structures from human telomeric DNA by dinuclear platinum complexes show the flexibility of both structures. Biochemistry-Us 2005, 44 (31), 10620.

(17) Redon, S.; Bombard, S.; Elizondo-Riojas, M. A.; Chottard, J. C. Platination of the $(\mathrm{T}(2) \mathrm{G}(4))(4)$ telomeric sequence: A structural and cross-linking study. Biochemistry-Us 2001, 40 (29), 8463.

(18) Bertrand, H.; Bombard, S.; Monchaud, D.; Teulade-Fichou, M. P. A platinum-quinacridine hybrid as a G-quadruplex ligand. J Biol Inorg Chem 2007, 12 (7), 1003.

(19) Viglasky, V. Platination of telomeric sequences and nuclease hypersensitive elements of human c-myc and PDGF-A promoters and their ability to form G-quadruplexes. Febs $J \mathbf{2 0 0 9}$, 276 (2), 401.

(20) Georgiades, S. N.; Abd Karim, N. H.; Suntharalingam, K.; Vilar, R. Interaction of Metal Complexes with G-Quadruplex DNA. Angew Chem Int Edit 2010, 49 (24), 4020.

(21) Read, M.; Harrison, R. J.; Romagnoli, B.; Tanious, F. A.; Gowan, S. H.; Reszka, A. P.; Wilson, W. D.; Kelland, L. R.; Neidle, S. Structure-based design of selective and potent G quadruplex-mediated telomerase inhibitors. P Natl Acad Sci USA 2001, 98 (9), 4844.

(22) Kim, M. Y.; Vankayalapati, H.; Kazuo, S.; Wierzba, K.; Hurley, L. H. Telomestatin, a potent telomerase inhibitor that interacts quite specifically with the human telomeric intramolecular G-quadruplex. J Am Chem Soc 2002, 124 (10), 2098.

(23) Ang, D. L.; Harper, B. W. J.; Cubo, L.; Mendoza, O.; Vilar, R.; Aldrich-Wright, J. Quadruplex DNA-Stabilising Dinuclear Platinum(II) Terpyridine Complexes with Flexible Linkers. Chem-Eur J 2016, 22 (7), 2317.

(24) Luo, D.; Mu, Y. G. All-Atomic Simulations on Human Telomeric G-Quadruplex DNA Binding with Thioflavin T. J Phys Chem B 2015, 119 (15), 4955.

(25) Lape, M.; Elam, C.; Paula, S. Comparison of current docking tools for the simulation of inhibitor binding by the transmembrane domain of the sarco/endoplasmic reticulum calcium ATPase. Biophys Chem 2010, 150 (1-3), 88.

(26) Dennington, R.; Keith, T.; Millam, J.; Semichem Inc., Shawnee Mission, KS, 2009.

(27) Frisch, M. J.; Trucks, G. W.; Schlegel, H. B.; Scuseria, G. E.; Robb, M. A.; Cheeseman, J. R.; Scalmani, G.; Barone, V.; Mennucci, B.; Petersson, G. A.et al.; Gaussian, Inc.: Wallingford, CT, USA, 2009.

(28) Jones, G.; Willett, P.; Glen, R. C. Molecular Recognition of Receptor-Sites Using a Genetic Algorithm with a Description of Desolvation. J Mol Biol 1995, 245 (1), 43.

(29) Jones, G.; Willett, P.; Glen, R. C.; Leach, A. R.; Taylor, R. Development and validation of a genetic algorithm for flexible docking. J Mol Biol 1997, 267 (3), 727.

(30) Humphrey, W.; Dalke, A.; Schulten, K. VMD: Visual molecular dynamics. J Mol Graph Model 1996, 14 (1), 33.

(31) Rajput, C.; Rutkaite, R.; Swanson, L.; Haq, I.; Thomas, J. A. Dinuclear monointercalating $\mathrm{Ru}-\mathrm{II}$ complexes that display high affinity binding to duplex and quadruplex DNA. Chem-Eur J 2006, 12 (17), 4611.

(32) Shi, S.; Liu, J.; Yao, T. M.; Geng, X. T.; Jiang, L. F.; Yang, Q. Y.; Cheng, L.; Ji, L. N. Promoting the formation and stabilization of G-quadruplex by dinuclear Ru-II complex Ru-2(obip)L-4. Inorg Chem 2008, 47 (8), 2910.

(33) Xu, L.; Chen, X.; Wu, J. H.; Wang, J. Q.; Ji, L. N.; Chao, H. Dinuclear Ruthenium(II) Complexes That Induce and Stabilise G-Quadruplex DNA. Chem-Eur J 2015, 21 (10), 4008. 
(34) Ma, D. L.; Che, C. M.; Yan, S. C. Platinum(II) Complexes with Dipyridophenazine Ligands as Human Telomerase Inhibitors and Luminescent Probes for G-Quadruplex DNA. J Am Chem Soc 2009, 131 (5), 1835.

(35) Ou, Z. Z.; Feng, Z.; Liu, G. X.; Chen, Y. J.; Gao, Y. Y.; Li, Y.; Wang, X. S. Synthesis and G-Quadruplex-binding Properties of Cationic Platinum(II) Terpyridine Complexes Containing sigma-Alkynyl Auxiliaries. Chem Lett 2015, 44 (4), 425.

(36) Kieltyka, R.; Fakhoury, J.; Moitessier, N.; Sleiman, H. F. Platinum phenanthroimidazole complexes as G-quadruplex DNA selective binders. Chem-Eur J 2008, 14 (4), 1145.

(37) Li, P. F.; Merz, K. M. MCPB.py: A Python Based Metal Center Parameter Builder. J Chem Inf Model 2016, 56 (4), 599.

(38) Wang, J. M.; Wolf, R. M.; Caldwell, J. W.; Kollman, P. A.; Case, D. A. Development and testing of a general amber force field. J Comput Chem 2004, 25 (9), 1157.

(39) Seminario, J. M. Calculation of intramolecular force fields from second-derivative tensors. Int J Quantum Chem 1996, 60 (7), 1271.

(40) Bayly, C. I.; Cieplak, P.; Cornell, W. D.; Kollman, P. A. A Well-Behaved Electrostatic Potential Based Method Using Charge Restraints for Deriving Atomic Charges - the Resp Model. J Phys Chem-Us 1993, 97 (40), 10269.

(41) Besler, B. H.; Merz, K. M.; Kollman, P. A. Atomic Charges Derived from Semiempirical Methods. J Comput Chem 1990, 11 (4), 431.

(42) Li, P. F.; Roberts, B. P.; Chakravorty, D. K.; Merz, K. M. Rational Design of Particle Mesh Ewald Compatible Lennard-Jones Parameters for +2 Metal Cations in Explicit Solvent. $J$ Chem Theory Comput 2013, 9 (6), 2733.

(43) Yao, S. J.; Plastaras, J. P.; Marzilli, L. G. A Molecular Mechanics Amber-Type Force-Field for Modeling Platinum Complexes of Guanine Derivatives. Inorg Chem 1994, 33 (26), 6061.

(44) Dassault Systèmes BIOVIA, D. S. M. E., Release 2017, San Diego: Dassault Systèmes, 2016.

(45) Sugita, Y.; Okamoto, Y. Replica-exchange molecular dynamics method for protein folding. Chem Phys Lett 1999, 314 (1-2), 141.

(46) Nadler, W.; Hansmann, U. H. E. Dynamics and optimal number of replicas in parallel tempering simulations. Phys Rev E 2007, 76 (6).

(47) Patriksson, A.; van der Spoel, D. A temperature predictor for parallel tempering simulations. Phys Chem Chem Phys 2008, 10 (15), 2073.

(48) Abraham, M. J.; Murtola, T.; Schulz, R.; Páll, S.; Smith, J. C.; Hess, B.; Lindahl, E. GROMACS: High performance molecular simulations through multi-level parallelism from laptops to supercomputers. SoftwareX 2015, $1-2,19$. 


\section{Appendix}

Using Amino-Labeled Nucleotide Probes for

Simultaneous Single Molecule RNA-DNA FISH 


\section{Introduction}

\subsection{The progress of FISH}

Because of the crucial function of nucleic acid in various biological processes, to visualize or trace the position of DNA or RNA in vivo is always demanding. But the difficulty is as expected: the routine clinical approaches, such as optical microscopy, X-ray et al. , are unable to detect a nanometer-scale target at physiological condition. Under such circumstances, in situ hybridization (ISH), based on the fundamental property of complementary paring of nucleic acid ${ }^{1}$, provided an excellent solution to the problem: a probe, usually DNA, labeled with some signaling group, has the complementary sequence of the target sequence and thus can pair with the target. The target needn't to be extracted and run post analysis; instead its position is studied in its original position (Figure 1). Original ISH use radionuclide labeled probe to visualize the target, however, the shortcomings of its deadly radioactivity made it unacceptable for clinical application. Based on such point, a milder signaling group-fluorophore -was introduced and such method is called fluorescent in situ hybridization (FISH) ${ }^{2}$. Compared with ISH, the advantage of FISH is apparent: the biocompatibility makes it available for mass application in cytogenetic laboratory; besides, the diversity of fluorophore can be used to detect different kinds of targets in one experiment.

The early stage of FISH application saw the usage of RNA directly linked with fluorophore $^{3}$ or multi-labeled probe using nick-translation ${ }^{4}$. The application of amino-conjugated $^{5}$ probe made it a simple way to produce low-noise probe. 
Immunofluorescence technology ${ }^{6}$ improve the specificity greatly and synthetic DNA probe $^{7}$ carrying enough fluorophore made direct detection of the target sequence available. In recent years, suppression hybridization ${ }^{8}$ was proposed to overcome the non-specific hybridization of probe. And the application of FISH to chromosome analysis also achieved pronounced progress ${ }^{9}$. It's now feasible to visualize all human chromosomes with different colors simultaneously with the help of computational techniques $^{10,11}$. At the same time, the effort to reduce the probe size led to higher signal-to-noise ratio $^{12}$.

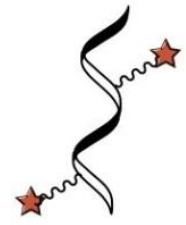

Labeled probe

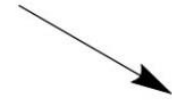

(1)

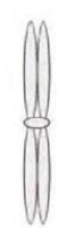

Chromosomal target

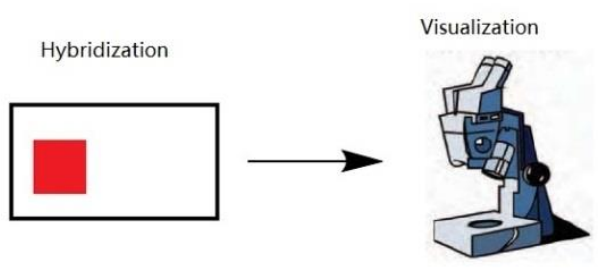

Figure 1 The scheme of FISH.

\subsection{Labeling types}

Based on the different types of labelling method, ususlly there are two major methods to link the fluorophore, such as fluorescein, rhodamine or coumarin, to the probe: direct FISH and indirect FISH ${ }^{13}$. As shown in figure 2, in direct FISH the probes are directly labelled by above-mentioned fluorophores. Usually two typical methods are used to link the fluorophores to the probes: the first method is to use synthetic method to directly combine the fluorophore molecule onto the DNA probe 
sequences. The second one, also referred as nick translation, is to link a fluorescent label into a nucleoside triphosphate and then use enzymes to insert the above nucleoside triphosphate into the DNA probe sequences.

While for the indirect FISH, the probe is labeled by hapten, such as biotin and digoxigenin, and then immunofluorescence technique is used to detect the signal. Compared with indirect FISH, the direct FISH is much faster and causes less damage to the target nucleotide sequence, but its sensitivity is less than indirect $\mathrm{FISH}^{14}$.

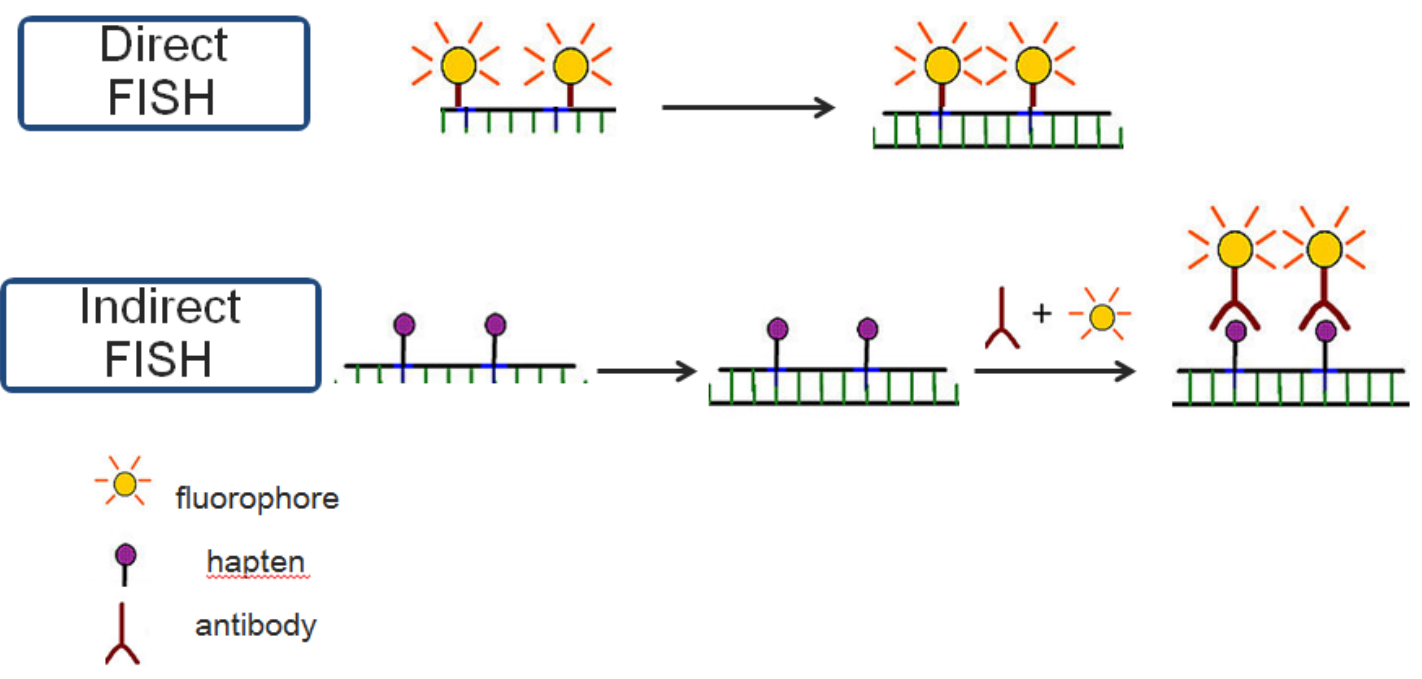

Figure 2 The scheme of direct and indirect FISH

The visualization sometimes still needs to be accomplished using a procedure called counterstain ${ }^{13}$ which is a stain with colour contrasting to the principal stain, making the stained structure more easily visible. The most common counterstains used in FISH are propidium iodide and diamidino-2-phenylindole(DAPI).

\subsection{Simultaneous RNA-DNA FISH}

Many important cellular biological processes involve the participation of RNA 
and DNA together. For example, investigating the interaction between long non-coding RNA (lncRNA) and their DNA targets is popular because many of lncRNAs play crucial roles in nuclear architecture and epigenetic regulation ${ }^{15}$.

However, to visualize RNA and DNA simultaneously was hard in practice, since harsh condition to denature the DNA will destroy RNA or RNA FISH signal. How to visualize DNA target and protect the RNA FISH signal at the same time is a key question in this field. The previous experimental studies can be divided into 3 categories ${ }^{16}$. The first one is the simultaneous hybridization approach ${ }^{17}$, in which RNA and DNA FISH are conducted at the same time. As the problem mentioned above, the application of such method is very limited. The second one is the superimposition approach ${ }^{18}$. In this approach, RNA FISH was carried out and the image was collected firstly; then the DNA FISH was conducted and the collected image was superimposed in the same coordinate system as the one in the RNA FISH image so that the two FISH images are overlapped as if they are simultaneous taken. Actually this method is not a real simultaneous RNA-DNA FISH. The third approach, the so-called post fixation method, tries to fix the RNA FISH signal to make it survive the subsequent DNA FISH. Zhang L.F. et al. reported some successful studies using such method ${ }^{16}$. In these studies, they employed indirect immunostain for RNA FISH, after that, formaldehyde was used to fix the fluorophore groups to their proximal protein so that RNA FISH signal can survive the denaturation step of DNA FISH. They applied their method to visualize Xist (inactivated X chromosome specific transcript) and corresponding Xic (X chromosome inactivation center), as well as 
Terra (telomeric repeat-containing RNA) and telomere DNA simultaneously, the result is satisfactory.

However, it should be pointed out that such simultaneous RNA-DNA FISH method has obvious shortcomings: the multiple steps of immunostain made it complicated and expensive. What's more, indirect RNA FISH dumped the signal-to-noise ratio thus made subsequent DNA FISH signal hard to detect. To overcome such difficulties, a robust, direct RNA-DNA FISH method is proposed, in which amino-labeled probe was used to avoid the use of immunostain technology in RNA FISH. In the later sections, the principle and application of this method will be discussed in detail.

\section{Methods}

\subsection{Basic principle}

The crosslink of amino groups using formaldehyde is the key to post fixation step. Actually, formaldehyde is a commonly used reagent for crosslink reaction in biological experiment long time ago ${ }^{19}$. While in previous study, the amino group was usually provided by lysine residue from protein $^{20}$ and that explains why immunostain was necessary for RNA-DNA FISH. Here we propose a novel strategy to introduce amino group directly into probe, either by synthesis or nick translation. Under such condition, the tedious immunostain step can be skipped (figure 3). Based on such design, the amino-labeled DNA probe was firstly synthesized and then applied to several systems to prove its feasibility. 
A

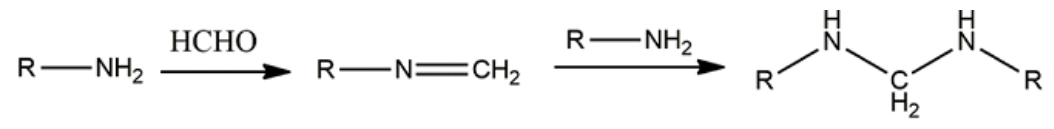

B

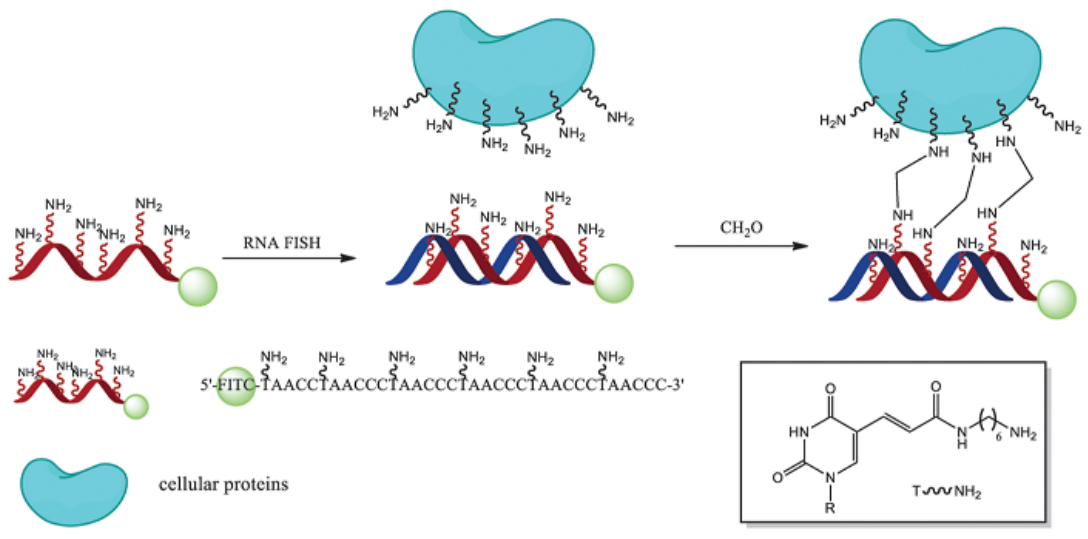

Figure 3 A: the reaction of post-fixation using formaldehyde. B: The scheme of direct RNA-DNA FISH

\subsection{Preparetion of amino-labeled oligonucleotide probes}

Two different types of fluorophores, FAM and Cy5, were introduced into the probe for different targets. For FAM-labeled amino probe synthesis, all the normal and amino-dT phosphoramidite were purchased from Glen Research (USA) and coupled on an automated DNA synthesizer (Mermade 4, Bioautomation, USA). The standard coupling was applied for all the phosphoramidite incorporation. Thereafter the FAM phosphoramidite was coupled to the 5' of the oligonucleotide probe using solid phase phosphoramidite chemistry. All the protection group were then deprotected by AMA at ambient temperature and then subjected to HPLC (Shimadzu, C18 column, $0 \%-15 \%$ ACN over $50 \mathrm{mM}$ triethylammonium acetate buffer, at $\mathrm{pH} 7.0$ for $30 \mathrm{~min}$ ) for purification.

An oligonucleotide probe set with Cy5 label was designed according to Stellaris Probe Designer (https://www.biosearchtech.com/) to target EGFP mRNA. Since no 
Cy5-decorated phosphoramidite was available commercially, the fluorophore is designed to be coupled to the probe afterwards. First of all, the oligonucleotides with or without amino-dT were synthesized and a C6-alkyl amino linker was introduced into the 5' of the DNA. Then Cy5-NHS ester (Cat\# 23020, Lumiprobe, USA) was used and coupled to the amino terminal in the presence of 10 eq of DIPEA in anhydrous DMF for 2 hours at room temperature. It should be noted that up to now the amino-dT was still under protection so the Cy5 can only be coupled to the terminal C6-amino. Then the modified oligonucleotide were cleaved from resin beads and deprotected in saturated ammonium hydroxide solution simultaneously for 12 hours at $60^{\circ} \mathrm{C}$, followed by HPLC purification. The HPLC absorption peaks at 260nm and 640nm were collected to confirm the successful coupling of Cy5 onto DNA probe. All the fluorophore coupled DNA probes were confirmed using ESI-MS (Sangon, China).

The Telomere DNA FISH probes were prepared using nick translation kit (Roche, Cat\# 10976776001). Nucleotide analog used in probe labeling was Cy3-dUTP (Amersham, Cat\# PA53022). Besides, a FAM-labeled PNA probe (Panagene, Cat\# F1009) was used as a control in Terra RNA FISH.

\subsection{Electrophoretic mobility shift assay}

The probe and target (telomere) duplex were subject to both $20 \%$ native PAGE and $15 \%$ denature PAGE with or without Bovine serum albumin (Amersco, USA) before and after post-fixation. The condition for native and denature PAGE is $7 \mathrm{~V}$ for 7 hours and $10 \mathrm{~V}$ for 4hours, respectively. The gel was then visualized using 
phosphor-imager (Typhoon Trio, GE).

\subsection{RNA and DNA FISH}

A transformed female mouse embryonic fibroblast (MEF) cell line ${ }^{18}$, a human breast cancer cell line (HCC 1937)(ATCC) and a mouse embryonic stem cell line (J1)(ATCC) were cultured in DMEM medium with $10 \% \mathrm{FBS}$ at $37^{\circ} \mathrm{C}$ in a $5 \% \mathrm{CO}_{2}$ incubator.

Cells were harvested, fixed and permeabilized before FISH. For RNA FISH, slides were dehydrated, dried and hybridized with probes for 3 hours at $42^{\circ} \mathrm{C}$ in a dark and humid condition. After that, slides were post-fixed in $4 \%$ PFA(paraformaldehyde) and rinsed with PBS. Then $70 \%$ formamide, $2 x$ SSC was used to denature DNA target at $80^{\circ} \mathrm{C}$ for $10 \mathrm{~min}$. The slides were dehydrated and probes were applied for an overnight hybridization at $42^{\circ} \mathrm{C}$ in a dark and humid condition.

\subsection{Microscopy and data analysis}

Eclipse Ti microscope (Nikon) was used to collect the Fluorescence images using digital camera Clara Series model C01 (Andor) and deconvoluted using NIS Element AR imaging software (Nikon).

To measure individual telomere DNA FISH signal intensity, images for multiple z-section of a same slide were collected using the same exposure time. The images from the same z-section were merged and the sum fluorescent intensity of each telomere DNA FISH signal was measured using NIS Element AR imaging software 
(Nikon).

To measure the surviving signal intensity of EGFP mRNA signal, the total RNA signal per cell was measured from the Cy5 channel. The EGFP protein fluorescent signal was used to mark the cell boundary. All fluorescent images from the two slides (the amino-labeled set and the regular set) were collected using the same exposure time for each fluorescent channel. The total EGFP fluorescent signal was also taken to normalize the experimental variation between two slides as well as the different cells within the same slide.

\section{Results and discussion}

\subsection{Characterization of synthetic amino-oligonucleotide}

Terra is a repeat containing lncRNA transcribed from telomeric DNA region. The probe sequence for Terra is (TAACCC) 6 . As introduced before, an amino group was connected to each dT by an alkyl group and a fluorophore was coupled to the 5 ' of the probe. The dual-label of the probe was confirmed using UV absorption spectrum when conducting HPLC purification and further ESI-MS spectrum, as figure 4 shows.

The crosslink reaction was firstly studied by PAGE (figure 5). Because of the repeat sequence of the probe, up to 6 amino groups was introduced to make the stable crosslink product. No crosslink reaction occurred when BSA is not present (lane 3 and lane 7 in figure 4). It was further confirmed that covalently crosslink product can only form when formaldehyde was present: the upper band in lane 2 disappeared in denature protocol. Besides, from lane 8 it can be seen that the long linkage of 
amino-probe makes its amino groups approached farther amino of the protein. The long smear band up in the lane 8 showed a larger product was formed, in which the amino groups of the probe extended to react with some non-bound protein in the vicinal area.
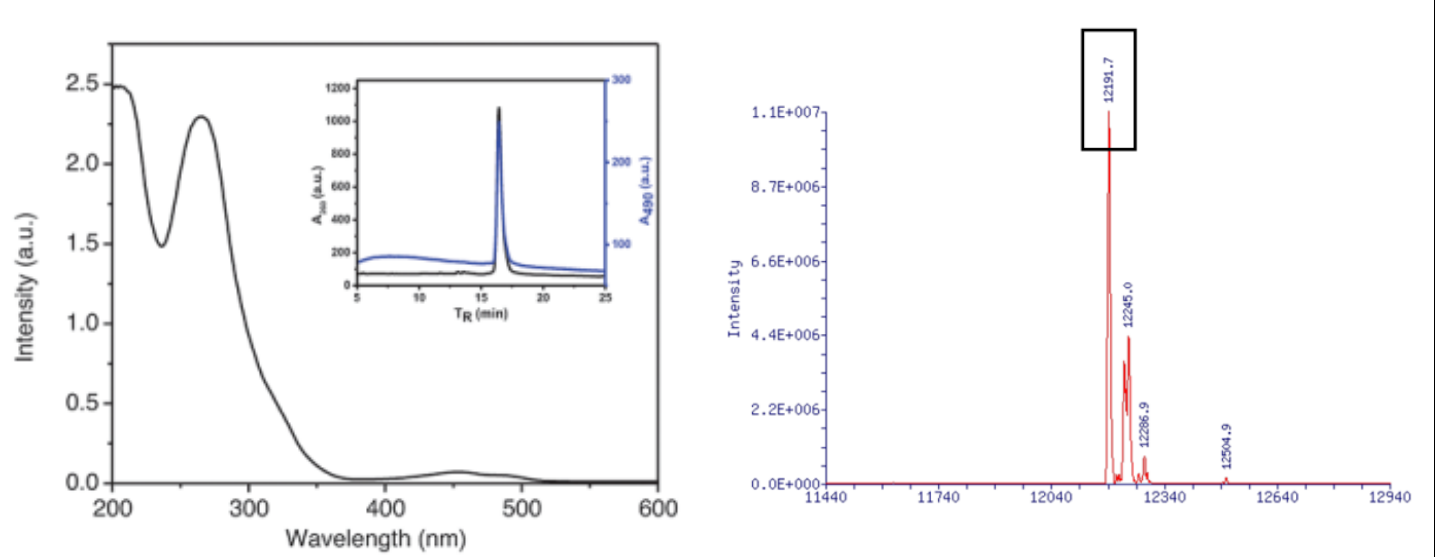

Figure 4 Left: The UV-spectrum of the Terra probe, Insert: the HPLC purification trace of the dual-labeled probe. Right: ESI-MS spectrum of the Terra probe, the calculated MW is 12191.

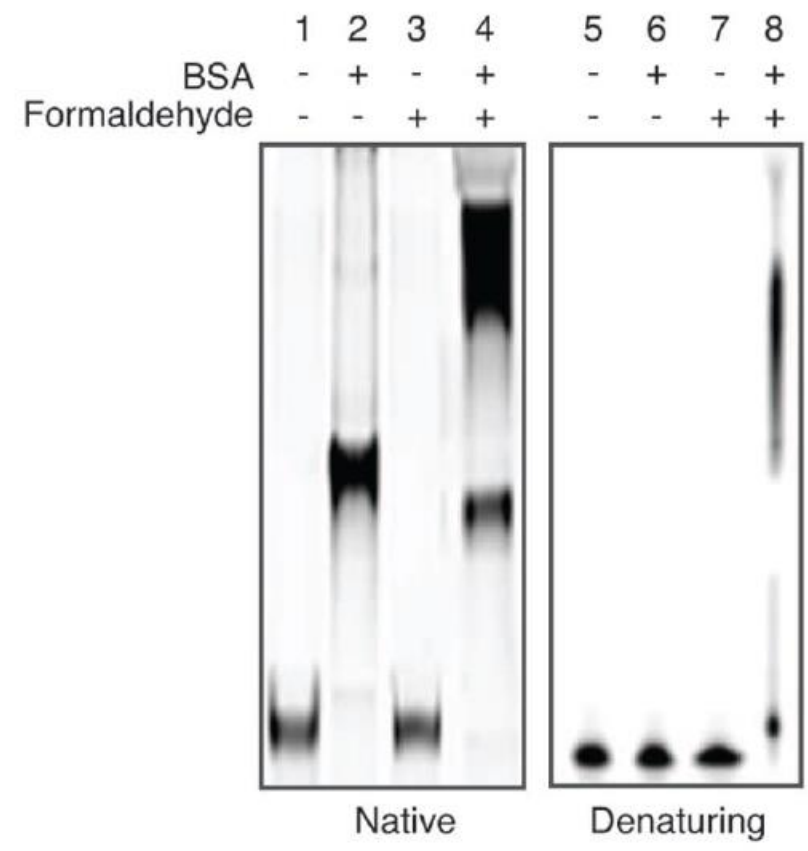

Figure 5 Electrophoretic mobility shift assay of crosslink between amino-probe and 
bovine serum albumin (BSA) on native (lane 1-4) and denature PAGE (lane 5-8)

\subsection{Simultaneous detection of Terra and telomere DNA}

The above amino-probe with FAM label was then used for RNA FISH. A FAM-peptide nucleic acid (PNA) was used to act as the control because of its outstanding thermal and chemical stability. The result was shown in figure 6. From upper row of figure 6A, the RNA FISH signal was lost after DNA FISH despite the stability of PNA; on the contrary, when using dual-labeled oligonucleotide probe, the RNA FISH signal can survive well after DNA FISH, as shown in lower row of figure 6A, in which two brighter spots of RNA FISH signal can be detected. Besides, in each nucleus some RNA FISH signal were close to red FISH signal while some were not.

Such founding was supported by previous studies ${ }^{16,21}$. The RNA FISH signal size was also measured before and after DNA FISH, as shown in figure 6B, the RNA FISH signal shows almost consistent distribution, which indicate the RNA signal was well protected after DNA FISH.

The function of Terra is still under investigation, while it was hypothesized that Terra may act as a Telomere expression inhibition, the binding of Terra to corresponding Telomere region may restrict the association of Telomerase to such region. It was further hypothesized that Terra has more affinity to longer Telomere ${ }^{22}$, to test this, we measure the DNA FISH signal both associated and non-associated with Terra FISH signal, from figure $6 \mathrm{C}$, the associated DNA signal is slightly higher than non-associated one, but such small difference may not convincing enough and more accurate approach is still required. 
A
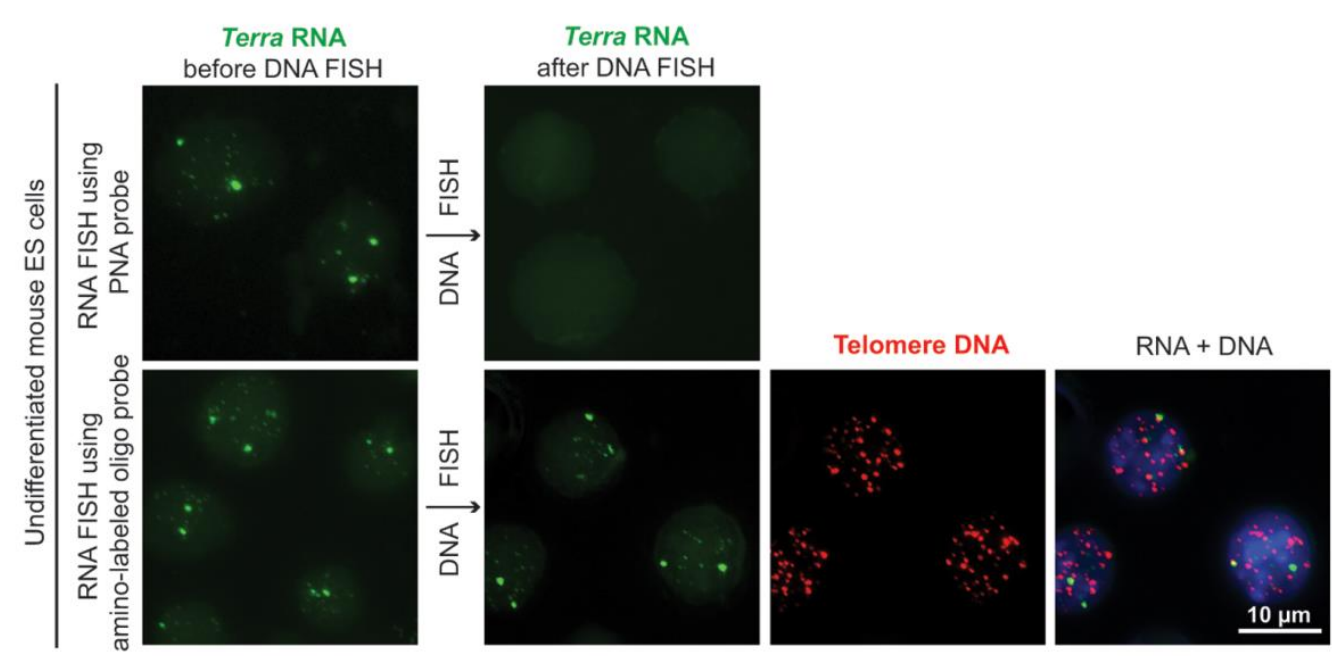

B

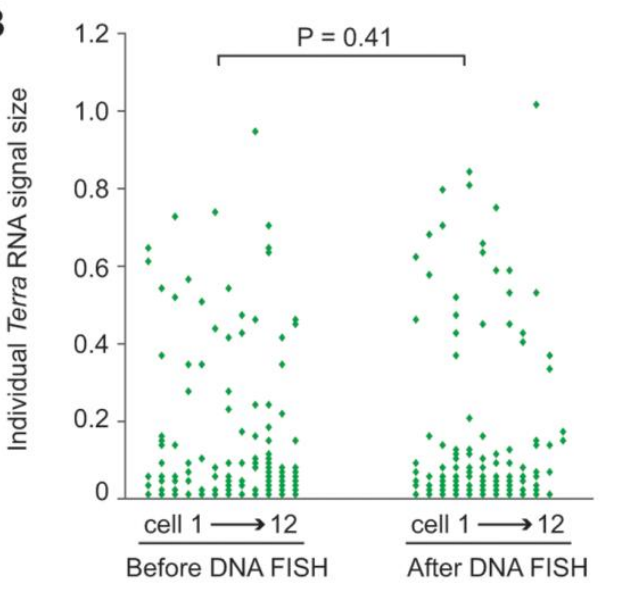

C

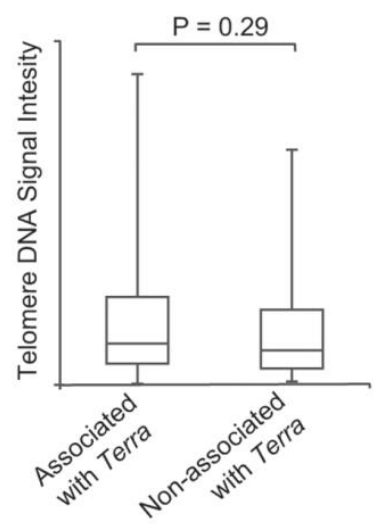

Figure 6 A: The RNA-DNA FISH image of Terra and telomere DNA. Either a PNA probe (upper row) or amino-oligonucleotide probe (lower row) was used. The green pinpoint came from FAM and red one came from Cy3. DAPI (blue) was used as counterstain. B: The Terra signal per nucleus before and after DNA FISH was measured. Individual Terra signal in each cell was recognized by setting a fluorescent threshold, the size of each Terra signal $\left(\mu \mathrm{m}^{2}\right)$ was calculated. C: DNA FISH signal intensity of telomere was measured to quantitate telomere length. P-value was computed using Student T-test.

\subsection{Simultaneous detection of single mRNA molecule with DNA}

With the success to visualize Terra and telomeric DNA simultaneously, we tried 
to further expand the application of it to single mRNA molecule FISH, which is more challenging because the sequence of mRNA is not repetitive. As a result, probes labeled with limited fluorophore will be too weak for signaling. To circumvent such problem, in previous study, a probe set which contains more than one labeled oligonucleotide was designed ${ }^{23}$. Here we adopted a similar method which uses more probes in the probe set to visualize the mRNA of enhanced green fluorescent protein (EGFP) in the cytoplasm of mouse fibroblast cells transiently transfected by an EGFP-expressing plasmid. According to the website, the sequence was listed as in table 1 . The synthetic probe was confirmed with the similar UV absorption of HPLC as well as the MS spectrum, one example of both methods will be shown in figure 7 .

Table 1 The designed probe set sequence for EGFP mRNA FISH

\begin{tabular}{|c|c|c|c|}
\hline 1 & GTCCAGCTCGACCAGGATGG & 11 & CGATGCCCTTCAGCTCGATG \\
\hline 2 & CTGAACTTGTGGCCGTTTAC & 12 & ATGTTGCCGTCCTCCTTGAA \\
\hline 3 & AGCTTGCCGTAGGTGGCATC & 13 & GTAGTTGTACTCCAGCTTGT \\
\hline 4 & GTGGTGCAGATGAACTTCAG & 14 & CATGATATAGACGTTGTGGC \\
\hline 6 & CACTGCACGCCGTAGGTCAG & 15 & ATGCCGTTCTTCTGCTTGTC \\
\hline 7 & TACTTGAAGAAGTCGTGCTG & 16 & GCGGATCTTGAAGTTCACCT \\
\hline 8 & CTTGAAGAAGATGGTGCGCT & 18 & TGTGATCGCGCTTCTCGTTG \\
\hline 9 & CGGGTCTTGTAGTTGCCGTC & 19 & TGTGATCGCGCTTCTCGTTG \\
\hline
\end{tabular}



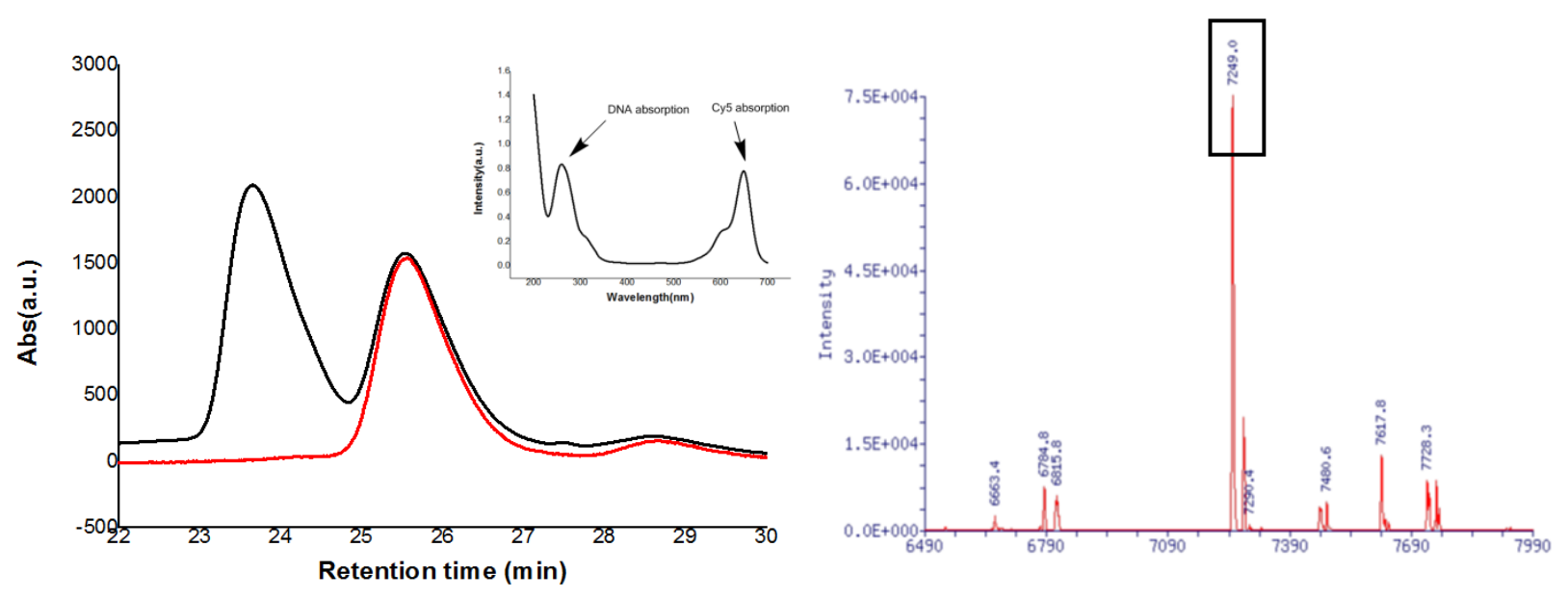

Figure 7 Left: The UV-spectrum of the mRNA probe, Insert: the HPLC purification trace of the probe. Right: ESI-MS spectrum of the probe, the calculated MW is 7250.

The probe set was then used in RNA FISH and the collected images were shown in figure 8. DAPI, as a counterstain reagent, was used to make the contrast clearer. GFP here was visualized to mark the boundary of the cell. In figure 8A, it can be seen that both regular probe set and amino-probe set can mark the mRNA properly, which appeared as the white pinpoint signal. However, in figure $8 \mathrm{~B}$, after DNA FISH the mRNA signal using regular probe set suffered severe damage, on the other hand the amino-labeled probe set can protect mRNA signal efficiently. The mRNA signals remained as discrete pinpoints and only appeared in the cytoplasm of the EGFP-expressing cells. Such difference can be illustrated more clearly in figure 8C, in which the mRNA signal intensity per cell was measured and normalized according to intensity of GFP. The dramatic increase of normalized intensity of mRNA signal supported that our strategy also worked well for mRNA FISH. 


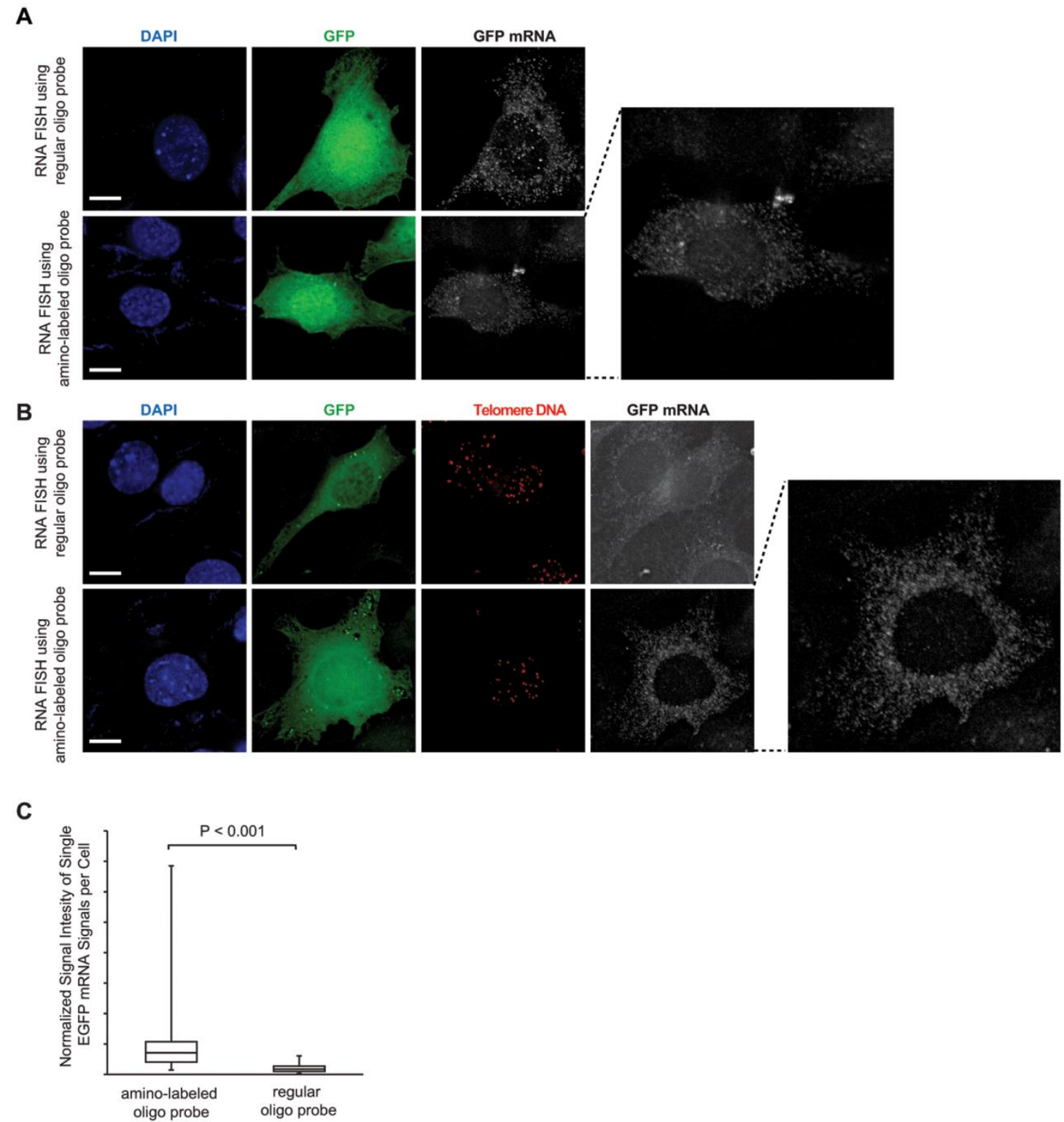

Figure 8 A: The RNA FISH image of EGFP mRNA only. Both regular probe and amino-probe were used. Cy5 signal was shown as white pinpoint. B: RNA-DNA FISH image of EGFP mRNA and telomere DNA. C: Normalized mRNA signal intensity per cell measured from Cy5 channel. P-value was computed using Student T-test.

\section{Conclusion}

The established method provides a robust way for simultaneous direct RNA-DNA 
FISH and can be used for long list of regulatory lncRNAs and their DNA targets in the nucleus. Besides, this method can also be used for clinical application, such as the direct observation of FGFR 1 gene expression at the single cell level ${ }^{24}$.

In conclusion, with dual-labeled oligonucleotide probe, we can visualize RNA and DNA target directly at the single-cell and single-molecule level. Such protocol can be further applied to the study of large pool of nuclear lncRNA

Publication information

This work has been published on the following journal/book:

1. Using Amino-Labeled Nucleotide Probes for Simultaneous Single Molecule RNA-DNA FISH, PLOS One, 2014, 9(9). DOI :10.1371/journal.pone.0107425

2. Simultaneous RNA-DNA FISH, in Long Non-Coding RNAs, pp 135-145, DOI: 10.1007/978-1-4939-3378-5_11 


\section{Reference}

(1) Pardue, M. L.; Gall, J. G. Molecular Hybridization of Radioactive DNA to DNA of Cytological Preparations. P Natl Acad Sci USA 1969, 64 (2), 600.

(2) Pinkel, D.; Gray, J. W.; Trask, B.; van den Engh, G.; Fuscoe, J.; van Dekken, H. Cytogenetic analysis by in situ hybridization with fluorescently labeled nucleic acid probes. Cold Spring Harb Symp Quant Biol 1986, 51 Pt 1, 151.

(3) Bauman, J. G. J.; Wiegant, J.; Borst, P.; Vanduijn, P. A New Method for Fluorescence Microscopical Localization of Specific DNA-Sequences by Insitu Hybridization of Fluorochrome-Labeled Rna. Exp Cell Res 1980, 128 (2), 485.

(4) Wiegant, J.; Ried, T.; Nederlof, P. M.; Vanderploeg, M.; Tanke, H. J.; Raap, A. K. Insitu Hybridization with Fluoresceinated DNA. Nucleic Acids Res 1991, 19 (12), 3237.

(5) Langer, P. R.; Waldrop, A. A.; Ward, D. C. Enzymatic-Synthesis of Biotin-Labeled Polynucleotides - Novel Nucleic-Acid Affinity Probes. P Natl Acad Sci-Biol 1981, 78 (11), 6633.

(6) Manuelidis, L.; Langersafer, P. R.; Ward, D. C. High-Resolution Mapping of Satellite DNA Using Biotin-Labeled DNA Probes. J Cell Biol 1982, 95 (2), 619.

(7) Kislauskis, E. H.; Li, Z. F.; Singer, R. H.; Taneja, K. L. Isoform-Specific 3'-Untranslated Sequences Sort Alpha-Cardiac and Beta-Cytoplasmic Actin Messenger-Rnas to Different Cytoplasmic Compartments. J Cell Biol 1993, 123 (1), 165.

(8) Landegent, J. E.; Dewal, N. J. I.; Dirks, R. W.; Baas, F.; Vanderploeg, M. Use of Whole Cosmid Cloned Genomic Sequences for Chromosomal Localization by Non-Radioactive Insitu Hybridization. Hum Genet 1987, 77 (4), 366.

(9) Trask, B. J. Human cytogenetics: 46 chromosomes, 46 years and counting. Nat Rev Genet 2002, 3 (10), 769.

(10) Schrock, E.; du Manoir, S.; Veldman, T.; Schoell, B.; Wienberg, J.; Ferguson-Smith, M. A.; Ning, Y.; Ledbetter, D. H.; Bar-Am, I.; Soenksen, D.et al. Multicolor spectral karyotyping of human chromosomes. Science 1996, 273 (5274), 494.

(11) Speicher, M. R.; Gwyn Ballard, S.; Ward, D. C. Karyotyping human chromosomes by combinatorial multi-fluor FISH. Nat Genet 1996, 12 (4), 368.

(12) Lawrence, J. B.; Singer, R. H.; Villnave, C. A.; Stein, J. L.; Stein, G. S. Intracellular distribution of histone mRNAs in human fibroblasts studied by in situ hybridization. Proc Natl Acad Sci U S A 1988, 85 (2), 463.

(13) Gersen, S. L.; Keagle, M. B. The principles of clinical cytogenetics; Humana Press: Totowa, NJ, 1999.

(14) Basu, R.; Lai, L. T.; Meng, Z. Y.; Wu, J.; Shao, F. W.; Zhang, L. F. Using Amino-Labeled Nucleotide Probes for Simultaneous Single Molecule RNA-DNA FISH. Plos One 2014, 9 (9).

(15) Wilusz, J. E.; Sunwoo, H.; Spector, D. L. Long noncoding RNAs: functional surprises from the RNA world. Genes Dev 2009, 23 (13), 1494.

(16) Lai, L. T.; Lee, P. J.; Zhang, L. F. Immunofluorescence protects RNA signals in simultaneous RNA-DNA FISH. Exp Cell Res 2013, 319 (3), 46.

(17) Chaumeil, J.; Le Baccon, P.; Wutz, A.; Heard, E. A novel role for Xist RNA in the formation of a repressive nuclear compartment into which genes are recruited when silenced. Gene Dev 2006, 20 (16), 2223. 
(18) Zhang, L. F.; Huynh, K. D.; Lee, J. T. Perinucleolar targeting of the inactive X during S phase: Evidence for a role in the maintenance of silencing. Cell 2007, 129 (4), 693.

(19) French, D.; Edsall, J. T. The Reactions of Formaldehyde with Amino Acids and Proteins. $A d v$ Protein Chem 1945, 2, 277.

(20) Fox, C. H.; Johnson, F. B.; Whiting, J.; Roller, P. P. Formaldehyde Fixation. J Histochem Cytochem 1985, 33 (8), 845.

(21) Zhang, L. F.; Ogawa, Y.; Ahn, J. Y.; Namekawa, S. H.; Silva, S. S.; Lee, J. T. Telomeric RNAs Mark Sex Chromosomes in Stem Cells. Genetics 2009, 182 (3), 685.

(22) Schoeftner, S.; Blasco, M. A. Developmentally regulated transcription of mammalian telomeres by DNA-dependent RNA polymerase II. Nat Cell Biol 2008, 10 (2), 228.

(23) Raj, A.; van den Bogaard, P.; Rifkin, S. A.; van Oudenaarden, A.; Tyagi, S. Imaging individual mRNA molecules using multiple singly labeled probes. Nat Methods 2008, 5 (10), 877.

(24) Wynes, M. W.; Hinz, T. K.; Gao, D. X.; Martini, M.; Marek, L. A.; Ware, K. E.; Edwards, M. G.; Bohm, D.; Perner, S.; Helfrich, B. A.et al. FGFR1 mRNA and Protein Expression, not Gene Copy Number, Predict FGFR TKI Sensitivity across All Lung Cancer Histologies. Clin Cancer Res 2014, 20 (12), 3299. 